BULLETIN (New Series) OF THE

AMERICAN MATHEMATICAL SOCIETY

Volume 47, Number 4, October 2010, Pages 567-638

S 0273-0979(2010)01304-5

Article electronically published on July 30, 2010

\title{
MATHEMATICAL GENERAL RELATIVITY: A SAMPLER
}

\author{
PIOTR T. CHRUŚCIEL, GREGORY J. GALLOWAY, AND DANIEL POLLACK
}

Abstract. We provide an introduction to selected recent advances in the mathematical understanding of Einstein's theory of gravitation.

\section{Contents}

1. Introduction 568

2. Elements of Lorentzian geometry and causal theory 5

2.1. Lorentzian manifolds $\quad 569$

2.2. Einstein equations 571

2.3. Elements of causal theory 571

2.3.1. Pasts and futures $\quad 572$

2.3.2. Causality conditions 573

2.3.3. Domains of dependence 575

2.4. Submanifolds 576

3. Stationary black holes

3.1. The Schwarzschild metric 577

3.2. Rotating black holes $\quad 579$

3.3. Killing horizons $\quad 580$

3.3.1. Surface gravity 581

3.3.2. Average surface gravity 5

3.4. Asymptotically flat metrics

3.5. Asymptotically flat stationary metrics

3.6. Domains of outer communications, event horizons

3.7. Uniqueness theorems 584

4. The Cauchy problem $\quad 586$

4.1. The local evolution problem 587

4.1.1. Wave-coordinates 587

4.2. Cauchy data 589

4.3. Solutions global in space 590

5. Initial data sets 592

5.1. The constraint equations 592

5.2. Mass inequalities 595

5.2.1. The Positive Mass Theorem 595

Received by the editors September 11, 2008 and, in revised form, November 6, 2008.

2010 Mathematics Subject Classification. Primary 83-02.

Support by the Banff International Research Station (Banff, Canada), and by Institut MittagLeffler (Djursholm, Sweden) is gratefully acknowledged. The research of the second author has been supported in part by an NSF grant DMS 0708048.

(C)2010 American Mathematical Society Reverts to public domain 28 years from publication 
5.2.2. Riemannian Penrose Inequality 596

5.2.3. Quasi-local mass 597

5.3. Applications of gluing techniques 597

5.3.1. The linearized constraint equations and KIDs 597

5.3.2. Corvino's result 5.58

5.3.3. Conformal gluing 599

5.3.4. Initial data engineering 600

6. Evolution 601

6.1. Strong cosmic censorship $\quad 601$

6.1.1. Gowdy toroidal metrics 603

6.1.2. Other $U(1) \times U(1)$-symmetric models 604

6.1.3. Spherical symmetry $\quad 604$

6.2. Weak cosmic censorship $\quad 605$

6.3. Stability of vacuum cosmological models 606

6.3.1. $U(1)$ symmetry 606

6.3.2. Future stability of hyperbolic models $\quad 607$

6.4. Stability of Minkowski space-time $\quad 607$

6.4.1. The Christodoulou-Klainerman proof $\quad 607$

6.4.2. The Lindblad-Rodnianski proof 608

6.5. Toward stability of Kerr: Wave equations on black hole backgrounds 610

6.6. Bianchi $A$ metrics $\quad 610$

6.7. The mixmaster conjecture 613

7. Marginally trapped surfaces $\quad 615$

7.1. Null hypersurfaces $\quad 615$

7.2. Trapped and marginally trapped surfaces $\quad 617$

7.3. Stability of MOTSs 620

7.4. On the topology of black holes $\quad 621$

7.5. Existence of MOTSs $\quad 623$

Appendix A. Open problems $\quad 625$

Acknowledgments $\quad 627$

About the authors $\quad 627$

References

\section{INTRODUCTION}

Mathematical general relativity is, by now, a well-established vibrant branch of mathematics. It ties fundamental problems of gravitational physics with beautiful questions in mathematics. The object is the study of manifolds equipped with a Lorentzian metric satisfying the Einstein field equations. Some highlights of its history include the discovery by Choquet-Bruhat of a well-posed Cauchy problem [140], subsequently globalized by Choquet-Bruhat and Geroch [75], the singularity theorems of Penrose and Hawking [151, 238], the proof of the positive mass theorem by Schoen and Yau [257, and the proof of stability of Minkowski space-time by Christodoulou and Klainerman [88.

There has recently been spectacular progress in the field on many fronts, including the Cauchy problem, stability, cosmic censorship, construction of initial data, 
and asymptotic behaviour, many of which will be described here. Mutual benefits are drawn, and progress is being made, from the interaction between general relativity and geometric analysis and the theory of elliptic and hyperbolic partial differential equations. The Einstein equation shares issues of convergence, collapse, and stability with other important geometric PDEs, such as the Ricci flow and the mean curvature flow. A steadily growing overlap between the relevant scientific communities can be seen. For all these reasons it appeared timely to provide a mathematically oriented reader with an introductory survey of the field. This is the purpose of the current work.

In Section 2 we survey the Lorentzian causality theory, the basic language for describing the structure of space-times. In Section 3 the reader is introduced to black holes, perhaps the most fascinating prediction of Einstein's theory of gravitation, and the source of many deep (solved or unsolved) mathematical problems. In Section 4 the Cauchy problem for the Einstein equations is considered, laying down the foundations for a systematic construction of general space-times. Section 5 examines initial data sets, as needed for the Cauchy problem, and their global properties. In Section 6 we discuss the dynamics of the Einstein equations, including questions of stability and predictability; the latter question is known under the baroque name of "strong cosmic censorship". Section 7 deals with trapped and marginally trapped surfaces, which signal the presence of black holes and have tantalizing connections with classical minimal surface theory. The paper is sprinkled with open problems, which are collected in Appendix $\mathrm{A}$

\section{Elements of Lorentzian GeOMEtry and CAUSAL THEORY}

2.1. Lorentzian manifolds. In general relativity, and related theories, the space of physical events is represented by a Lorentzian manifold. A Lorentzian manifold is a smooth (Hausdorff, paracompact) manifold $\mathscr{M}=\mathscr{M}^{n+1}$ of dimension $n+1$, equipped with a Lorentzian metric $g$. A Lorentzian metric is a smooth assignment to each point $p \in \mathscr{M}$ of a symmetric, nondegenerate bilinear form on the tangent space $T_{p} \mathscr{M}$ of signature $(-+\cdots+)$. Hence, if $\left\{e_{0}, e_{1}, \ldots, e_{n}\right\}$ is an orthonormal basis for $T_{p} \mathscr{M}$ with respect to $g$, then, perhaps after reordering the basis, the matrix $\left[g\left(e_{i}, e_{j}\right)\right]$ equals $\operatorname{diag}(-1,+1, \ldots,+1)$. A vector $v=\sum v^{\alpha} e_{\alpha}$ then has square norm,

$$
g(v, v)=-\left(v^{0}\right)^{2}+\sum\left(v^{i}\right)^{2},
$$

which can be positive, negative or zero. This leads to the causal character of vectors, and indeed to the causal theory of Lorentzian manifolds, which we shall discuss in Section 2.3 .

On a coordinate neighborhood $\left(U, x^{\alpha}\right)=\left(U, x^{0}, x^{1}, \ldots, x^{n}\right)$, the metric $g$ is completely determined by its metric component functions on $U, g_{\alpha \beta}:=g\left(\frac{\partial}{\partial x^{\alpha}}, \frac{\partial}{\partial x^{\beta}}\right)$, $0 \leq \alpha, \beta \leq n$ : For $v=v^{\alpha} \frac{\partial}{\partial x^{\alpha}}, w=w^{\beta} \frac{\partial}{\partial x^{\beta}} \in T_{p} \mathscr{M}, p \in U, g(v, w)=g_{\alpha \beta} v^{\alpha} w^{\beta}$. (Here we have used the Einstein summation convention: if, in a coordinate chart, an index appears repeated, once up and once down, then summation over that index is implied.) Classically the metric in coordinates is displayed via the line element, $d s^{2}=g_{\alpha \beta} d x^{\alpha} d x^{\beta}$.

The prototype Lorentzian manifold is Minkowski space $\mathbb{R}^{1, n}$, the space-time of special relativity. This is $\mathbb{R}^{n+1}$, equipped with the Minkowski metric, which, with respect to Cartesian coordinates $\left(x^{0}, x^{1}, \ldots, x^{n}\right)$, is given by

$$
d s^{2}=-\left(d x^{0}\right)^{2}+\left(d x^{1}\right)^{2}+\cdots+\left(d x^{n}\right)^{2} .
$$


Each tangent space of a Lorentzian manifold is isometric to Minkowski space, and in this way the local accuracy of special relativity is built into general relativity.

Every Lorentzian manifold (or, more generally, pseudo-Riemannian manifold) $\left(\mathscr{M}^{n+1}, g\right)$ comes equipped with a Levi-Civita connection (or covariant differentiation operator) $\nabla$ that enables one to compute the directional derivative of vector fields. Hence, for smooth vector fields $X, Y \in \mathfrak{X}(\mathscr{M}), \nabla_{X} Y \in \mathfrak{X}(\mathscr{M})$ denotes the covariant derivative of $Y$ in the direction $X$. The Levi-Civita connection is the unique connection $\nabla$ on $\left(\mathscr{M}^{n+1}, g\right)$ that is (i) symmetric (or torsion free), i.e., that satisfies $\nabla_{X} Y-\nabla_{Y} X=[X, Y]$ for all $X, Y \in \mathfrak{X}(M)$, and (ii) compatible with the metric, i.e. that obeys the metric product rule, $X(g(Y, Z))=g\left(\nabla_{X} Y, Z\right)+g\left(Y, \nabla_{X} Z\right)$, for all $X, Y, Z \in \mathfrak{X}(M)$.

In a coordinate chart $\left(U, x^{\alpha}\right)$ one has

$$
\nabla_{X} Y=\left(X\left(Y^{\mu}\right)+\Gamma_{\alpha \beta}^{\mu} X^{\alpha} Y^{\beta}\right) \partial_{\mu},
$$

where $X^{\alpha}, Y^{\alpha}$ are the components of $X$ and $Y$, respectively, with respect to the coordinate basis $\partial_{\alpha}=\frac{\partial}{\partial x^{\alpha}}$, and where the $\Gamma_{\alpha \beta}^{\mu}$ 's are the classical Christoffel symbols given in terms of the metric components by

$$
\Gamma_{\alpha \beta}^{\mu}=\frac{1}{2} g^{\mu \nu}\left(\partial_{\beta} g_{\alpha \nu}+\partial_{\alpha} g_{\beta \nu}-\partial_{\nu} g_{\alpha \beta}\right) \text {. }
$$

Note that the coordinate expression (2.2) can also be written as

$$
\nabla_{X} Y=X^{\alpha} \nabla_{\alpha} Y^{\mu} \partial_{\mu}
$$

where $\nabla_{\alpha} Y^{\mu}$ (often written classically as $Y^{\mu} ; \alpha$ ) is given by

$$
\nabla_{\alpha} Y^{\mu}=\partial_{\alpha} Y^{\mu}+\Gamma_{\alpha \beta}^{\mu} Y^{\beta}
$$

We shall feel free to interchange between coordinate and coordinate-free notation. The Levi-Civita connection $\nabla$ extends in a natural way to a covariant differentiation operator on all tensor fields.

The Riemann curvature tensor of $\left(\mathscr{M}^{n+1}, g\right)$ is the map $R: \mathfrak{X}(M) \times \mathfrak{X}(M) \times$ $\mathfrak{X}(M) \rightarrow \mathfrak{X}(M),(X, Y, Z) \rightarrow R(X, Y) Z$, given by

$$
R(X, Y) Z=\nabla_{X} \nabla_{Y} Z-\nabla_{Y} \nabla_{X} Z-\nabla_{[X, Y]} Z \text {. }
$$

This expression is linear in $X, Y, Z \in \mathfrak{X}(\mathscr{M})$ with respect to $C^{\infty}(\mathscr{M})$. This implies that $R$ is indeed tensorial, i.e., that the value of $R(X, Y) Z$ at $p \in M$ depends only on the value of $X, Y, Z$ at $p$.

Equation (2.6) shows that the Riemann curvature tensor measures the extent to which covariant differentiation fails to commute. This failure to commute may be seen as an obstruction to the existence of parallel vector fields. By Riemann's theorem, a Lorentzian manifold is locally Minkowskian if and only if the Riemann curvature tensor vanishes.

The components $R^{\mu}{ }_{\gamma \beta}$ of the Riemann curvature tensor $R$ in a coordinate chart $\left(U, x^{\alpha}\right)$ are determined by the equations $R\left(\partial_{\alpha}, \partial_{\beta}\right) \partial_{\gamma}=R_{\gamma \alpha \beta}^{\mu} \partial_{\mu}$. Equations (2.2) and $(2.6)$ then yield the following explicit formula for the curvature components in terms of the Christoffel symbols:

$$
R_{\gamma \alpha \beta}^{\mu}=\partial_{\alpha} \Gamma_{\gamma \beta}^{\mu}-\partial_{\beta} \Gamma_{\gamma \alpha}^{\mu}+\Gamma_{\gamma \beta}^{\nu} \Gamma_{\nu \alpha}^{\mu}-\Gamma_{\gamma \alpha}^{\nu} \Gamma_{\nu \beta}^{\mu} .
$$

The Ricci tensor, Ric, is a bilinear form obtained by contraction of the Riemann curvature tensor, i.e., its components $R_{\mu \nu}=\operatorname{Ric}\left(\partial_{\mu}, \partial_{\nu}\right)$ are determined by tracing, $R_{\mu \nu}=R_{\mu \alpha \nu}^{\alpha}$. Symmetries of the Riemann curvature tensor imply that the Ricci 
tensor is symmetric, $R_{\mu \nu}=R_{\nu \mu}$. By tracing the Ricci tensor, we obtain the scalar curvature $R=g^{\mu \nu} R_{\mu \nu}$, where $g^{\mu \nu}$ denotes the matrix inverse to $g_{\mu \nu}$.

2.2. Einstein equations. The Einstein equation (with cosmological constant $\Lambda$ ), the field equation of general relativity, is the tensor equation

$$
\text { Ric }-\frac{1}{2} R g+\Lambda g=8 \pi \mathcal{T},
$$

where $\mathcal{T}$ is the energy-momentum tensor (see, e.g., Section 6.4.2 for an example of an energy-momentum tensor). When expressed in terms of coordinates, the Einstein equation becomes a system of second-order equations for the metric components $g_{\mu \nu}$ and the nongravitational field variables introduced through the energy-momentum tensor. We say that space-time obeys the vacuum Einstein equation if it obeys the Einstein equation with $\mathcal{T}=0$.

The Riemann curvature tensor has a number of symmetry properties, one of which is the so-called first Bianchi identity:

$$
R_{\alpha \beta \gamma \delta}+R_{\alpha \gamma \delta \beta}+R_{\alpha \delta \beta \gamma}=0 .
$$

The curvature tensor also obeys a differential identity known as the second Bianchi identity:

$$
\nabla_{\sigma} R_{\alpha \beta \gamma \delta}+\nabla_{\alpha} R_{\beta \sigma \gamma \delta}+\nabla_{\beta} R_{\sigma \alpha \gamma \delta}=0 .
$$

When twice contracted, (2.9) yields the following divergence identity:

$$
\nabla_{\alpha}\left(R^{\alpha \beta}-\frac{R}{2} g^{\alpha \beta}\right)=0 .
$$

This plays a fundamental role in general relativity, as, in particular, it implies, in conjunction with the Einstein equation, local conservation of energy, $\nabla_{\alpha} T^{\alpha \beta}=0$. It also plays an important role in the mathematical analysis of the Einstein equations; see Section 4 for further discussion.

2.3. Elements of causal theory. Many concepts and results in general relativity make use of the causal theory of Lorentzian manifolds. The starting point for causal theory is the causal classification of tangent vectors. Let $\left(\mathscr{M}^{n+1}, g\right)$ be a Lorentzian manifold. A vector $v \in T_{p} \mathscr{M}$ is timelike (resp., spacelike, null) provided $g(v, v)<0$ (resp., $g(v, v)>0, g(v, v)=0$ ). The collection of null vectors forms a double cone $\mathcal{V}_{p}$ in $T_{p} \mathscr{M}$ (recall (2.1)), called the null cone at $p$; see Figure 2.1

The timelike vectors at $p$ point inside the null cone and the spacelike vectors point outside. We say that $v \in T_{p} \mathscr{M}$ is causal if it is timelike or null. We define the length of causal vectors as $|v|=\sqrt{-g(v, v)}$. Causal vectors $v, w \in T_{p} \mathscr{M}$ that point into the same half-cone of the null cone $\mathcal{V}_{p}$ obey the reverse triangle inequality, $|v+w| \geq|v|+|w|$. Geometrically, this is the source of the twin paradox.

These notions of causality extend to curves. Let $\gamma: I \rightarrow \mathscr{M}, t \rightarrow \gamma(t)$, be a smooth curve in $\mathscr{M}$, then $\gamma$ is said to be timelike (resp., spacelike, null, causal) provided each of its velocity vectors $\gamma^{\prime}(t)$ is timelike (resp., spacelike, null, causal). Heuristically, in accordance with relativity, information flows along causal curves, and so such curves are the focus of attention in causal theory. The notion of a causal curve extends in a natural way to piecewise smooth curves, and we will normally work within this class. As usual, we define a geodesic to be a curve $t \rightarrow \gamma(t)$ of zero covariant acceleration, $\nabla_{\gamma^{\prime}} \gamma^{\prime}=0$. Since geodesics $\gamma$ are constant 


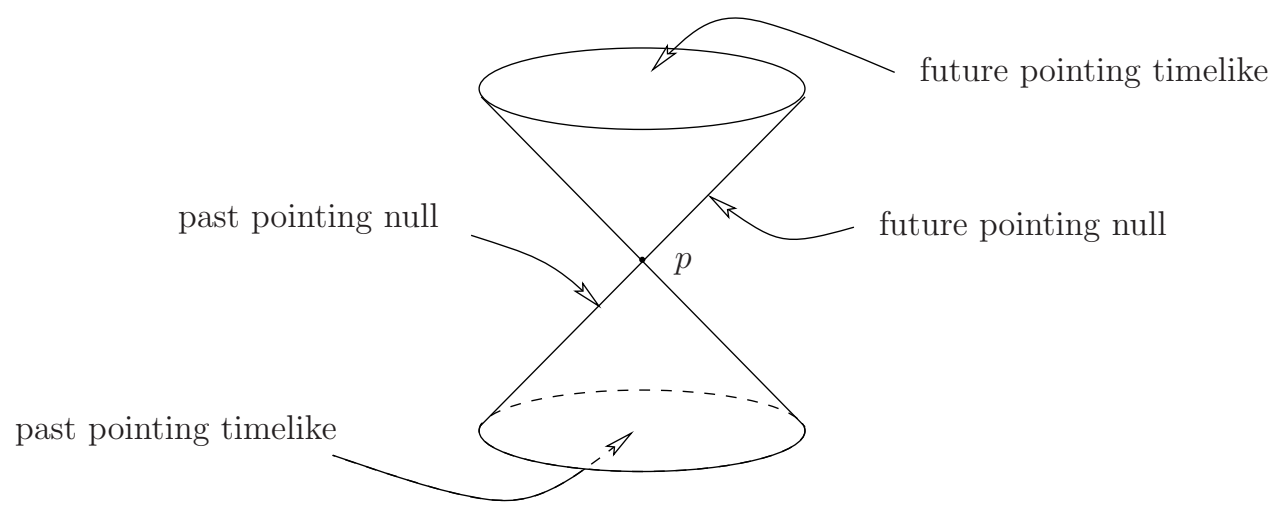

Figure 2.1. The light cone at $p$.

speed curves $\left(g\left(\gamma^{\prime}, \gamma^{\prime}\right)=\right.$ const. $)$, each geodesic in a Lorentzian manifold is either timelike, spacelike or null.

The length of a causal curve $\gamma:[a, b] \rightarrow \mathscr{M}$ is defined as

$$
L(\gamma)=\text { Length of } \gamma=\int_{a}^{b}\left|\gamma^{\prime}(t)\right| d t=\int_{a}^{b} \sqrt{-g\left(\gamma^{\prime}(t), \gamma^{\prime}(t)\right)} d t .
$$

If $\gamma$ is timelike, one can introduce an arc length parameter along $\gamma$. In general relativity, a timelike curve corresponds to the history of an observer, and the arc length parameter, called proper time, corresponds to time kept by the observer. Using the existence and properties of geodesically convex neighborhoods 232 one can show that causal geodesics are locally maximal (i.e., locally longest among causal curves).

Each null cone $\mathcal{V}_{p}$ consists of two half-cones, one of which may designated as the future cone and the other as the past cone at $p$. If the assignment of a past and future cone at each point of $\mathscr{M}$ can be carried out in a continuous manner over $\mathscr{M}$, then $\mathscr{M}$ is said to be time-orientable. There are various ways to make the phrase "continuous assignment" precise, but they all result in the following fact: a Lorentzian manifold $\left(\mathscr{M}^{n+1}, g\right)$ is time-orientable if and only if it admits a smooth timelike vector field $Z$. If $\mathscr{M}$ is time-orientable, the choice of a smooth timelike vector field $Z$ fixes a time orientation on $\mathscr{M}$ : For any $p \in \mathscr{M}$, a causal vector $v \in T_{p} \mathscr{M}$ is future directed (resp. past directed) provided $g(v, Z)<0$ (resp. $g(v, Z)>0)$. Thus, $v$ is future directed if it points into the same null half-cone at $p$ as $Z$. We note that a Lorentzian manifold that is not time-orientable always admits a double cover that is. By a space-time we mean a connected time-oriented Lorentzian manifold $\left(\mathscr{M}^{n+1}, g\right)$. Henceforth, we restrict our attention to spacetimes.

2.3.1. Pasts and futures. Let $(\mathscr{M}, g)$ be a space-time. A timelike (resp. causal) curve $\gamma: I \rightarrow \mathscr{M}$ is said to be future directed provided each tangent vector $\gamma^{\prime}(t)$, $t \in I$, is future directed. (Past-directed timelike and causal curves are defined in a time-dual manner.) $I^{+}(p)$, the timelike future of $p \in \mathscr{M}$, is the set consisting of all points $q \in \mathscr{M}$ for which there exists a future-directed timelike curve from $p$ to q. $J^{+}(p)$, the causal future of $p \in \mathscr{M}$, is the set consisting of $p$ and all points $q$ for which there exists a future-directed causal curve from $p$ to $q$. In Minkowski space 
$\mathbb{R}^{1, n}$ these sets have a simple structure: For each $p \in \mathbb{R}^{1, n}, \partial I^{+}(p)=J^{+}(p) \backslash I^{+}(p)$ is the future cone at $p$ generated by the future-directed null rays emanating from p. $I^{+}(p)$ consists of the points inside the cone, and $J^{+}(p)$ consists of the points on and inside the cone. In general, the curvature and topology of space-time can strongly influence the structure of these sets.

Since a timelike curve remains timelike under small smooth perturbations, it is heuristically clear that the sets $I^{+}(p)$ are in general open; a careful proof makes use of properties of geodesically convex sets. On the other hand, the sets $J^{+}(p)$ need not be closed in general, as can be seen by considering the space-time obtained by removing a point from Minkowski space.

It follows from variational arguments that, for example, if $q \in I^{+}(p)$ and $r \in$ $J^{+}(q)$, then $r \in I^{+}(p)$. This and related claims are in fact a consequence of the following fundamental causality result [232].

Proposition 2.1. If $q \in J^{+}(p) \backslash I^{+}(p)$, i.e., if $q$ is in the causal future of $p$ but not in the timelike future of $p$, then any future-directed causal curve from $p$ to $q$ must be a null geodesic.

Given a subset $S \subset \mathscr{M}, I^{+}(S)$, the timelike future of $S$, consists of all points $q \in \mathscr{M}$ for which there exists a future-directed timelike curve from a point in $S$ to q. $J^{+}(S)$, the causal future of $S$, consists of the points of $S$ and all points $q \in \mathscr{M}$ for which there exists a future-directed causal curve from a point in $S$ to $q$. Note that $I^{+}(S)=\bigcup_{p \in S} I^{+}(p)$. Hence, as a union of open sets, $I^{+}(S)$ is always open.

The timelike and causal pasts $I^{-}(p), J^{-}(p), I^{-}(S), J^{-}(S)$ are defined in a timedual manner in terms of past-directed timelike and causal curves. It is sometimes convenient to consider pasts and futures within some open subset $U$ of $\mathscr{M}$. For example, $I^{+}(p, U)$ denotes the set consisting of all points $q \in U$ for which there exists a future-directed timelike curve from $p$ to $q$ contained in $U$.

Sets of the form $\partial I^{ \pm}(S)$ are called achronal boundaries and have nice structural properties: they are achronal Lipschitz hypersurfaces, ruled, in a certain sense, by null geodesics 232. (A set is achronal if no two of its points can be joined by a timelike curve.)

2.3.2. Causality conditions. A number of results in Lorentzian geometry and general relativity require some sort of causality condition. It is perhaps natural on physical grounds to rule out the occurrence of closed timelike curves. Physically, the existence of such a curve signifies the existence of an observer who is able to travel into his/her own past, which leads to a variety of paradoxical situations. A space-time $\mathscr{M}$ satisfies the chronology condition provided there are no closed timelike curves in $\mathscr{M}$. It can be shown that all compact space-times violate the chronology condition, and for this reason compact space-times have been of limited interest in general relativity.

A somewhat stronger condition than the chronology condition is the causality condition. A space-time $\mathscr{M}$ satisfies the causality condition provided there are no closed (nontrivial) causal curves in $\mathscr{M}$. A slight weakness of this condition is that there are space-times which satisfy the causality condition, but contain causal curves that are almost closed; see, e.g., [154, p. 193].

It is useful to have a condition that rules out almost closed causal curves. A space-time $\mathscr{M}$ is said to be strongly causal at $p \in \mathscr{M}$ provided there are arbitrarily small neighborhoods $U$ of $p$ such that any causal curve $\gamma$ which starts in and leaves 
$U$ never returns to $U$. $\mathscr{M}$ is strongly causal if it is strongly causal at each of its points. Thus, heuristically speaking, $\mathscr{M}$ is strongly causal provided there are no closed or almost closed causal curves in $\mathscr{M}$. Strong causality is the standard causality condition of space-time geometry, and although there are even stronger causality conditions, it is sufficient for most applications. A very useful fact about strongly causal space-times is the following: If $\mathscr{M}$ is strongly causal, then any future (or past) inextendible causal curve $\gamma$ cannot be imprisoned or partially imprisoned in a compact set. That is to say, if $\gamma$ starts in a compact set $K$, it must eventually leave $K$ for good.

We now come to a fundamental condition in space-time geometry, that of global hyperbolicity. Mathematically, global hyperbolicity is a basic niceness condition that often plays a role analogous to geodesic completeness in Riemannian geometry. Physically, global hyperbolicity is connected to the notion of strong cosmic censorship, the conjecture that, generically, space-time solutions to the Einstein equations do not admit naked (i.e., observable) singularities; see Section 6.1 for further discussion.

A space-time $\mathscr{M}$ is said to be globally hyperbolic provided:

(1) $\mathscr{M}$ is strongly causal.

(2) (Internal Compactness) The sets $J^{+}(p) \cap J^{-}(q)$ are compact for all $p, q \in \mathscr{M}$.

Condition (2) says roughly that $\mathscr{M}$ has no holes or gaps. For example, Minkowski space $\mathbb{R}^{1, n}$ is globally hyperbolic but the space-time obtained by removing one point from it is not. Leray [194] was the first to introduce the notion of global hyperbolicity (in a somewhat different but equivalent form) in connection with his study of the Cauchy problem for hyperbolic PDEs.

We mention a couple of basic consequences of global hyperbolicity. Firstly, globally hyperbolic space-times are causally simple, by which is meant that the sets $J^{ \pm}(A)$ are closed for all compact $A \subset \mathscr{M}$. This fact and internal compactness imply that the sets $J^{+}(A) \cap J^{-}(B)$ are compact for all compact $A, B \subset \mathscr{M}$.

Analogously to the case of Riemannian geometry, one can learn much about the global structure of space-time by studying its causal geodesics. Global hyperbolicity is the standard condition in Lorentzian geometry that guarantees the existence of maximal timelike geodesic segments joining timelike related points. More precisely, one has the following.

Proposition 2.2. If $\mathscr{M}$ is globally hyperbolic and $q \in I^{+}(p)$, then there exists a maximal timelike geodesic segment $\gamma$ from $p$ to $q$, where by maximal, we mean $L(\gamma) \geq L(\sigma)$ for all future-directed causal curves $\sigma$ from $p$ to $q$.

Contrary to the situation in Riemannian geometry, geodesic completeness does not guarantee the existence of maximal segments, as is well illustrated by anti-de Sitter space; see, e.g., [27.

Global hyperbolicity is closely related to the existence of certain ideal initial value hypersurfaces, called Cauchy (hyper)surfaces. There are slight variations in the literature in the definition of a Cauchy surface. Here we adopt the following definition: A Cauchy surface for a space-time $\mathscr{M}$ is a subset $S$ that is met exactly once by every inextendible causal curve in $\mathscr{M}$. It can be shown that a Cauchy surface for $\mathscr{M}$ is necessarily a $C^{0}$ (in fact, Lipschitz) hypersurface in $\mathscr{M}$. Note also that a Cauchy surface is acausal, that is, no two of its points can be joined by a causal curve. The following result is fundamental. 
Proposition 2.3 (Geroch [146]). $\mathscr{M}$ is globally hyperbolic if and only if $\mathscr{M}$ admits a Cauchy surface. If $S$ is a Cauchy surface for $\mathscr{M}$, then $\mathscr{M}$ is homeomorphic to $\mathbb{R} \times S$.

With regard to the implication that global hyperbolicity implies the existence of a Cauchy surface, Geroch, in fact, proved something substantially stronger. (We will make some comments about the converse in Section 2.3.3) A time function on $\mathscr{M}$ is a $C^{0}$ function $t$ on $\mathscr{M}$ such that $t$ is strictly increasing along every futuredirected causal curve. Geroch established the existence of a time function $t$, all of whose level sets $t=t_{0}, t_{0} \in \mathbb{R}$, are Cauchy surfaces. This result can be strengthened to the smooth category. By a smooth time function we mean a smooth function $t$ with everywhere past-pointing timelike gradient. This implies that $t$ is strictly increasing along all future directed causal curves, and that its level sets are smooth spacelike1 hypersurfaces. It has been shown that a globally hyperbolic space-time admits a smooth time function, all of whose levels sets are Cauchy surfaces 42, 265. In fact, one obtains a diffeomorphism $\mathscr{M} \approx \mathbb{R} \times S$, where the $\mathbb{R}$-factor corresponds to a smooth time function, such that each slice $S_{t}=\{t\} \times S, t \in \mathbb{R}$, is a Cauchy surface.

Given a Cauchy surface $S$ to begin with, to simply show that $\mathscr{M}$ is homeomorphic to $\mathbb{R} \times S$, consider a complete timelike vector field $Z$ on $\mathscr{M}$ and observe that each integral curve of $Z$, when maximally extended, meets $S$ in a unique point. This leads to the desired homeomorphism. (If $S$ is smooth, this will be a diffeomorphism.) In a similar vein, one can show that any two Cauchy surfaces are homeomorphic. Thus, the topology of a globally hyperbolic space-time is completely determined by the common topology of its Cauchy surfaces.

The following result is often useful.

Proposition 2.4. Let $\mathscr{M}$ be a space-time.

(1) If $S$ is a compact acausal $C^{0}$ hypersurface and $\mathscr{M}$ is globally hyperbolic, then $S$ must be a Cauchy surface for $\mathscr{M}$.

(2) If $t$ is a smooth time function on $\mathscr{M}$ all of whose level sets are compact, then each level set is a Cauchy surface for $\mathscr{M}$, and hence $\mathscr{M}$ is globally hyperbolic.

We will comment on the proof shortly, after Proposition 2.6.

2.3.3. Domains of dependence. The future domain of dependence of an acausal set $S$ is the set $\mathscr{D}^{+}(S)$ consisting of all points $p \in \mathscr{M}$ such that every past inextendible causal curv 2 from $p$ meets $S$. In physical terms, since information travels along causal curves, a point in $\mathscr{D}^{+}(S)$ only receives information from $S$. Thus, in principle, $\mathscr{D}^{+}(S)$ represents the region of space-time to the future of $S$ that is predictable from $S$. $\mathscr{H}^{+}(S)$, the future Cauchy horizon of $S$, is defined to be the future boundary of $\mathscr{D}^{+}(S)$; in precise terms, $\mathscr{H}^{+}(S)=\left\{p \in \overline{\mathscr{D}^{+}(S)}: I^{+}(p) \cap \mathscr{D}^{+}(S)=\emptyset\right\}$. Physically, $\mathscr{H}^{+}(S)$ is the future limit of the region of space-time predictable from $S$. Some examples of domains of dependence, and Cauchy horizons, can be found in Figure 2.2.

\footnotetext{
${ }^{1}$ A hypersurface is called spacelike if the induced metric is Riemannian; see Section 2.4

${ }^{2}$ We note that some authors use past inextendible timelike curves to define the future domain of dependence, which results in some small differences in certain results.
} 

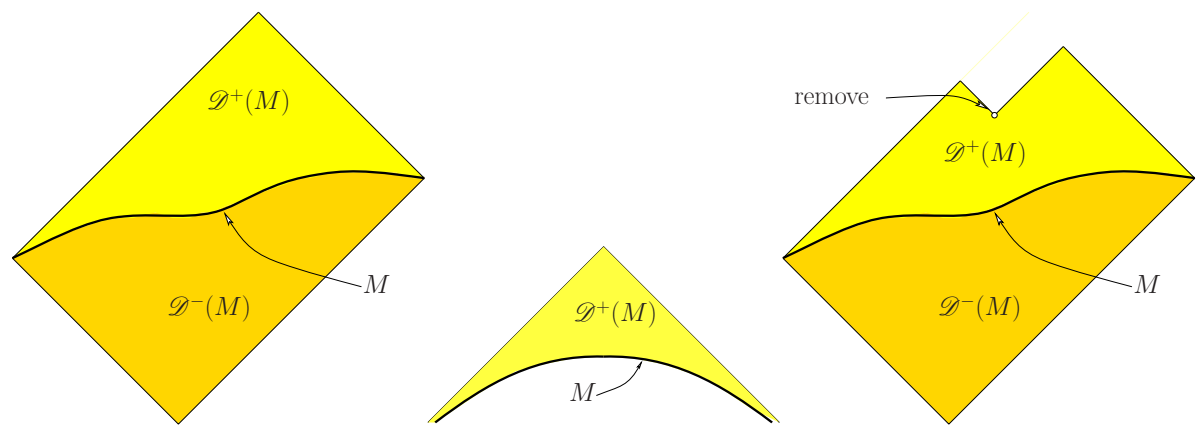

Figure 2.2. Examples of domains of dependence and Cauchy horizons.

It follows almost immediately from the definition that $\mathscr{H}^{+}(S)$ is achronal. In fact, Cauchy horizons have structural properties similar to achronal boundaries, as indicated in the following.

Proposition 2.5. Let $S$ be an acausal subset of a space-time $\mathscr{M}$. Then $\mathscr{H}^{+}(S) \backslash \bar{S}$, if nonempty, is an achronal $C^{0}$ hypersurface of $\mathscr{M}$ ruled by null geodesics, called generators, each of which is either past inextendible in $\mathscr{M}$ or has past endpoint on $\bar{S}$.

The past domain of dependence $\mathscr{D}^{-}(S)$ of $S$ and the past Cauchy horizon $\mathscr{H}^{-}(S)$ of $S$ are defined in a time-dual manner. The total domain of dependence $\mathscr{D}(S)$ and the total Cauchy horizon $\mathscr{H}(S)$ are defined, respectively, as $\mathscr{D}(S)=\mathscr{D}^{+}(S) \cup \mathscr{D}^{-}(S)$ and $\mathscr{H}(S)=\mathscr{H}^{+}(S) \cup \mathscr{H}^{-}(S)$.

Domains of dependence may be used to characterize Cauchy surfaces. In fact, it follows easily from the definitions that an acausal subset $S \subset \mathscr{M}$ is a Cauchy surface for $\mathscr{M}$ if and only if $\mathscr{D}(S)=\mathscr{M}$. Using the fact that $\partial \mathscr{D}(S)=\mathscr{H}(S)$, we obtain the following.

Proposition 2.6. Let $S$ be an acausal subset of a space-time $\mathscr{M}$. Then, $S$ is a Cauchy surface for $\mathscr{M}$ if and only if $\mathscr{D}(S)=\mathscr{M}$ if and only if $\mathscr{H}(S)=\emptyset$.

Part 1 of Proposition 2.4 can now be readily proved by showing, with the aid of Proposition 2.5. that $\mathscr{H}(S)=\emptyset$. Indeed if $\mathscr{H}^{+}(S) \neq \emptyset$, then there exists a past inextendible null geodesic $\eta \subset \mathscr{H}^{+}(S)$ with future endpoint $p$ imprisoned in the compact set $J^{+}(S) \cap J^{-}(p)$ which, as already mentioned, is not possible in strongly causal space-times. Part 2 is proved similarly; compare [60, 143].

The following basic result ties domains of dependence to global hyperbolicity.

Proposition 2.7. Let $S \subset \mathscr{M}$ be acausal.

(1) Strong causality holds at each point of int $\mathscr{D}(S)$.

(2) Internal compactness holds on int $\mathscr{D}(S)$; i.e., for all $p, q \in \operatorname{int} \mathscr{D}(S), J^{+}(p) \cap$ $J^{-}(q)$ is compact.

Propositions 2.6 and 2.7 immediately imply that if $S$ is a Cauchy surface for a space-time $\mathscr{M}$, then $\mathscr{M}$ is globally hyperbolic, as claimed in Proposition 2.3 .

2.4. Submanifolds. In addition to curves, one may also speak of the causal character of higher-dimensional submanifolds. Let $V$ be a smooth submanifold of a 
space-time $(\mathscr{M}, g)$. For $p \in V$, we say that the tangent space $T_{p} V$ is spacelike (resp. timelike, null) provided $g$ restricted to $T_{p} V$ is positive definite (resp., has Lorentzian signature, is degenerate). Then $V$ is said to be spacelike (resp., timelike, null) provided each of its tangent spaces is spacelike (resp., timelike, null). Hence, if $V$ is spacelike (resp., timelike), then, with respect to its induced metric (i.e., the metric $g$ restricted to the tangent spaces of $V$ ), $V$ is a Riemannian (resp., Lorentzian) manifold.

\section{Stationary BlaCK HOLES}

Perhaps the first thing which comes to mind when general relativity is mentioned are black holes. These are among the most fascinating objects predicted by Einstein's theory of gravitation. In this section we focus attention on stationary black holes that are solutions of the vacuum Einstein equations with vanishing cosmological constant, with one exception: the static electro-vacuum Majumdar-Papapetrou solutions, an example of physically significant multiple black holes. By definition, a stationary space-time is an asymptotically flat space-time which is invariant under an action of $\mathbb{R}$ by isometries, such that the associated generator - referred to as the Killing vector - is timelike in the asymptotically flat region. These model steady-state solutions. Stationary black holes are the simplest to describe, and most mathematical results on black holes, such as the uniqueness theorems discussed in Section 3.7 concern those. It should, however, be kept in mind that one of the major open problems in mathematical relativity is the understanding of the dynamical behavior of black hole space-times, about which not much is yet known (compare Section 6.5).

3.1. The Schwarzschild metric. The simplest stationary solutions describing compact isolated objects are the spherically symmetric ones. According to Birkhoff's theorem [45, any $(n+1)$-dimensional, $n \geq 3$, spherically symmetric solution of the vacuum Einstein equations belongs to the family of Schwarzschild metrics, parameterized by a mass parameter $m$ :

$$
\begin{gathered}
g=-V^{2} d t^{2}+V^{-2} d r^{2}+r^{2} d \Omega^{2}, \\
V^{2}=1-\frac{2 m}{r^{n-2}}, \quad t \in \mathbb{R}, r \in(2 m, \infty) .
\end{gathered}
$$

Here $d \Omega^{2}$ denotes the metric of the standard $(n-1)$-sphere. (This is true without assuming stationarity.)

From now on we assume $n=3$, though identical results hold in higher dimensions.

We will assume

$$
m>0
$$

because $m<0$ leads to metrics which are called nakedly singular; this deserves a comment. For Schwarzschild metrics we have

$$
R_{\alpha \beta \gamma \delta} R^{\alpha \beta \gamma \delta}=\frac{48 m^{2}}{r^{6}}
$$

in dimension $3+1$, which shows that the geometry becomes singular as $r=0$ is approached; this remains true in higher dimensions. As we shall see shortly, for $m>0$ the singularity is hidden behind an event horizon, while this is not the case for $m<0$. 
One of the first features one notices is that the metric (3.1) is singular as $r=2 \mathrm{~m}$ is approached. It turns out that this singularity is related to an unfortunate choice of coordinates (one talks about a coordinate singularity); the simplest way to see this is to replace $t$ by a new coordinate $v$ defined as

$$
v=t+f(r), \quad f^{\prime}=\frac{1}{V^{2}},
$$

leading to

$$
v=t+r+2 m \ln (r-2 m) .
$$

This brings $g$ to the form

$$
g=-\left(1-\frac{2 m}{r}\right) d v^{2}+2 d v d r+r^{2} d \Omega^{2} .
$$

We have $\operatorname{det} g=-r^{4} \sin ^{2} \theta$, with all coefficients of $g$ smooth, which shows that $g$ is a well-defined Lorentzian metric on the set

$$
v \in \mathbb{R}, \quad r \in(0, \infty) .
$$

More precisely, (3.5)-(3.6) provides an analytic extension of the original space-time (3.1).

It is easily seen that the region $\{r \leq 2 m\}$ for the metric (3.5) is a black hole region, in the sense that

observers, or signals, can enter this region, but can never leave it.

In order to see that, recall that observers in general relativity always move on future-directed timelike curves, that is, curves with a timelike future-directed tangent vector. For signals, the curves are causal future directed. Let, then, $\gamma(s)=$ $(v(s), r(s), \theta(s), \varphi(s))$ be such a timelike curve; for the metric (3.5) the timelikeness condition $g(\dot{\gamma}, \dot{\gamma})<0$ reads

$$
-\left(1-\frac{2 m}{r}\right) \dot{v}^{2}+2 \dot{v} \dot{r}+r^{2}\left(\dot{\theta}^{2}+\sin ^{2} \theta \dot{\varphi}^{2}\right)<0 .
$$

This implies that

$$
\dot{v}\left(-\left(1-\frac{2 m}{r}\right) \dot{v}+2 \dot{r}\right)<0 .
$$

It follows that $\dot{v}$ does not change sign on a timelike curve. The usual choice of time orientation corresponds to $\dot{v}>0$ on future-directed curves, leading to

$$
-\left(1-\frac{2 m}{r}\right) \dot{v}+2 \dot{r}<0 \text {. }
$$

For $r \leq 2 m$ the first term is nonnegative, which enforces $\dot{r}<0$ on all futuredirected timelike curves in that region. Thus, $r$ is a strictly decreasing function along such curves, which implies that future-directed timelike curves can cross the hypersurface $\{r=2 m\}$ only if coming from the region $\{r>2 m\}$. This motivates the name black hole event horizon for $\{r=2 m, v \in \mathbb{R}\}$. The same conclusion (3.7) applies for causal curves: it suffices to approximate a causal curve by a sequence of timelike ones.

The transition from (3.1) to (3.5) is not the end of the story, as further extensions are possible. For the metric (3.1) a maximal analytic extension has been found independently by Kruskal [192], Szekeres [270], and Fronsdal [142]; for some obscure reason Fronsdal is almost never mentioned in this context. This extension 
is depicted 3 in Figure 3.1. The region $I$ there corresponds to the space-time (3.1), while the extension just constructed corresponds to the regions $I$ and $I I$.

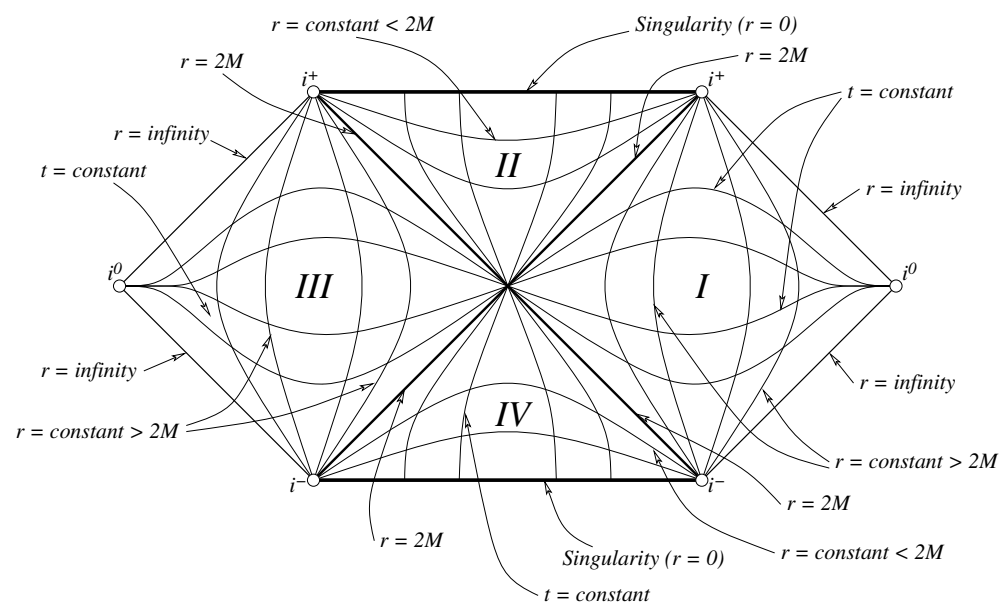

Figure 3.1. The Carter-Penrose diagram ${ }^{3}$ for the KruskalSzekeres space-time with mass $M$. There are actually two asymptotically flat regions, with corresponding event horizons defined with respect to the second region. Each point in this diagram represents a two-dimensional sphere, and coordinates are chosen so that light-cones have slopes plus minus one.

The Kruskal-Szekeres extension is singled out by being maximal in the class of vacuum, analytic, simply connected space-times, with all maximally extended geodesics $\gamma$ either complete or with the curvature scalar $R_{\alpha \beta \gamma \delta} R^{\alpha \beta \gamma \delta}$ diverging along $\gamma$ in finite affine time.

An alternative convenient representation of the Schwarzschild metrics, which makes the space-part of $g$ manifestly conformally flat, is given by

$$
g=-\left(\frac{1-m / 2|x|^{n-2}}{1+m / 2|x|^{n-2}}\right)^{2} d t^{2}+\left(1+\frac{m}{2|x|^{n-2}}\right)^{\frac{4}{n-2}}\left(\sum_{1=1}^{n}\left(d x^{i}\right)^{2}\right) .
$$

3.2. Rotating black holes. Rotating generalizations of the Schwarzschild metrics are given by the family of Kerr metrics, parameterized by a mass parameter $m$ and an angular momentum parameter $a$. One explicit coordinate representation of the Kerr metric is

$$
\begin{aligned}
\hat{g}= & -\left(1-\frac{2 m r}{\Sigma}\right) d v^{2}+2 d r d v+\Sigma d \theta^{2}-2 a \sin ^{2} \theta d \phi d r \\
& +\frac{\left(r^{2}+a^{2}\right)^{2}-a^{2} \Delta \sin ^{2} \theta}{\Sigma} \sin ^{2} \theta d \phi^{2}-\frac{4 a m r \sin ^{2} \theta}{\Sigma} d \phi d v,
\end{aligned}
$$

where

$$
\Sigma=r^{2}+a^{2} \cos ^{2} \theta, \quad \Delta=r^{2}+a^{2}-2 m r .
$$

\footnotetext{
${ }^{3}$ We are grateful to J.-P. Nicolas for allowing us to use his figure from 229 .
} 
Note that (3.9) reduces to the Schwarzschild solution in the representation (3.5) when $a=0$. The reader is referred to [65, 233] for a thorough analysis. All Kerr metrics satisfying

$$
m^{2} \geq a^{2}
$$

provide, when appropriately extended, vacuum space-times containing a rotating black hole. Higher-dimensional analogues of the Kerr metrics have been constructed by Myers and Perry 225.

A fascinating class of black hole solutions of the $(4+1)$-dimensional stationary vacuum Einstein equations has been found by Emparan and Reall [133] (see also 97, 98, 132, 134, 135, 241). The solutions, called black rings, are asymptotically Minkowskian in spacelike directions, with an event horizon having $S^{1} \times S^{2}$ cross-sections. The "ring" terminology refers to the $S^{1}$ factor in $S^{1} \times S^{2}$.

3.3. Killing horizons. Before continuing, some general notions are in order. By definition, a Killing field is a vector field, the local flow of which preserves the metric. Killing vectors are solutions of the over-determined system of Killing equations

$$
\nabla_{\alpha} X_{\beta}+\nabla_{\beta} X_{\alpha}=0 \text {. }
$$

One of the features of the metric (3.1) is its stationarity, with Killing vector field $X=\partial_{t}$ : As already pointed out, a space-time is called stationary if there exists a Killing vector field $X$ which approaches $\partial_{t}$ in the asymptotically flat region (where $r$ goes to $\infty$; see Section 3.4 for precise definitions) and generates a one-parameter group of isometries. A space-time is called static if it is stationary and if the distribution of hyperplanes orthogonal to the stationary Killing vector $X$ is integrable.

A space-time is called axisymmetric if there exists a Killing vector field $Y$ which generates a one-parameter group of isometries and which behaves like a rotation: this property is captured by requiring that all orbits be $2 \pi$-periodic, and that the set $\{Y=0\}$, called the axis of rotation, be nonempty.

Let $X$ be a Killing vector field on $(\mathscr{M}, g)$, and suppose that $\mathscr{M}$ contains a null hypersurface (see Sections 2.4 and 7.1) $\mathscr{N}_{0}=\mathscr{N}_{0}(X)$ which coincides with a connected component of the set

$$
\mathscr{N}(X):=\left\{p \in \mathscr{M} \mid g\left(X_{p}, X_{p}\right)=0, X_{p} \neq 0\right\},
$$

with $X$ tangent to $\mathscr{N}_{0}$. Then $\mathscr{N}_{0}$ is called a Killing horizon associated to the Killing vector $X$. The simplest example is provided by the boost Killing vector field

$$
K=z \partial_{t}+t \partial_{z}
$$

in four-dimensional Minkowski space-time $\mathbb{R}^{1,3}: \mathscr{N}(K)$ has four connected components

$$
\mathscr{N}(K)_{\epsilon \delta}:=\{t=\epsilon z, \delta t>0\}, \quad \epsilon, \delta \in\{ \pm 1\} .
$$

The closure $\overline{\mathscr{N}(K)}$ of $\mathscr{N}(K)$ is the set $\{|t|=|z|\}$, which is not a manifold, because of the crossing of the null hyperplanes $\{t= \pm z\}$ at $t=z=0$. Horizons of this type are referred to as bifurcate Killing horizons.

A very similar behavior is met in the extended Schwarzschild space-time: the set $\{r=2 m\}$ is a null hypersurface $\mathscr{E}$, the Schwarzschild event horizon. The stationary Killing vector $X=\partial_{t}$ extends to a Killing vector $\hat{X}$, which becomes tangent to and null on $\mathscr{E}$ in the extended space-time, except at the bifurcation sphere right in the middle of Figure 3.1, where $\hat{X}$ vanishes. 
A last noteworthy example in Minkowski space-time $\mathbb{R}^{1,3}$ is provided by the Killing vector

$$
X=y \partial_{t}+t \partial_{y}+x \partial_{y}-y \partial_{x}=y \partial_{t}+(t+x) \partial_{y}-y \partial_{x} .
$$

Thus, $X$ is the sum of a boost $y \partial_{t}+t \partial_{y}$ and a rotation $x \partial_{y}-y \partial_{x}$. Note that $X$ vanishes if and only if

$$
y=t+x=0,
$$

which is a two-dimensional null submanifold of $\mathbb{R}^{1,3}$. The vanishing set of the Lorentzian length of $X$,

$$
g(X, X)=(t+x)^{2}=0,
$$

is a null hyperplane in $\mathbb{R}^{1,3}$. It follows that, e.g., the set

$$
\{t+x=0, y>0, t>0\}
$$

is a Killing horizon with respect to two different Killing vectors, the boost Killing vector $x \partial_{t}+t \partial_{x}$, and the Killing vector (3.12).

3.3.1. Surface gravity. The surface gravity $\kappa$ of a Killing horizon is defined by the formula

$$
d(g(X, X))=-2 \kappa X^{b},
$$

where $X^{b}$ is the one-form metrically dual to $X$, i.e., $X^{b}=g_{\mu \nu} X^{\nu} d x^{\mu}$. Two comments are in order: First, since $g(X, X)=0$ on $\mathscr{N}(X)$, the differential of $g(X, X)$ annihilates $T \mathscr{N}(X)$. Now, simple algebra shows that a one-form annihilating a null hypersurface is proportional to $g(\ell, \cdot)$, where $\ell$ is any null vector tangent to $\mathscr{N}$ (those are defined uniquely up to a proportionality factor; see Section 7.1). We thus obtain that $d(g(X, X))$ is proportional to $X^{b}$, whence (3.13). Next, the name surface gravity stems from the following: using the Killing equations (3.10) and (3.13), one has

$$
X^{\mu} \nabla_{\mu} X^{\sigma}=-X^{\mu} \nabla^{\sigma} X_{\mu}=\kappa X^{\sigma} .
$$

Since the left-hand side of (3.14) is the acceleration of the integral curves of $X$, the equation shows that, in a certain sense, $\kappa$ measures the gravitational field at the horizon.

A key property is that the surface gravity $\kappa$ is constant on bifurcate [181, p. 59] Killing horizons. Furthermore, $\kappa$ [158, Theorem 7.1] is constant for all Killing horizons, whether bifurcate or not, in space-times satisfying the dominant energy condition: this means that

$$
T_{\mu \nu} X^{\mu} Y^{\nu} \geq 0 \text { for causal future-directed vector fields } X \text { and } Y \text {. }
$$

As an example, consider the Killing vector $K$ of (3.11). We have

$$
d(g(K, K))=d\left(-z^{2}+t^{2}\right)=2(-z d z+t d t),
$$

which equals twice $K^{\mathrm{b}}$ on $\mathscr{N}(K)_{\epsilon \delta}$. On the other hand, for the Killing vector $X$ of (3.12) one obtains

$$
d(g(X, X))=2(t+x)(d t+d x),
$$

which vanishes on each of the Killing horizons $\{t=-x, y \neq 0\}$. This shows that the same null surface can have zero or nonzero values of surface gravity, depending upon which Killing vector has been chosen to calculate $\kappa$.

The surface gravity of black holes plays an important role in black hole thermodynamics; see [59] and the references therein. 
A Killing horizon $\mathscr{N}_{0}(X)$ is said to be degenerate, or extreme, if $\kappa$ vanishes throughout $\mathscr{N}_{0}(X)$; it is called nondegenerate if $\kappa$ has no zeros on $\mathscr{N}_{0}(X)$. Thus, the Killing horizons $\mathscr{N}(K)_{\epsilon \delta}$ are nondegenerate, while both Killing horizons of $X$ given by (3.12) are degenerate. The Schwarzschild black holes have surface gravity

$$
\kappa_{m}=\frac{1}{2 m} \text {. }
$$

So there are no degenerate black holes within the Schwarzschild family. In fact [110, there are no regular, degenerate, static vacuum black holes at all.

In Kerr space-times we have $\kappa=0$ if and only if $m=|a|$.

3.3.2. Average surface gravity. Following 223, near a smooth null hypersurface one can introduce Gaussian null coordinates, in which the metric takes the form

$$
g=r \varphi d v^{2}+2 d v d r+2 r \chi_{a} d x^{a} d v+h_{a b} d x^{a} d x^{b} .
$$

The hypersurface is given by the equation $\{r=0\}$. Let $S$ be any smooth compact cross-section of the horizon. Then the average surface gravity $\langle\kappa\rangle_{S}$ is defined as

$$
\langle\kappa\rangle_{S}=-\frac{1}{|S|} \int_{S} \varphi d \mu_{h},
$$

where $d \mu_{h}$ is the measure induced by the metric $h$ on $S$, and $|S|$ is the volume of $S$. We emphasize that this is defined regardless of whether or not the stationary Killing vector is tangent to the null generators of the hypersurface; on the other hand, $\langle\kappa\rangle_{S}$ coincides with $\kappa$ when $\kappa$ is constant and the Killing vector equals $\partial_{v}$.

3.4. Asymptotically flat metrics. In relativity one often needs to consider initial data on noncompact manifolds, with natural restrictions on the asymptotic geometry. The most commonly studied such examples are asymptotically flat manifolds, which model isolated gravitational systems. Now, there exist several ways of defining asymptotic flatness, all of them roughly equivalent in vacuum. We will adapt a Cauchy data point of view, as it appears to be the least restrictive; the discussion here will also be relevant for Section 5 .

So, a space-time $(\mathscr{M}, g)$ will be said to possess an asymptotically flat end if $\mathscr{M}$ contains a spacelike hypersurface $M_{\text {ext }}$ diffeomorphic to $\mathbb{R}^{n} \backslash B(R)$, where $B(R)$ is a coordinate ball of radius $R$. An end comes thus equipped with a set of Euclidean coordinates $\left\{x^{i}, i=1, \ldots, n\right\}$, and one sets $r=|x|:=\left(\sum_{i=1}^{n}\left(x^{i}\right)^{2}\right)^{1 / 2}$. One then assumes that there exists a constant $\alpha>0$ such that, in local coordinates on $M_{\text {ext }}$ obtained from $\mathbb{R}^{n} \backslash B(R)$, the metric $h$ induced by $g$ on $M_{\text {ext }}$, and the second fundamental form $K$ of $M_{\text {ext }}$ (compare (4.15) below), satisfy the fall-off conditions, for some $k>1$,

$$
h_{i j}-\delta_{i j}=O_{k}\left(r^{-\alpha}\right), \quad K_{i j}=O_{k-1}\left(r^{-1-\alpha}\right),
$$

where we write $f=O_{k}\left(r^{\beta}\right)$ if $f$ satisfies

$$
\partial_{k_{1}} \cdots \partial_{k_{\ell}} f=O\left(r^{\beta-\ell}\right), \quad 0 \leq \ell \leq k .
$$

In applications one needs $(h, K)$ to lie in a certain weighted Hölder or Sobolev space defined on $M$, with the former better suited for the treatment of the evolution as discussed in Section 64

\footnotetext{
${ }^{4}$ The analysis of elliptic operators such as the Laplacian on weighted Sobolev spaces was initiated by Nirenberg and Walker [230, (see also [20, 72, 202, 203, 204, 205, 215, 216, 217] as well
} 
3.5. Asymptotically flat stationary metrics. For simplicity we assume that the space-time is vacuum, though similar results hold in general under appropriate conditions on matter fields; see [30, 108] and the references therein.

Along any spacelike hypersurface $M$, a Killing vector field $X$ of $(\mathscr{M}, g)$ can be decomposed as

$$
X=N n+Y,
$$

where $Y$ is tangent to $M$, and $n$ is the unit future-directed normal to $M$. The fields $N$ and $Y$ are called Killing initial data, or KID for short. The vacuum field equations, together with the Killing equations, imply the following set of equations on $M$ :

$$
\begin{gathered}
D_{i} Y_{j}+D_{j} Y_{i}=2 N K_{i j}, \\
R_{i j}(h)+K^{k}{ }_{k} K_{i j}-2 K_{i k} K^{k}{ }_{j}-N^{-1}\left(\mathscr{L}_{Y} K_{i j}+D_{i} D_{j} N\right)=0,
\end{gathered}
$$

where $R_{i j}(h)$ is the Ricci tensor of $h$. These equations play an important role in the gluing constructions described in Section 5.3 ,

Under the boundary conditions (3.18), an analysis of these equations provides detailed information about the asymptotic behavior of $(N, Y)$. In particular one can prove that if the asymptotic region $M_{\text {ext }}$ is contained in a hypersurface $M$ satisfying the requirements of the positive energy theorem (see Section 5.2.1), and if $X$ is timelike along $M_{\text {ext }}$, then $\left(N, Y^{i}\right) \rightarrow_{r \rightarrow \infty}\left(A^{0}, A^{i}\right)$, where the $A^{\mu}$ 's are constants satisfying $\left(A^{0}\right)^{2}>\sum_{i}\left(A^{i}\right)^{2}[31,108$. Further, in the coordinates of (3.18),

$$
Y^{i}-A^{i}=O_{k}\left(r^{-\alpha}\right), \quad N-A^{0}=O_{k}\left(r^{-\alpha}\right) .
$$

As discussed in more detail in [32, in $h$-harmonic coordinates, and in, e.g., a maximal (i.e., mean curvature zero) time-slicing, the vacuum equations for $g$ form a quasi-linear elliptic system with diagonal principal part, with principal symbol identical to that of the scalar Laplace operator. It can be shown that, in this "gauge", all metric functions have a full asymptotic expansion in terms of powers of $\ln r$ and inverse powers of $r$. In the new coordinates we can in fact take

$$
\alpha=n-2 \text {. }
$$

By inspection of the equations one can further infer that the leading-order corrections in the metric can be written in the Schwarzschild form (3.8).

3.6. Domains of outer communications, event horizons. A key notion in the theory of asymptotically flat black holes is that of the domain of outer communications, defined for stationary space-times as follows: For $t \in \mathbb{R}$, let $\phi_{t}[X]: \mathscr{M} \rightarrow \mathscr{M}$ denote the one-parameter group of diffeomorphisms generated by $X$; we will write $\phi_{t}$ for $\phi_{t}[X]$ whenever ambiguities are unlikely to occur. Let $M_{\text {ext }}$ be as in Section 3.4, and assume that $X$ is timelike along $M_{\text {ext }}$. The exterior region $\mathscr{M}_{\text {ext }}$ and the domain of outer communications $\left\langle\left\langle\mathscr{M}_{\text {ext }}\right\rangle\right\rangle$ are then defined a:5

$$
\mathscr{M}_{\text {ext }}:=\bigcup_{t} \phi_{t}\left(M_{\text {ext }}\right), \quad\left\langle\left\langle\mathscr{M}_{\text {ext }}\right\rangle\right\rangle=I^{+}\left(\mathscr{M}_{\text {ext }}\right) \cap I^{-}\left(\mathscr{M}_{\text {ext }}\right) .
$$

as [71). A readable treatment of analysis on weighted spaces (not focusing on relativity) can be found in 235.

${ }^{5}$ See Section 2.3 .1 for the definition of $I^{ \pm}(\Omega)$. 

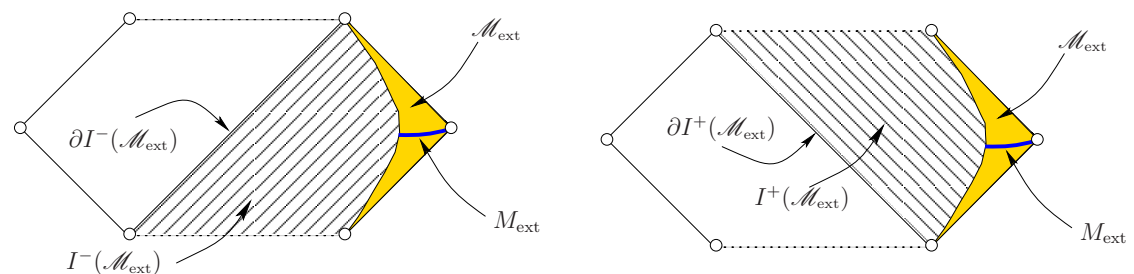

FiguRE 3.2. $M_{\text {ext }}, \mathscr{M}_{\text {ext }}$, together with the future and the past of $\mathscr{M}_{\text {ext }}$. One has $\mathscr{M}_{\text {ext }} \subset I^{ \pm}\left(\mathscr{M}_{\text {ext }}\right)$, even though this is not immediately apparent from the figure. The domain of outer communications is the intersection $I^{+}\left(\mathscr{M}_{\text {ext }}\right) \cap I^{-}\left(\mathscr{M}_{\text {ext }}\right)$; compare Figure 3.3 .

The black hole region $\mathscr{B}$ and the black hole event horizon $\mathscr{H}^{+}$are defined as (see Figures 3.2 and 3.3

$$
\mathscr{B}=\mathscr{M} \backslash I^{-}\left(\mathscr{M}_{\mathrm{ext}}\right), \quad \mathscr{H}^{+}=\partial \mathscr{B}
$$

The white hole region $\mathscr{W}$ and the white hole event horizon $\mathscr{H}^{-}$are defined as above after changing the time orientation:

$$
\mathscr{W}=\mathscr{M} \backslash I^{+}\left(\mathscr{M}_{\text {ext }}\right), \quad \mathscr{H}^{-}=\partial \mathscr{W} .
$$

It follows that the boundaries of $\left\langle\left\langle\mathscr{M}_{\text {ext }}\right\rangle\right\rangle$ are included in the event horizons. We set

$$
\mathscr{E}^{ \pm}=\partial\left\langle\left\langle\mathscr{M}_{\text {ext }}\right\rangle\right\rangle \cap I^{ \pm}\left(\mathscr{M}_{\text {ext }}\right), \quad \mathscr{E}=\mathscr{E}^{+} \cup \mathscr{E}^{-} .
$$

The sets $\mathscr{E}^{ \pm}$are achronal boundaries and so, as mentioned in Section 2, they are ruled by null geodesics, called generators.

In general, each asymptotically flat end of $\mathscr{M}$ determines a different domain of outer communications. Although there is considerable freedom in choosing the asymptotic region $M_{\text {ext }}$ giving rise to a particular end, it can be shown that $I^{ \pm}\left(\mathscr{M}_{\text {ext }}\right)$, and hence $\left\langle\left\langle\mathscr{M}_{\text {ext }}\right\rangle\right\rangle, \mathscr{H}^{ \pm}$and $\mathscr{E}^{ \pm}$, are independent of the choice of $M_{\text {ext }}$.

3.7. Uniqueness theorems. It is widely expected that the Kerr metrics provide the only stationary, regular, vacuum, four-dimensional black holes. In spite of many works on the subject (see [1, 66, 99, 226, 158, 168, 227, 252, 281 and the references therein), the question is far from being settled.

To describe the current state of affairs, some terminology is needed. A Killing vector $X$ is said to be complete if its orbits are complete; i.e., for every $p \in \mathscr{M}$, the orbit $\phi_{t}[X](p)$ of $X$ is defined for all $t \in \mathbb{R} . X$ is called stationary if it is timelike at large distances in the asymptotically flat region.

A key definition for the uniqueness theory is the following:

Definition 3.1. Let $(\mathscr{M}, g)$ be a space-time containing an asymptotically flat end $M_{\text {ext }}$, and let $X$ be a stationary Killing vector field on $\mathscr{M}$. We will say that $(\mathscr{M}, g, X)$ is $I^{+}$-regular if $X$ is complete, if the domain of outer communications $\left\langle\left\langle\mathscr{M}_{\text {ext }}\right\rangle\right\rangle$ is globally hyperbolic, and if $\left\langle\left\langle\mathscr{M}_{\text {ext }}\right\rangle\right\rangle$ contains a spacelike, connected, acausal hypersurface $M \supset M_{\text {ext }}$, the closure $\bar{M}$ of which is a topological manifold with boundary, consisting of the union of a compact set and of a finite number of 


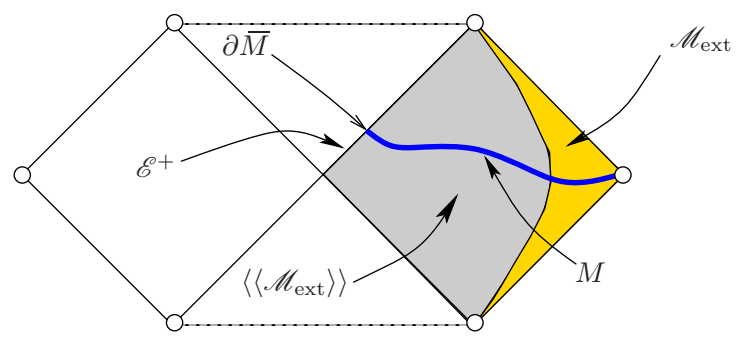

Figure 3.3. The hypersurface $M$ from the definition of $I^{+}$ regularity. To avoid ambiguities, we note that $\mathscr{M}_{\text {ext }}$ is a subset of $\left\langle\left\langle\mathscr{M}_{\text {ext }}\right\rangle\right\rangle$.

asymptotically flat ends, such that the boundary $\partial \bar{M}:=\bar{M} \backslash M$ satisfies

$$
\partial \bar{M} \subset \mathscr{E}^{+}
$$

(see (3.26) ) with $\partial \bar{M}$ meeting every generator of $\mathscr{E}^{+}$precisely once; see Figure 3.3 .

Some comments might be helpful. First, one requires completeness of the orbits of the stationary Killing vector because one needs an action of $\mathbb{R}$ on $\mathscr{M}$ by isometries. Next, one requires global hyperbolicity of the domain of outer communications to guarantee its simple connectedness and to avoid causality violations. Further, the existence of a well-behaved spacelike hypersurface gives reasonable control of the geometry of $\left\langle\left\langle\mathscr{M}_{\text {ext }}\right\rangle\right\rangle$ and is a prerequisite to any elliptic PDE analysis, as is extensively needed for the problem at hand. The existence of compact crosssections of the future event horizon $\mathscr{E}^{+}$prevents singularities on the future part of the boundary of the domain of outer communications and eventually guarantees the smoothness of that boundary.

The event horizon in a stationary space-time will be said to be rotating if the stationary Killing vector is not tangent to the generators of the horizon; it will be said to be mean nondegenerate if $\langle\kappa\rangle_{\partial \bar{M}} \neq 0$ (compare (3.17)). The proof of the following can be found in [99] in the mean nondegenerate case and in [109] in the degenerate rotating one:

Theorem 3.2. Let $(\mathscr{M}, g)$ be an $I^{+}$-regular, vacuum, analytic, asymptotically flat, four-dimensional stationary space-time. If $\mathscr{E}^{+}$is connected and either mean nondegenerate or rotating, then $\left\langle\left\langle\mathscr{M}_{\mathrm{ext}}\right\rangle\right\rangle$ is isometric to the domain of outer communications of a Kerr space-time.

Theorem 3.2 finds its roots in work by Carter [66] and Robinson [252], with further key steps due to Hawking [152] and Sudarsky and Wald 268. It should be emphasized that the hypotheses of connectedness, analyticity and nondegeneracy in the case of nonrotating configurations are highly unsatisfactory, and one believes that they are not needed for the conclusion. Recent progress on the connectedness question has been done by Neugebauer and Hennig [226], who excluded two-component configurations under a nondegeneracy condition whose meaning remains to be explored; see also [196, 282 for previous results.

The analyticity restriction has been removed by Alexakis, Ionescu and Klainerman in 1 for near-Kerrian configurations, but the general case remains open. 
Partial results concerning uniqueness of higher-dimensional black holes have been obtained by Hollands and Yazadjiev [163, 161, 162; compare [58, 149, 150, 224].

\section{The Cauchy problem}

The component version of the vacuum Einstein equations with cosmological constant $\Lambda$ (2.8) reads

$$
G_{\alpha \beta}+\Lambda g_{\alpha \beta}=0,
$$

where $G_{\alpha \beta}$ is the Einstein tensor defined as

$$
G_{\alpha \beta}:=R_{\alpha \beta}-\frac{1}{2} R g_{\alpha \beta},
$$

while $R_{\alpha \beta}$ is the Ricci tensor and $R$ the scalar curvature. We will refer to those equations as the vacuum Einstein equations, regardless of whether or not the cosmological constant vanishes, and in this work we will mostly assume $\Lambda=0$. Taking the trace of (4.1), one obtains

$$
R=\frac{2(n+1)}{n-1} \Lambda
$$

where, as elsewhere, $n+1$ is the dimension of space-time. This leads to the following equivalent version of (4.1):

$$
\text { Ric }=\frac{2 \Lambda}{n-1} g .
$$

Thus the Ricci tensor of the metric is proportional to the metric. Pseudo-Riemannian manifolds with metrics satisfying equation (4.4) are called Einstein manifolds in the mathematical literature; see, e.g., 43 .

Given a manifold $\mathscr{M}$, equation (4.1) or, equivalently, equation (4.4) forms a system of second-order partial differential equations for the metric, linear in the second derivatives of the metric, with coefficients which are rational functions of the $g_{\alpha \beta}$ 's, quadratic in the first derivatives of $g$, again with coefficients rational in $g$. Equations linear in the highest-order derivatives are called quasi-linear; hence the vacuum Einstein equations constitute a second-order system of quasi-linear partial differential equations for the metric $g$.

In the discussion above we assumed that the manifold $\mathscr{M}$ has been given. In the evolutionary point of view, which we adapt in most of this work, all space-times of main interest have topology $\mathbb{R} \times M$, where $M$ is an $n$-dimensional manifold carrying initial data. Thus, solutions of the Cauchy problem (as defined precisely by Theorem 6.1 below) have topology and differential structure which are determined by the initial data. As will be discussed in more detail in Section 6.1, the spacetimes obtained by evolution of the data are sometimes extendible; there is then a lot of freedom in the topology of the extended space-time, and we are not aware of conditions which would guarantee uniqueness of the extensions. So in the evolutionary approach the manifold is best thought of as being given a priori, namely $\mathscr{M}=\mathbb{R} \times M$, but it should be kept in mind that there is no a priori known natural time-coordinate which can be constructed by evolutionary methods, and which leads to the decomposition $\mathscr{M}=\mathbb{R} \times M$.

Now, there exist standard classes of partial differential equations which are known to have good properties. They are determined by looking at the algebraic 
properties of those terms in the equations which contain derivatives of highest order, in our case of order two. Inspection of (4.1) shows that this equation does not fall in any of the standard classes, such as hyperbolic, parabolic, or elliptic. In retrospect this is not surprising, because equations in those classes typically lead to unique solutions. On the other hand, given any solution $g$ of the Einstein equations (4.4) and any diffeomorphism $\Phi$, the pull-back metric $\Phi^{*} g$ is also a solution of (4.4), so whatever uniqueness there might be will hold only up to diffeomorphisms. An alternative way of describing this, often found in the physics literature, is the following: suppose that we have a matrix $g_{\mu \nu}(x)$ of functions satisfying (4.1) in some coordinate system $x^{\mu}$. If we perform a coordinate change $x^{\mu} \rightarrow y^{\alpha}\left(x^{\mu}\right)$, then the matrix of functions $\bar{g}_{\alpha \beta}(y)$ defined as

$$
g_{\mu \nu}(x) \rightarrow \bar{g}_{\alpha \beta}(y)=g_{\mu \nu}(x(y)) \frac{\partial x^{\mu}}{\partial y^{\alpha}} \frac{\partial x^{\nu}}{\partial y^{\beta}}
$$

will also solve (4.1) if the $x$-derivatives there are replaced by $y$-derivatives. This property is known under the name of diffeomorphism invariance, or coordinate invariance, of the Einstein equations. Physicists say that "the diffeomorphism group is the gauge group of Einstein's theory of gravitation."

Somewhat surprisingly, Choquet-Bruhat 140] proved in 1952 that there exists a set of hyperbolic equations underlying (4.2). This proceeds by the introduction of so-called harmonic coordinates, to which we turn our attention in the next section.

\subsection{The local evolution problem.}

4.1.1. Wave-coordinates. A set of coordinates $\left\{y^{\mu}\right\}$ is called harmonic if each of the functions $y^{\mu}$ satisfies

$$
\square_{g} y^{\mu}=0
$$

where $\square_{g}$ is the d'Alembertian associated with $g$ acting on scalars

$$
\square_{g} f:=\operatorname{tr}_{g} \operatorname{Hess} f=\frac{1}{\sqrt{|\operatorname{det} g|}} \partial_{\mu}\left(\sqrt{|\operatorname{det} g|} g^{\mu \nu} \partial_{\nu} f\right) \text {. }
$$

One also refers to these as wave-coordinates. Assuming that (4.6) holds, (4.4) can be written as

$$
\begin{aligned}
0=\hat{E}^{\alpha \beta}:= & \square_{g} g^{\alpha \beta}-g^{\epsilon \phi}\left(2 g^{\gamma \delta} \Gamma_{\gamma \epsilon}^{\alpha} \Gamma_{\delta \phi}^{\beta}+\left(g^{\alpha \gamma} \Gamma_{\gamma \delta}^{\beta}+g^{\beta \gamma} \Gamma_{\gamma \delta}^{\alpha}\right) \Gamma_{\epsilon \phi}^{\delta}\right) \\
& -\frac{4 \Lambda}{n-1} g^{\alpha \beta} .
\end{aligned}
$$

Here the $\Gamma_{\beta \gamma}^{\alpha}$ 's should be calculated in terms of the $g_{\alpha \beta}$ 's and their derivatives as in (2.3), and the wave operator $\square_{g}$ is as in (4.7). So, in wave-coordinates, the Einstein equation forms a second-order quasi-linear wave-type system of equations (4.8) for the metric functions $g^{\alpha \beta}$. (This can of course be rewritten as a set of quasi-linear equations for the $g_{\alpha \beta}$ 's by algebraic manipulations.)

The standard theory of hyperbolic PDEs 136 gives 6

\footnotetext{
${ }^{6}$ If $k$ is an integer, then the Sobolev spaces $H_{\mathrm{loc}}^{k}$ are defined as spaces of functions which are in $L^{2}(K)$ for any compact set $K$, with their distributional derivatives up to order $k$ also in $L^{2}(K)$. In the results presented here, one can actually allow noninteger $k$ 's; the spaces $H_{\text {loc }}^{k}$ are then defined rather similarly using the Fourier transformation.
} 
Theorem 4.1. For any initial data

$$
g^{\alpha \beta}\left(0, y^{i}\right) \in H_{l o c}^{k+1}, \quad \partial_{0} g^{\alpha \beta}\left(0, y^{i}\right) \in H_{l o c}^{k}, \quad k>n / 2,
$$

prescribed on an open subset $\mathscr{O} \subset\{0\} \times \mathbb{R}^{n} \subset \mathbb{R} \times \mathbb{R}^{n}$ there exists a unique solution $g^{\alpha \beta}$ of (4.8) defined on an open neighborhood $\mathscr{U} \subset \mathbb{R} \times \mathbb{R}^{n}$ of $\mathscr{O}$. The set $\mathscr{U}$ can be chosen so that $(\mathscr{U}, g)$ is globally hyperbolic with Cauchy surface $\mathscr{O}$.

Remark 4.2. The results in [188, 189, 190, 267] and the references therein allow one to reduce the differentiability threshold above.

Equation (4.8) would establish the hyperbolic, evolutionary character of the Einstein equations if not for the following problem: Given initial data for an equation as in (4.8) there exists a unique solution, at least for some short time. But there is a priori no reason to expect that the solution will satisfy (4.6); if it does not, then a solution of (4.8) will not solve the Einstein equation. In fact, if we set

$$
\lambda^{\mu}:=\square_{g} y^{\mu},
$$

then

$$
R^{\alpha \beta}=\frac{1}{2}\left(\hat{E}^{\alpha \beta}-\nabla^{\alpha} \lambda^{\beta}-\nabla^{\beta} \lambda^{\alpha}\right)+\frac{2 \Lambda}{n-1} g^{\alpha \beta},
$$

so that it is precisely the vanishing (or not) of $\lambda$ which decides whether or not a solution of (4.8) is a solution of the vacuum Einstein equations.

This problem has been solved by Choquet-Bruhat [140. The key observation is that (4.11) and the Bianchi identity imply a wave equation for the $\lambda^{\alpha}$ 's. In order to see that, recall the twice-contracted Bianchi identity (2.10):

$$
\nabla_{\alpha}\left(R^{\alpha \beta}-\frac{R}{2} g^{\alpha \beta}\right)=0 .
$$

Assuming that (4.8) holds, one finds

$$
\begin{aligned}
0 & =-\nabla_{\alpha}\left(\nabla^{\alpha} \lambda^{\beta}+\nabla^{\beta} \lambda^{\alpha}-\nabla_{\gamma} \lambda^{\gamma} g^{\alpha \beta}\right) \\
& =-\left(\square_{g} \lambda^{\beta}+R^{\beta}{ }_{\alpha} \lambda^{\alpha}\right) .
\end{aligned}
$$

This shows that $\lambda^{\alpha}$ necessarily satisfies the second-order hyperbolic system of equations

$$
\square_{g} \lambda^{\beta}+R_{\alpha}^{\beta} \lambda^{\alpha}=0 .
$$

Now, it is a standard fact in the theory of hyperbolic equations that we will have

$$
\lambda^{\alpha} \equiv 0
$$

on the domain of dependence $\mathscr{D}(\mathscr{O})$, provided that both $\lambda^{\alpha}$ and its derivatives vanish at $\mathscr{O}$. To see how these initial conditions on $\lambda^{\alpha}$ can be ensured, it is convenient to assume that $y^{0}$ is the coordinate along the $\mathbb{R}$ factor of $\mathbb{R} \times \mathbb{R}^{n}$, so that the initial data surface $\{0\} \times \mathscr{O}$ is given by the equation $y^{0}=0$. We have

$$
\begin{aligned}
\square_{g} y^{\alpha} & =\frac{1}{\sqrt{|\operatorname{det} g|}} \partial_{\beta}\left(\sqrt{|\operatorname{det} g|} g^{\beta \gamma} \partial_{\gamma} y^{\alpha}\right) \\
& =\frac{1}{\sqrt{|\operatorname{det} g|}} \partial_{\beta}\left(\sqrt{|\operatorname{det} g|} g^{\beta \alpha}\right) .
\end{aligned}
$$


Clearly, a necessary condition for the vanishing of $\square_{g} y^{\alpha}$ is that it vanishes at $y^{0}=0$, and this allows us to calculate some time derivatives of the metric in terms of space ones:

$$
\partial_{0}\left(\sqrt{|\operatorname{det} g|} g^{0 \alpha}\right)=-\partial_{i}\left(\sqrt{|\operatorname{det} g|} g^{i \alpha}\right) .
$$

This implies that the initial data (4.9) for the equation (4.8) cannot be chosen arbitrarily if we want both (4.8) and the Einstein equation to be simultaneously satisfied.

Now, there is still freedom left in choosing the wave-coordinates. Using this freedom, one can show that there is no loss of generality in assuming that on the initial hypersurface $\left\{y^{0}=0\right\}$ we have

$$
g^{00}=-1, \quad g^{0 i}=0,
$$

and this choice simplifies the algebra considerably. Equation (4.12) determines the time-derivatives $\left.\partial_{0} g^{0 \mu}\right|_{\left\{y^{0}=0\right\}}$ needed in Theorem 4.1 once $\left.g_{i j}\right|_{\left\{y^{0}=0\right\}}$ and $\left.\partial_{0} g_{i j}\right|_{\left\{y^{0}=0\right\}}$ are given. So, from this point of view, the essential initial data for the evolution problem are the space metric

$$
h:=g_{i j} d y^{i} d y^{j}
$$

together with its time-derivatives.

It turns out that further constraints arise from the requirement of the vanishing of the derivatives of $\lambda$. Supposing that (4.12) holds at $y^{0}=0$ (equivalently, supposing that $\lambda$ vanishes on $\left\{y^{0}=0\right\}$ ), we then have

$$
\partial_{i} \lambda^{\alpha}=0
$$

on $\left\{y^{0}=0\right\}$. To obtain the vanishing of all derivatives initially, it remains to ensure that some transverse derivative vanishes. A convenient transverse direction is provided by the field $n$ of unit timelike normals to $\left\{y^{0}=0\right\}$, and the vanishing of $\nabla_{n} \lambda^{\alpha}$ is guaranteed by requiring that

$$
\left(G_{\mu \nu}+\Lambda g_{\mu \nu}\right) n^{\mu}=0 .
$$

This follows by simple algebra from the equation $\hat{E}_{\alpha \beta}=0$ and (4.11),

$$
G_{\mu \nu}+\Lambda g_{\mu \nu}=-\left(\nabla_{\mu} \lambda_{\nu}+\nabla_{\nu} \lambda_{\mu}-\nabla^{\alpha} \lambda_{\alpha} g_{\mu \nu}\right),
$$

using that $\left.\lambda_{\mu}\right|_{y^{0}=0}=\left.\partial_{i} \lambda_{\mu}\right|_{y^{0}=0}=0$.

Equations (4.14) are called the Einstein constraint equations and will be discussed in detail in Section 5 .

Summarizing, we have proved:

Theorem 4.3. Under the hypotheses of Theorem 4.1, suppose that the initial data (4.9) satisfy (4.12), (4.13) as well as the constraint equations (4.14). Then the metric given by Theorem 4.1 satisfies the vacuum Einstein equations.

4.2. Cauchy data. In Theorem 4.1 we consider initial data given in a single coordinate patch $\mathscr{O} \subset \mathbb{R}^{n}$. This suffices for applications such as the Lindblad-Rodnianski stability theorem discussed in Section 6.4 below, where $\mathscr{O}=\mathbb{R}^{n}$. But a correct geometric picture is to start with an $n$-dimensional hypersurface $M$, and prescribe initial data there; the case where $M$ is $\mathscr{O}$ is thus a special case of this construction. At this stage there are two attitudes one may wish to adopt: the first is that $M$ is a 
subset of the space-time $\mathscr{M}$; this is essentially what we assumed in Section 4.1. The alternative is to consider $M$ as a manifold of its own, equipped with an embedding

$$
i: M \rightarrow \mathscr{M} \text {. }
$$

The most convenient approach is to go back and forth between those points of view, and this is the strategy that we will follow.

A vacuum initial data set $(M, h, K)$ is a triple, where $M$ is an $n$-dimensional manifold, $h$ is a Riemannian metric on $M$, and $K$ is a symmetric two-covariant tensor field on $M$. Further, $(h, K)$ are supposed to satisfy the vacuum constraint equations that result from (4.14) and which are written explicitly in terms of $K$ and $h$ in Section 5.1. Here the tensor field $K$ will eventually become the second fundamental form of $M$ in the resulting space-time $\mathscr{M}$, obtained by evolving the initial data. Recall that the second fundamental form of a spacelike hypersurface $M$ is defined as

$$
\forall X \in T M \quad K(X, Y)=g\left(\nabla_{X} n, Y\right)
$$

where $n$ is the future-pointing unit normal to $M$, and $K$ is often referred to as the extrinsic curvature tensor of $M$ in the relativity literature. Specifying $K$ is equivalent to prescribing the time-derivatives of the space-part $g_{i j}$ of the resulting space-time metric $g$; this can be seen as follows: Suppose, indeed, that a spacetime $(\mathscr{M}, g)$ has been constructed (not necessarily vacuum) such that $K$ is the extrinsic curvature tensor of $M$ in $(\mathscr{M}, g)$. Consider any domain of coordinates $\mathscr{O} \subset M$, and construct coordinates $y^{\mu}$ in a space-time neighborhood $\mathscr{U}$ such that $M \cap \mathscr{U}=\mathscr{O}$; those coordinates could be wave-coordinates obtained by solving the wave equations (4.6), but this is not necessary at this stage. Since $y^{0}$ is constant on $M$, the one-form $d y^{0}$ annihilates $T M \subset T \mathscr{M}$, as does the one-form $g(n, \cdot)$. Since $M$ has codimension one, it follows that $d y^{0}$ must be proportional to $g(n, \cdot)$ :

$$
n_{\alpha} d y^{\alpha}=n_{0} d y^{0}
$$

on $\mathscr{O}$. The normalization $-1=g(n, n)=g^{\mu \nu} n_{\mu} n_{\nu}=g^{00}\left(n_{0}\right)^{2}$ gives

$$
n_{\alpha} d y^{\alpha}=\frac{1}{\sqrt{\left|g^{00}\right|}} d y^{0}
$$

We then have, by 4.15),

$$
K_{i j}=-\frac{1}{2} g^{0 \sigma}\left(\partial_{j} g_{\sigma i}+\partial_{i} g_{\sigma j}-\partial_{\sigma} g_{i j}\right) n_{0}
$$

This shows that the knowledge of $g_{\mu \nu}$ and $\partial_{0} g_{i j}$ at $\left\{y^{0}=0\right\}$ allows one to calculate $K_{i j}$. Reciprocally, (4.16) can be rewritten as

$$
\partial_{0} g_{i j}=\frac{2}{g^{00} n_{0}} K_{i j}+\text { terms determined by the } g_{\mu \nu} \text { 's and their space-derivatives , }
$$

so that the knowledge of the $g_{\mu \nu}$ 's and of the $K_{i j}$ 's at $y^{0}=0$ allows one to calculate $\partial_{0} g_{i j}$. Thus, $K_{i j}$ is the geometric counterpart of the $\partial_{0} g_{i j}$ 's.

4.3. Solutions global in space. In order to globalize the existence Theorem 4.1 in space, the key point is to show that two solutions differing only by the values $\left.g_{0 \alpha}\right|_{\left\{y^{0}=0\right\}}$ are (locally) isometric. So suppose that $g$ and $\tilde{g}$ both solve the vacuum Einstein equations in a globally hyperbolic region $\mathscr{U}$, with the same Cauchy data 
$(h, K)$ on $\mathscr{O}:=\mathscr{U} \cap M$. One can then introduce wave coordinates in a globally hyperbolic neighborhood of $\mathscr{O}$ both for $g$ and $\tilde{g}$, satisfying (4.13), by solving

$$
\square_{g} y^{\mu}=0, \quad \square_{\tilde{g}} \tilde{y}^{\mu}=0,
$$

with the same initial data for $y^{\mu}$ and $\tilde{y}^{\mu}$. Transforming both metrics to their respective wave-coordinates, one obtains two solutions of the reduced equation (4.8) with the same initial data.

The question then arises whether the resulting metrics will be sufficiently differentiable to apply the uniqueness part of Theorem 4.1. Now, the metrics obtained so far are in a space $C^{1}\left([0, T], H^{s}\right)$, where the Sobolev space $H^{s}$ involves the spacederivatives of the metric. The initial data for the solutions $y^{\mu}$ or $\tilde{y}^{\mu}$ of (4.17) may be chosen to be in $H^{s+1} \times H^{s}$. However, a rough inspection of (4.17) shows that the resulting solutions will be only in $C^{1}\left([0, T], H^{s}\right)$, because of the low regularity of the metric. But then (4.5) implies that the transformed metrics will be in $C^{1}\left([0, T], H^{s-1}\right)$, and uniqueness can only be invoked provided that $s-1>n / 2+1$, which is one degree of differentiability more than what was required for existence. This was the state of affairs for some fifty-five years until the following simple argument of Planchon and Rodnianski [240]: To make it clear that the functions $y^{\mu}$ are considered to be scalars in (4.17), we shall write $y$ for $y^{\mu}$. Commuting derivatives with $\square_{g}$ one finds, for metrics satisfying the vacuum Einstein equations,

$$
\square_{g} \nabla_{\alpha} y=\nabla_{\mu} \nabla^{\mu} \nabla_{\alpha} y=\left[\nabla_{\mu} \nabla^{\mu}, \nabla_{\alpha}\right] y=\underbrace{R_{\alpha \mu}^{\sigma \mu}}_{=R_{\alpha}^{\sigma}=0} \nabla_{\sigma} y=0 .
$$

Commuting once more, one obtains an evolution equation for the field $\psi_{\alpha \beta}:=$ $\nabla_{\alpha} \nabla_{\beta} y$ :

$$
\square_{g} \psi_{\alpha \beta}+\underbrace{\nabla_{\sigma} R_{\beta}{ }^{\lambda}{ }_{\alpha}{ }^{\sigma}}_{=0} \nabla_{\lambda} y+2 R_{\beta}{ }^{\lambda}{ }_{\alpha}{ }^{\sigma} \psi_{\sigma \lambda}=0,
$$

where the underbraced term vanishes, for vacuum metrics, by a contracted Bianchi identity. So the most offending term in this equation for $\psi_{\alpha \beta}$, involving three derivatives of the metric, disappears when the metric is vacuum. Standard theory of hyperbolic PDEs shows now that the functions $\nabla_{\alpha} \nabla_{\beta} y$ are in $C^{1}\left([0, T], H^{s-1}\right)$, hence $y \in C^{1}\left([0, T], H^{s+1}\right)$, and the transformed metrics are regular enough to invoke uniqueness without having to increase $s$.

Suppose, now, that an initial data set $(M, h, K)$ as in Theorem 4.1 is given. Covering $M$ by coordinate neighborhoods $\mathscr{O}_{p}, p \in M$, one can use Theorem 4.1 to construct globally hyperbolic developments $\left(\mathscr{U}_{p}, g_{p}\right)$ of $\left(\mathscr{U}_{p}, h, K\right)$. By the argument just given, the metrics so obtained will coincide, after performing a suitable coordinate transformation, wherever simultaneously defined. This allows one to patch the $\left(\mathscr{U}_{p}, g_{p}\right)$ 's together to a globally hyperbolic Lorentzian manifold, with Cauchy surface $M$. Thus:

Theorem 4.4. Any vacuum initial data set $(M, h, K)$ of differentiability class $H^{s+1} \times H^{s}, s>n / 2$, admits a globally hyperbolic development.

The solutions are locally unique, in a sense made clear by the proof. The important question of uniqueness in the large will be addressed in Section 6.1. 


\section{INITIAL DATA SETS}

We now turn our attention to an analysis of the constraint equations, returning to the evolution problem in Section 6 .

An essential part of the mathematical analysis of the Einstein field equations of general relativity is the rigourous formulation of the Cauchy problem, which is a means to describe solutions of a dynamical theory via the specification of initial data and the evolution of that data. In this section we will be concerned mainly with the initial data sets for the Cauchy problem, which have to satisfy the relativistic constraint equations (4.14). This leads to the following questions: What are the sets of allowable initial data? Is it possible to parameterize them in a useful way? What global properties of the space-time can be seen in the initial data sets? How does one engineer initial data so that the associated space-time has some specific properties?

5.1. The constraint equations. As explained in Section 4.2 an initial data set for a vacuum space-time consists of an $n$-dimensional manifold $M$ together with a Riemannian metric $h$ and a symmetric tensor $K$. In the nonvacuum case we also have a collection of nongravitational fields which we collectively label $\mathcal{F}$ (usually these are sections of a bundle over $M$ ). We have already seen the relativistic vacuum constraint equations expressed as the vanishing of the normal components of the Einstein equations (4.14). Now, if $h$ is the metric induced on a spacelike hypersurface in a Lorentzian manifold, it has its own curvature tensor $R_{j k \ell}^{i}$. If we denote by $K_{i j}$ the second fundamental form of $M$ in $\mathscr{M}$, and by $\mathcal{R}^{i}{ }_{j k \ell}$ the space-time curvature tensor, the Gauss-Codazzi equations provide the following relationships:

$$
\begin{gathered}
R_{j k \ell}^{i}=\mathcal{R}^{i}{ }_{j k \ell}+K^{i}{ }_{\ell} K_{j k}-K^{i}{ }_{k} K_{j \ell}, \\
D_{i} K_{j k}-D_{j} K_{i k}=\mathcal{R}_{i j k \mu} n^{\mu} .
\end{gathered}
$$

Here $n$ is the timelike normal to the hypersurface, and we are using a coordinate system in which the $\partial_{i}$ 's are tangent to the hypersurface $M$.

Contractions of (5.1) - (5.2) and simple algebra allow one to reexpress (4.14) in the following form, where we have now allowed for the additional presence of nongravitational fields:

$$
\begin{aligned}
\operatorname{div} K-d(\operatorname{tr} K) & =8 \pi J, \\
R(h)-2 \Lambda-|K|_{h}^{2}+(\operatorname{tr} K)^{2} & =16 \pi \rho, \\
\mathcal{C}(\mathcal{F}, h) & =0,
\end{aligned}
$$

where $R(h)$ is the scalar curvature of the metric $h, J$ is the momentum density of the nongravitational fields, $\rho$ is the energy density 7 and $\mathcal{C}(\mathcal{F}, h)$ denotes the set of additional constraints that might come from the nongravitational part of the theory. The first of these equations is known as the momentum constraint and is a vector field equation on $M$. The second, a scalar equation, is referred to as the scalar, or Hamiltonian, constraint, while the last are collectively labeled the nongravitational constraints. These are what we shall henceforth call the Einstein

\footnotetext{
${ }^{7}$ If $\mathcal{T}$ is the stress-energy tensor of the nongravitational fields and $n$ denotes the unit timelike normal to a hypersurface $M$ embedded in a space-time, with induced data $(M, h, K, \mathcal{F})$, then $J=-\mathcal{T}(n, \cdot)$ and $\rho=\mathcal{T}(n, n)$. However, in terms of the initial data set itself we shall regard (5.3) -5.4) as the definitions of the quantities $J$ and $\rho$.
} 
constraint equations, or simply the constraint equations if ambiguities are unlikely to occur.

As an example, for the Einstein-Maxwell theory in $(3+1)$-dimensions, the nongravitational fields consist of the electric and magnetic vector fields $E$ and $B$. In this case we have $\rho=\frac{1}{2}\left(|E|_{h}^{2}+|B|_{h}^{2}\right), J=(E \times B)_{h}$, and we have the extra (nongravitational) constraints $\operatorname{div}_{h} E=0$ and $\operatorname{div}_{h} B=0$.

Equations (5.3) - (5.5) form an underdetermined system of partial differential equations. In the classical vacuum setting of $n=3$ dimensions, these are locally four equations for the twelve unknowns given by the components of the symmetric tensors $h$ and $K$. This section will focus primarily on the vacuum case with a zero cosmological constant.

The most successful approach so far for studying the existence and uniqueness of solutions to (5.3) - (5.5) is through the conformal method of Lichnerowicz [197, Choquet-Bruhat and York [81]. The idea is to introduce a set of unconstrained conformal data, which are freely chosen, and find $(h, K)$ by solving a system of determined partial differential equations. In the vacuum case with vanishing cosmological constant [81, the free conformal data consist of a manifold $M$, a Riemannian metric $\tilde{h}$ on $M$, a trace-free symmetric tensor $\tilde{\sigma}$, and the mean curvature function $\tau$. The initial data $(h, K)$ defined as

$$
\begin{aligned}
h & =\phi^{q} \tilde{h}, \quad q=\frac{4}{n-2}, \\
K & =\phi^{-2}(\tilde{\sigma}+\widetilde{\mathcal{D}} W)+\frac{\tau}{n} \phi^{q} \tilde{h},
\end{aligned}
$$

where $\phi$ is positive, will then solve (5.3) -(5.4) if and only if the function $\phi$ and the vector field $W$ solve the equations

$$
\begin{gathered}
\operatorname{div}_{\tilde{h}}(\widetilde{\mathcal{D}} W+\tilde{\sigma})=\frac{n-1}{n} \phi^{q+2} \tilde{D} \tau, \\
\Delta_{\tilde{h}} \phi-\frac{1}{q(n-1)} R(\tilde{h}) \phi+\frac{1}{q(n-1)}|\tilde{\sigma}+\tilde{\mathcal{D}} W|_{\tilde{h}}^{2} \phi^{-q-3}-\frac{1}{q n} \tau^{2} \phi^{q+1}=0 .
\end{gathered}
$$

We use the symbol $\tilde{D}$ to denote the covariant derivative of $\tilde{h} ; \widetilde{\mathcal{D}}$ is the conformal Killing operator:

$$
\widetilde{\mathcal{D}} W_{a b}=\tilde{D}_{a} W_{b}+\tilde{D}_{b} W_{a}-\frac{2}{n} \tilde{h}_{a b} \tilde{D}_{c} W^{c}
$$

Vector fields $W$ annihilated by $\mathcal{D}$ are called conformal Killing vector fields, and are characterized by the fact that they generate (perhaps local) conformal diffeomorphisms of $(M, h)$. The semilinear scalar equation (5.9) is often referred to as the Lichnerowicz equation.

Equations (5.8) - (5.9) form a determined system of equations for the $n+1$ functions $(\phi, W)$. The operator $\operatorname{div}_{\tilde{h}}(\widetilde{\mathcal{D}} \cdot)$ is a linear, formally selfadjoint, elliptic operator on vector fields. What makes the study of the system (5.8)-(5.9) difficult in general is the nonlinear coupling between the two equations.

The explicit choice of (5.6)-(5.7) is motivated by the two identities (for $\tilde{h}=\phi^{q} h$ )

$$
R(\tilde{h})=-\phi^{-q-1}\left(q(n-1) \Delta_{h} \phi-R(h) \phi\right),
$$


where $q=\frac{4}{n-2}$, which is the unique exponent that does not lead to supplementary $|D \phi|^{2}$ terms in (5.11), and

$$
D_{\tilde{h}}^{a}\left(\phi^{-2} B_{a b}\right)=\phi^{-q-2} D_{h}^{a} B_{a b},
$$

which holds for any trace-free tensor $B$. Equation (5.11) is the well-known identity relating the scalar curvatures of two conformally related metrics.

In the space-time evolution $(\mathscr{M}, g)$ of the initial data set $(M, h, K)$, the function $\tau=\operatorname{tr}_{h} K$ is the mean curvature of the hypersurface $M \subset \mathscr{M}$. The assumption that the mean curvature function $\tau$ is constant on $M$ significantly simplifies the analysis of the vacuum constraint equations because it decouples equations (5.8) and (5.9). One can then attempt to solve (5.8) for $W$, and then solve the Lichnerowicz equation (5.9).

The existence and uniqueness of solutions of this problem for constant mean curvature (CMC) data has been studied extensively. For compact manifolds this was exhaustively analysed by Isenberg [169, building upon a large amount of previous work 181, 197, 231, 287; the proof was simplified by Maxwell in 213. If we let $\mathcal{Y}([h])$ denote the Yamabe invariant of the conformal class $[h]$ of metrics determined by $h$ (see [193]), the result reads as follows:

Theorem 5.1 ([169]). Consider a smooth conformal initial data set $(\tilde{h}, \tilde{\sigma}, \tau)$ on a compact manifold $M$, with constant $\tau$. Then there always exists a solution $W$ of (5.8). Setting $\sigma=\widetilde{\mathcal{D}} W+\tilde{\sigma}$, the existence, or not, of a positive solution $\phi$ of the Lichnerowicz equation is shown in Table 5.1 .

TABLE 5.1. Existence of solutions in the conformal method for CMC data on compact manifolds.

\begin{tabular}{|l||c|c|c|c|}
\hline & $\sigma \equiv 0, \tau=0$ & $\sigma \equiv 0, \tau \neq 0$ & $\sigma \not \equiv 0, \tau=0$ & $\sigma \not \equiv 0, \tau \neq 0$ \\
\hline \hline $\mathcal{Y}([h])<0$ & No & Yes & No & Yes \\
\hline $\mathcal{Y}([h])=0$ & Yes & No & No & Yes \\
\hline $\mathcal{Y}([h])>0$ & No & No & Yes & Yes \\
\hline
\end{tabular}

More recently, work has been done on analyzing these equations for metrics of low differentiability 69, 213; this was motivated in part by recent work on the evolution problem for rough initial data [188, 189, 190, 267. Exterior boundary value problems for the constraint equations, with nonlinear boundary conditions motivated by black holes, were considered in 122,214 .

The conformal method easily extends to CMC constraint equations for some nonvacuum initial data, e.g., the Einstein-Maxwell system [169 where one obtains results very similar to those of Theorem [5.1. However, other important examples, such as the Einstein-scalar field system [77, 78, 79, 155, require more effort and are not as fully understood.

Conformal data close to being CMC (e.g., via a smallness assumption on $|\nabla \tau|$ ) are usually referred to as near-CMC. Classes of near-CMC conformal data solutions have been constructed [2, 76, 174, 175, and there is at least one example of a nonexistence theorem [178] for a class of near-CMC conformal data. However, due to the nonlinear coupling in the system (5.8)-(5.9), the question of existence 
for unrestricted choices of the mean curvature $\tau$ appears to be significantly more difficult, and until recently all results assumed strong restrictions on the gradient of $\tau$. The first general result in this context is due to Holst, Nagy, and Tsogtgerel [164, 165, who construct solutions with freely specified mean curvature in the presence of matter. In 212, Maxwell provides a sufficient condition, with no restrictions on the mean curvature, for the conformal method to generate solutions to the vacuum constraint equations on compact manifolds. As an application, Maxwell demonstrates the existence of a large class of solutions to the vacuum constraint equations with freely specified mean curvature. These results together represent a significant advance in our understanding of how the conformal method may be used to generate solutions of the vacuum constraint equations. However, the existence question for generic classes of large conformal data remains wide open; compare 211.

The analysis of the conformal constraint equations (5.8)-(5.9) discussed above proceeds either via the method of sub- and super-solutions (which is a barrier argument exploiting the maximum principle) or a perturbation or fixed point method. In [155, Hebey, Pacard and Pollack used the mountain pass lemma to analyse Lichnerowicz-type equations arising in certain cases of the Einstein-scalar field system. Such arguments may conceivably prove useful in studying (5.8)-(5.9) for general $\tau$ 's.

A natural question is whether the set of solutions to the constraint equations forms a manifold. This was first considered by Fischer and Marsden 139, who provided a Fréchet manifold structure; Banach manifold structures have been obtained in 102, and a Hilbert manifold structure (for asymptotically flat initial data sets) in 24 .

In [26, the reader will find a presentation of alternative approaches to constructing solutions of the constraints, covering work done up to 2003.

5.2. Mass inequalities. Among the deepest results in mathematical general relativity are the global mass inequalities for asymptotically flat manifolds. Those have been discussed extensively in the existing literature [50, 51, 566, 193, 253, 255, and therefore will only be given the minimum amount of attention, as needed for the remaining purposes of this work.

5.2.1. The Positive Mass Theorem. Using the coordinate system of (3.18), one defines the Arnowitt-Deser-Misner [15] mass of $(M, h)$ of an asymptotically flat end as

$$
m=\frac{1}{16 \pi} \lim _{r \rightarrow \infty} \int_{S_{r}} \sum_{i, j}\left(\frac{\partial h_{i j}}{\partial x^{i}}-\frac{\partial h_{i i}}{\partial x^{j}}\right) d S_{i} .
$$

Here $S_{r}$ is the coordinate sphere at radius $r$ and $\left.d S_{i}=\partial_{i}\right\rfloor d \mu$, and $d \mu$ is the Riemannian volume form of $h$. The factor $16 \pi$ is a matter of convention and is natural in space-dimension three. The integral converges to a finite, coordinateindependent limit if, for some $\alpha>\frac{n-2}{2}$,

$$
\left|h_{i j}-\delta_{i j}\right| \leq c r^{-\alpha}, \quad|\partial h| \leq c r^{-\alpha-1} \quad \text { and } \quad R(h) \in L^{1}\left(M_{\text {ext }}\right),
$$

with those conditions being essentially optimal [20, 92 .

For time-symmetric initial data the vacuum constraint equations (5.3)-(5.4) reduce to the condition that the metric $h$ be scalar-flat, i.e. $R(h)=0$. On the other hand, if one considers time-symmetric data for a nonvacuum space-time, then from 
(5.4) we see that the scalar curvature is twice the energy density of the matter fields. The nonnegativity of $R$, assuming a vanishing cosmological constant $\Lambda$, is then a consequence of the dominant energy condition for initial data (which follows from (3.15),

$$
\rho \geq|J|_{h},
$$

where $\rho$ and $J$ are defined in (5.3)-(5.4); see also Section 7.5. One checks that the dominant energy condition (3.15) holds on $\mathscr{M}$ if and only if (5.15) holds relative to each spacelike hypersurface in $\mathscr{M}$.

Now, the ADM mass is thought to represent the total mass of the system as viewed on $M$, which contains contributions from the matter fields, the gravitational field, as well as their binding energy. The long-standing question of its positivity was resolved by Schoen and Yau 257] in dimension three and is now known as the Positive Mass Theorem:

Theorem 5.2. Let $(M, h)$ be an asymptotically flat Riemannian manifold with nonnegative scalar curvature. Suppose that either $M$ is spin, or the dimension $n \leq 7$, or that $h$ is conformally flat. Then the total ADM mass $m$ satisfies $m \geq 0$, with equality if and only if $(M, h)$ is isometric to Euclidean space $\left(\mathbb{R}^{n}, \delta\right)$.

As remarked in the Introduction, this theorem, and its generalizations, stands as one of the cornerstones of mathematical relativity. Accessible introductions to the Positive Mass Theorem may be found in [51, 96, 193, 253. The restriction on the dimension arises from the use of area-minimizing hypersurfaces [253], which are known to sometimes possess singularities in higher dimensions, and it is expected that positivity is true in all dimensions. The Positive Mass Theorem was proven in all dimensions for conformally flat manifolds by Schoen and Yau [262] by a different argument, and in all dimensions for spin manifolds by Witten [285] (see also [20, 68, 236]). The result was generalized in [259, 260] (compare [255]) to asymptotically flat initial data sets $(M, h, K, \mathcal{F})$ satisfying the dominant energy condition (5.15).

5.2.2. Riemannian Penrose Inequality. An important generalization of the Positive Mass Theorem is given by the Riemannian Penrose Inequality.

Theorem 5.3. Let $(M, h)$ be a complete, smooth, asymptotically flat three-manifold with nonnegative scalar curvature with total mass $m$ and which has an outermost minimal surface $\Sigma_{0}$ of area $A_{0}$. Then

$$
m \geq \sqrt{\frac{A_{0}}{16 \pi}},
$$

with equality if and only if $(M, h)$ is isometric to the Schwarzschild metric $\left(\mathbb{R}^{3} \backslash\{0\},\left(1+\frac{m}{2|x|}\right)^{4} \delta\right)$ outside their respective outermost minimal surfaces.

Theorem 5.3 was first proved by Huisken and Ilmanen [167] under the restriction that $A_{0}$ is connected, or assuming instead that $A_{0}$ is the area of the largest connected component of $\Sigma_{0}$. The version above, with a proof that uses completely different methods, is due to Bray [48. The proofs are beautiful applications of 
geometric flows to a fundamental problem in relativity. A number of accessible reviews has been written on these important results, to which we refer the interested reader [50, 49, 51, 56, 208. A generalization of Theorem 5.3 to dimensions $n \leq 7$ has been established in [54.

One expects that some form of (5.16) holds for general relativistic initial data sets $(h, K)$ satisfying the dominant energy condition. A suggestion how one could prove this has been put forward by Bray and Khuri in [52, 53]; compare [57, 64, 141, 209. An inequality in the spirit of (5.16) but involving some further geometric constants, has been proved by Herzlich [157] in the Riemannian case.

5.2.3. Quasi-local mass. In the context of asymptotically flat space-times, there are well-defined global notions of mass and energy, and these are central to the celebrated Positive Mass Theorem discussed in Section 5.2.1 One would, however, like to have a well-defined useful local notion of mass or energy, with natural properties, e.g., monotonicity, that one has in other physical theories. Such a definition has been elusive despite a great deal of effort by many people, and this remains an important open problem. We refer the reader to the Living Reviews article by Szabados [269] for a survey and note that there have been interesting recent mathematical developments in the area [55, 218, 266, 278, 279, 280, not described in the currently available version of [269].

5.3. Applications of gluing techniques. Over the past 25 years, gluing techniques have become a standard tool in geometric analysis. Since the construction by Taubes of self-dual Yang-Mills connections on four-manifolds 274, which played a crucial role in Donaldson's construction of exotic smooth structures in four dimensions [127, gluing has been applied in important ways across a very wide range of areas. What gluing typically refers to is a construction in which solutions of a nonlinear partial differential equation or system, which correspond to some geometric quantity of interest, e.g., self-dual connections, are fused together to create new solutions. This is done by a mix of geometry and analysis in which one ultimately studies the linearization of the relevant PDEs, and in most cases one has to overcome the analytic degeneracy introduced in the gluing procedure. Thus, from an analytic point of view, gluing should be regarded as a singular perturbation method. Part of the usefulness of the technique lies in the fact that, away from the small set about which one fuses the two solutions, the new solution is very close to the original ones. The fact that the original solutions are usually not exactly preserved is a reflection of the fact that the relevant equations satisfy a unique continuation property: any two solutions which agree on an open set must agree everywhere. This is a well-known property for, say, a scalar semi-linear elliptic equation. The constraint equations, however, are underdetermined. As explained below, Corvino and Schoen have introduced gluing techniques which exploit this and show the failure (to a high degree) of unique continuation. This leads to localized gluings of initial data sets, which has proven very valuable.

5.3.1. The linearized constraint equations and KIDs. The starting point of gluing constructions for the constraint equations is the linearization of these equations about a given solution $(M, h, K)$. We let $\mathcal{P}_{(h, K)}^{*}$ denote the $L^{2}$ adjoint of the linearization of the constraint equations at this solution. Viewed as an operator acting 
on a scalar function $N$ and a vector field $Y, \mathcal{P}_{(h, K)}^{*}$ takes the explicit form [101]

$$
\mathcal{P}_{(h, K)}^{*}(N, Y)=\left(\begin{array}{l}
2\left(\nabla_{(i} Y_{j)}-\nabla^{l} Y_{l} g_{i j}-K_{i j} N+\operatorname{tr} K N g_{i j}\right) \\
\nabla^{l} Y_{l} K_{i j}-2 K^{l}{ }_{i} \nabla_{j)} Y_{l}+K^{q} \nabla_{q} \nabla^{l} g_{i j} \\
-\Delta N g_{i j}+\nabla_{i} \nabla_{j} N+\left(\nabla^{p} K_{l p} g_{i j}-\nabla_{l} K_{i j}\right) Y^{l} \\
-N \operatorname{Ric}(g)_{i j}+2 N K_{i}^{l} K_{j l}-2 N(\operatorname{tr} K) K_{i j}
\end{array}\right) .
$$

Now this operator does not, on first inspection, appear to be very "user friendly". However, our immediate concern is solely with its kernel, and the pairs $(N, Y)$ which lie in its kernel have a very straightforward geometric and physical characterization. In particular, let $\Omega$ be an open subset of $M$. By definition, the set of KIDs on $\Omega$, denoted $\mathscr{K}(\Omega)$, is the set of all solutions of the equation

$$
\mathcal{P}_{\left.(\gamma, K)\right|_{\Omega}}^{*}(N, Y)=0 .
$$

Such a solution $(N, Y)$, if nontrivial, generates a space-time Killing vector field in the domain of dependence of $\left(\Omega,\left.h\right|_{\Omega},\left.K\right|_{\Omega}\right)$ [219]; compare Section 3.4.

From a geometric point of view one expects that solutions with symmetries should be rare. This was made rigorous in [33, where it is shown that the generic behaviour among solutions of the constraint equations is the absence of KIDs on any open set. On the other hand, one should note that essentially every explicit solution has symmetries. In particular, both the flat initial data for Minkowski space and the initial data representing the constant time slices of Schwarzschild have KIDs.

5.3.2. Corvino's result. As we have already pointed out, the Einstein constraint equations form an underdetermined system of equations, and as such, it is unreasonable to expect that they (or their linearizations) should satisfy the unique continuation property. In 2000, Corvino established a gluing result for asymptotically flat metrics with zero scalar curvature which dramatically illustrated this point 113 . In the special case when one considers initial data with vanishing second fundamental form $K \equiv 0$, the momentum constraint equation (5.3) becomes trivial and the Hamiltonian constraint equation (5.3) reduces to simply $R(h)=0$, i.e., a scalar flat metric. Such initial data sets are referred to as time-symmetric because the space-time obtained by evolving them possesses a time-reversing isometry which leaves the initial data surface fixed. Beyond Euclidean space itself, the constant time slices of the Schwarzschild space-time form the most basic examples of asymptotically flat, scalar flat manifolds. One long-standing open problem 23, 263] in the field had been whether there exist scalar flat metrics on $\mathbb{R}^{n}$ which are not globally spherically symmetric but which are spherically symmetric in a neighborhood of infinity and hence, by Birkhoff's theorem, Schwarzschild there.

Corvino resolved this by showing that he could deform any asymptotically flat, scalar flat metric to one which is exactly Schwarzschild outside of a compact set.

Theorem 5.4 (113]). Let $(M, h)$ be a smooth Riemannian manifold with zero scalar curvature containing an asymptotically flat end $M_{\mathrm{ext}}=\{|x|>r>0\}$. Then there is an $R>r$ and a smooth metric $\bar{h}$ on $M$ with zero scalar curvature such that $\bar{h}$ is equal to $h$ in $M \backslash M_{\text {ext }}$ and $\bar{h}$ coincides on $\{|x|>R\}$ with the metric induced on a standard time-symmetric slice in the Schwarzschild solution. Moreover, the mass of $\bar{h}$ can be made arbitrarily close to that of $h$ by choosing $R$ sufficiently large. 
Underlying this result is a gluing construction where the deformation has compact support. The ability to do this is a reflection of the underdetermined nature of the constraint equations. In this setting, since $K \equiv 0$, the operator takes a much simpler form, as a two-covariant tensor-valued operator acting on a scalar function $u$ by

$$
\mathcal{P}^{*} u=-\left(\Delta_{h} u\right) h+\operatorname{Hess}_{h} u-u \operatorname{Ric}(h) .
$$

An elementary illustration of how an underdetermined system can lead to compactly supported solutions is given by the construction of compactly supported transversetraceless tensors on $\mathbb{R}^{3}$ in Appendix B of [114] (see also [29, 123]).

An additional challenge in proving Theorem 5.4 is the presence of KIDs on the standard slice of the Schwarzschild solution. If the original metric had ADM mass $m(h)$, a naive guess could be that the best fitting Schwarzschild solution would be the one with precisely the same mass. However the mass, and the coordinates of the center of mass, are in one-to-one correspondence with obstructions arising from KIDs. To compensate for this cokernel in the linearized problem, Corvino uses these $(n+1$ in dimension $n)$ degrees of freedom as effective parameters in the geometric construction. The final solution can be chosen to have its ADM mass arbitrarily close to the initial one.

The method uncovered in Corvino's thesis has been applied and extended in a number of important ways. The asymptotic simplicity model for isolated gravitational systems proposed by Penrose 237] has been very influential. This model assumes existence of smooth conformal completions to study global properties of asymptotically flat space-times. The question of existence of such vacuum spacetimes was open until Chruściel and Delay 100, and subsequently Corvino [114, used this type of gluing construction to demonstrate the existence of infinitedimensional families of vacuum initial data sets which evolve to asymptotically simple space-times. The extension of the gluing method to non-time-symmetric data was done in [101, 115. This allowed for the construction of space-times which are exactly Kerr outside of a compact set, as well as showed that one can specify other types of useful asymptotic behavior.

5.3.3. Conformal gluing. In [171, Isenberg, Mazzeo and Pollack developed a gluing construction for initial data sets satisfying certain natural nondegeneracy assumptions. The perspective taken there was to work within the conformal method, and thereby establish a gluing theorem for solutions of the determined system of PDEs given by (5.8) and (5.9). This was initially done only within the setting of constant mean curvature initial data sets and in dimension $n=3$ (the method was extended to all higher dimensions in 170]). The construction of [171] allowed one to combine initial data sets by taking a connected sum of their underlying manifolds, to add wormholes (by performing codimension-three surgery on the underlying, connected, three-manifold) to a given initial data set, and to replace arbitrary small neighborhoods of points in an initial data set with asymptotically hyperbolic ends.

In [172, this gluing construction was extended to only require that the mean curvature be constant in a small neighborhood of the point about which one wanted to perform a connected sum. This extension enabled the authors to show that one can replace an arbitrary small neighborhood of a generic point in any initial data set with an asymptotically flat end. Since it is easy to see that CMC solutions of the vacuum constraint equations exist on any compact manifold 284, this leads 
to the following result which asserts that there are no topological obstructions to asymptotically flat solutions of the constraint equations.

Theorem 5.5 ([172]). Let $M$ be any closed $n$-dimensional manifold, and let $p \in M$. Then $M \backslash\{p\}$ admits an asymptotically flat initial data set satisfying the vacuum constraint equations.

5.3.4. Initial data engineering. The gluing constructions of [171] and [172] are performed using a determined elliptic system provided by the conformal method, which necessarily leads to a global deformation of the initial data set, small away from the gluing site. Now, the ability of the Corvino gluing technique to establish compactly supported deformations invited the question of whether these conformal gluings could be localized. This was answered in the affirmative in [101] for CMC initial data under the additional, generically satisfied 33, assumption that there are no KIDs in a neighborhood of the gluing site.

In 105, 106, this was substantially improved upon by combining the gluing construction of [171] together with the Corvino gluing technique of [100, 113, to obtain a localized gluing construction in which the only assumption is the absence of KIDs near points. For a given $n$-manifold $M$ (which may or may not be connected) and two points $p_{a} \in M, a=1,2$, we let $\tilde{M}$ denote the manifold obtained by replacing small geodesic balls around these points by a neck $S^{n-1} \times I$. When $M$ is connected, this corresponds to performing codimension- $n$ surgery on the manifold. When the points $p_{a}$ lie in different connected components of $M$, this corresponds to taking the connected sum of those components.

Theorem 5.6 ([105, 106]). Let $(M, h, K)$ be a smooth vacuum initial data set with $M$ not necessarily connected, and consider two open sets $\Omega_{a} \subset M, a=1,2$, with compact closure and smooth boundary such that

$$
\text { the set of KIDs, } \mathscr{K}\left(\Omega_{a}\right) \text {, is trivial. }
$$

Then for all $p_{a} \in \Omega_{a}, \epsilon>0$, and $k \in \mathbb{N}$ there exists a smooth vacuum initial data set $(\tilde{M}, h(\epsilon), K(\epsilon))$ on the glued manifold $\tilde{M}$ such that $(h(\epsilon), K(\epsilon))$ is $\epsilon$-close to $(h, K)$ in a $C^{k} \times C^{k}$ topology away from $B\left(p_{1}, \epsilon\right) \cup B\left(p_{2}, \epsilon\right)$. Moreover $(h(\epsilon), K(\epsilon))$ coincides with $(h, K)$ away from $\Omega_{1} \cup \Omega_{2}$.

This result is sharp in the following sense: First note that, by the positive mass theorem, initial data for Minkowski space-time cannot locally be glued to anything which is nonsingular and vacuum. This meshes with the fact that for Minkowskian initial data, we have $\mathscr{K}(\Omega) \neq\{0\}$ for any open set $\Omega$. Next, recall that by the results in 33 , the no KIDs hypothesis in Theorem 5.6 is generically satisfied. Thus, the result can be interpreted as the statement that for generic vacuum initial data sets, the local gluing can be performed around arbitrarily chosen points $p_{a}$. In particular the collection of initial data with generic regions $\Omega_{a}$ satisfying the hypotheses of Theorem 5.6 is not empty.

The proof of Theorem [5.6 is a mixture of gluing techniques developed in [170, 171] and those of [101, 113, 115. In fact, the proof proceeds initially via a generalization of the analysis in [171] to compact manifolds with boundary. In order to have CMC initial data near the gluing points, which the analysis based on 171 requires, one makes use of the work of Bartnik [21] on the plateau problem for prescribed mean curvature spacelike hypersurfaces in a Lorentzian manifold. 
An application of the gluing techniques concerns the question of the existence of CMC slices in space-times with compact Cauchy surfaces. In [22, Bartnik showed that there exist maximally extended, globally hyperbolic solutions of the Einstein equations with dust which admit no CMC slices. Later, Eardley and Witt (unpublished) proposed a scheme for showing that similar vacuum solutions exist, but their argument was incomplete. It turns out that these ideas can be implemented using Theorem [5.6, which leads to

Corollary 5.7 (105, 106). There exist maximal globally hyperbolic vacuum spacetimes with compact Cauchy surfaces which contain no compact spacelike hypersurfaces with constant mean curvature.

\section{Evolution}

In Section 4 we have seen that solutions of the vacuum Einstein equations can be constructed by solving a Cauchy problem. It is then of interest to inquire about the global properties of the resulting space-times. A key example to keep in mind in this context is provided by the Taub-NUT metrics [228, 273, which exhibit incomplete geodesics within compact sets, closed causal curves, inequivalent extensions of maximal globally hyperbolic regions (to be defined shortly) and inequivalent conformal boundary completions at infinity. In particular they provide an example of nonuniqueness of solutions of the Cauchy problem, a problem that we address in the next section.

6.1. Strong cosmic censorship. The strong cosmic censorship (SCC) problem concerns predictability: Indeed, a fundamental requirement of physically relevant equations is that solutions should be uniquely determined by initial data. So it is important to inquire about predictability in general relativity.

In other words, we would like to know whether or not the solutions provided by Theorem 4.4 are unique. Now, it is easy to see that there can be no uniqueness unless some restrictions on the development are imposed: consider for example $(-\infty, 1) \times \mathbb{R}^{n}, \mathbb{R} \times \mathbb{R}^{n}$ and $\left(\mathbb{R} \times \mathbb{R}^{n}\right) \backslash\{(1, \overrightarrow{0})\}$ equipped with the obvious flat metric. All three space-times contain the spacelike surface $\left(\{0\} \times \mathbb{R}^{n}, \delta, 0\right)$, where $\delta$ is the Euclidean metric on $\mathbb{R}^{n}$. The first two are globally hyperbolic developments of the given initial data, but the third is not, as it is not globally hyperbolic. Obviously these are not isometric: e.g., the second is geodesically complete, while the other two are not. So to guarantee uniqueness, some further conditions are needed.

The key existence and uniqueness theorem in this context is due to ChoquetBruhat and Geroch [75] (compare [81, 95, 154). Some terminology is needed: a space-time $(\mathscr{M}, g)$ is said to be a development of an initial data set $(M, h, K)$ if there exists an embedding $i: M \rightarrow \mathscr{M}$ such that $i(M)$ is a Cauchy surface for $(\mathscr{M}, g)$, with

$$
i^{*} g=h,
$$

and with $K$ being the pull-back to $M$ of the extrinsic curvature tensor (second fundamental form) of $i(M)$. We will say that a development $(\mathscr{M}, g)$ is maximal globally hyperbolic if the following implication holds: if $\psi: \mathscr{M} \rightarrow \mathscr{M}^{\prime}$ is an isometric embedding of $\mathscr{M}$ into $\left(\mathscr{M}^{\prime}, g^{\prime}\right)$ and if $\psi(\mathscr{M}) \neq \mathscr{M}^{\prime}$, then $\mathscr{M}^{\prime}$ is not globally hyperbolic.

Note that we are not imposing any field equations on $\left(\mathscr{M}^{\prime}, g^{\prime}\right)$. One could similarly define a notion of maximality within the class of vacuum space-times, but this 
would lead to a weaker statement of the Choquet-Bruhat-Geroch theorem, which for simplicity is presented in the smooth case:

Theorem 6.1 (Existence of maximal globally hyperbolic developments [75]). For any smooth vacuum initial data $(M, h, K)$ there exists a unique, up to isometric diffeomorphism, vacuum development $(\mathscr{M}, g)$, which is inextendible in the class of smooth globally hyperbolic Lorentzian manifolds.

This theorem can be thought of as the equivalent of the usual ODE theorem of existence of maximal solutions. The generalization is, however, highly nontrivial because while the proof for ODEs deals with subsets of $\mathbb{R}$, Theorem 6.1 deals with manifolds which are dynamically obtained by patching together local solutions. The main difficulty is to prove that the patching leads to a Hausdorff topological space. The argument makes use of Lorentzian causality theory, which in turn relies heavily on $C^{2}$ differentiability of the metric. To obtain a version of Theorem 6.1 with lower differentiability, as in Theorem 4.4 or in Remark 4.2. one would need to show that the relevant parts of causal theory can be repeated in the wider setting.

While Theorem6.1 is highly satisfactory, it does not quite prove what one wants, because uniqueness is claimed in the globally hyperbolic class only. However, the Taub-NUT metrics provide an example of vacuum space-times with nonunique extensions of a maximal globally hyperbolic region. In such examples the spacetime $(\mathscr{M}, g)$ of Theorem 6.1 is unique in the class of globally hyperbolic space-times, but it can be extended in more than one way to strictly larger vacuum solutions. In such cases the extension always takes place across a Cauchy horizon, as defined in Section 2.3.3

So one cannot expect uniqueness in general. However, it has been suggested by Penrose 239] that nonuniqueness happens only in very special circumstances. The following result of Isenberg and Moncrief [173, 177, 223] (compare [160]) indicates that this might indeed be the case:

Theorem 6.2. Let $(\mathscr{M}, g)$ be a vacuum analytic space-time containing an analytic compact Cauchy horizon $\mathscr{H}$. If the null geodesics threading $\mathscr{H}$ are closed, then the Cauchy horizon is a Killing horizon; in particular, the isometry group of $(\mathscr{M}, g)$ is at least one dimensional.

The hypotheses of analyticity, compactness, and closed generators are of course highly restrictive. In any case it is conceivable that some kind of local isometries need to occur in space-times with Cauchy horizons when those conditions are not imposed; indeed, all known examples have this property. But of course existence of local isometries is a highly nongeneric property, even when vacuum equations are imposed 33], so a version of Theorem 6.2 without those undesirable hypotheses would indeed establish SCC.

Whether or not Cauchy horizons require Killing vector fields, a loose mathematical formulation of strong cosmic censorship, as formulated in 94 following Moncrief and Eardley 222] and Penrose [239], is the following:

Consider the collection of initial data for, say, vacuum or electro-vacuum space-times, with the initial data surface $M$ being compact or with asymptotically flat initial data $(M, h, K)$. For generic such data the maximal globally hyperbolic development is inextendible. 
Because of the difficulty of the strong cosmic censorship problem, a full understanding of the issues which arise in this context seems to be completely out of reach at this stage. There is therefore some interest in trying to understand that question under various restrictive hypotheses, e.g., symmetry. The simplest case, of spatially homogeneous space-times, has turned out to be surprisingly difficult, because of the intricacies of the dynamics of some of the Bianchi models discussed in Section 6.6. and has been settled in the affirmative in 111. (compare Theorem 6.8 below).

6.1.1. Gowdy toroidal metrics. The next simplest case is that of Gowdy metrics on $\mathbb{T}^{3}:=S^{1} \times S^{1} \times S^{1}$ : by definition,

(6.1) $g=e^{(\tau-\lambda) / 2}\left(-e^{-2 \tau} d \tau^{2}+d \theta^{2}\right)+e^{-\tau}\left[e^{P} d \sigma^{2}+2 e^{P} Q d \sigma d \delta+\left(e^{P} Q^{2}+e^{-P}\right) d \delta^{2}\right]$,

where $\tau \in \mathbb{R}$ and $(\theta, \sigma, \delta)$ are coordinates on $\mathbb{T}^{3}$, with the functions $P, Q$ and $\lambda$ depending only on $\tau$ and $\theta$. The metric of a maximal globally hyperbolic $U(1) \times U(1)$ symmetric vacuum space-time with $\mathbb{T}^{3}$-Cauchy surfaces can be globally written 93 . in the form (6.1) provided that the twist constants vanish:

$$
c_{a}:=\epsilon_{\alpha \beta \gamma \delta} X_{1}^{\alpha} X_{2}^{\beta} \nabla^{\gamma} X_{a}^{\delta}=0, \quad a=1,2,
$$

where the $X_{a}$ 's are the Killing vectors generating the $U(1) \times U(1)$ action. The condition $c_{1}=c_{2}=0$ is equivalent to the requirement that the family of planes $\operatorname{span}\left\{X_{1}, X_{2}\right\}^{\perp}$ is integrable.

For metrics of the form (6.1), the Einstein vacuum equations become a set of wave-map equations

$$
\begin{aligned}
P_{\tau \tau}-e^{-2 \tau} P_{\theta \theta}-e^{2 P}\left(Q_{\tau}^{2}-e^{-2 \tau} Q_{\theta}^{2}\right) & =0 \\
Q_{\tau \tau}-e^{-2 \tau} Q_{\theta \theta}+2\left(P_{\tau} Q_{\tau}-e^{-2 \tau} P_{\theta} Q_{\theta}\right) & =0
\end{aligned}
$$

which are supplemented by ODEs for the function $\lambda$,

$$
\begin{aligned}
& \lambda_{\tau}=P_{\tau}^{2}+e^{-2 \tau} P_{\theta}^{2}+e^{2 P}\left(Q_{\tau}^{2}+e^{-2 \tau} Q_{\theta}^{2}\right), \\
& \lambda_{\theta}=2\left(P_{\theta} P_{\tau}+e^{2 P} Q_{\theta} Q_{\tau}\right) .
\end{aligned}
$$

Here we write $P_{\tau}$ for $\partial_{\tau} P$, etc.

Initial data on $\mathbb{T}^{3}$ for $P$ and $Q$ have to satisfy an integral constraint,

$$
\int_{S^{1}}\left(P_{\theta} P_{\tau}+e^{2 P} Q_{\theta} Q_{\tau}\right) d \theta=0
$$

which is a consequence of 6.6 and of periodicity in $\theta$. The metric function $\lambda$ is obtained by integrating (6.5)-6.6 - Global existence of solutions to (6.3)- 6.4 was proved in 220 when the initial data are given on a hypersurface $\{\tau=$ const $\}$, and in 93 for general $U(1) \times U(1)$-symmetric Cauchy surfaces.

The question of SCC in this class of metrics has been settled by Ringström, who proved that the set of smooth initial data for Gowdy models on $\mathbb{T}^{3}$ that do not lead to the formation of Cauchy horizons contains a set which is open and dense within the set of all smooth initial data. More precisely, Ringström's main result (see [250, 251] and the references therein) is the following:

Theorem 6.3. Let $\tau_{0} \in \mathbb{R}$ and let $\mathcal{S}=\left\{\left(Q\left(\tau_{0}\right), P\left(\tau_{0}\right), Q_{\tau}\left(\tau_{0}\right), P_{\tau}\left(\tau_{0}\right)\right)\right\}$ be the set of smooth initial data for (6.3) - 6.4 satisfying (6.7). There is a subset $\mathcal{G}$ of $\mathcal{S}$ which is open with respect to the $C^{2} \times C^{1}$ topology, and dense with respect to the $C^{\infty}$ topology, such that the space-times of the form (6.1) corresponding to initial 
data in $\mathcal{G}$ are causally geodesically complete in one time direction, incomplete in the other time direction, and the Kretschmann scalar, $R_{\alpha \beta \gamma \delta} R^{\alpha \beta \gamma \delta}$, becomes unbounded in the incomplete direction of causal geodesics.

This result does indeed establish SCC in this class of metrics: to see that the resulting space-times are inextendible in the category of $C^{3}$ manifolds with $C^{2}$ Lorentzian metrics, note that the existence of any such extension would imply existence of geodesics which are incomplete in the original space-time, and along which every curvature scalar is bounded.

Theorem 6.3 is complemented by the results in 67, 104, 221, where infinitedimensional families of (nongeneric) solutions, which are extendible across a Cauchy horizon, are constructed.

The key to the understanding of the global structure of the Gowdy space-times is the analysis of the behavior of the functions $P$ and $Q$ as $\tau \rightarrow \pm \infty$. The asymptotic behavior of those functions, established by Ringström, can then be translated into statements about the behavior of the space-time geometry as those limits are approached. A central element of the proof is the existence of a velocity function

$$
v(\theta):=\lim _{\tau \rightarrow \infty} \sqrt{P_{\tau}^{2}+e^{2 P} Q_{\tau}^{2}} .
$$

Essential steps in Ringström's analysis are provided by the work on Fuchsian PDEs of Kichenassamy and Rendall [182, 245], as well as the study of the action of Geroch transformations by Rendall and Weaver [247] (compare [67]). See also [107] for the related problem of an exhaustive description of Cauchy horizons in those models.

6.1.2. Other $U(1) \times U(1)$-symmetric models. The existence of two Killing vectors is also compatible with $S^{3}, L(p, q)$ (lens spaces), and $S^{1} \times S^{2}$ topologies. Thus, to achieve a complete understanding of the set of spatially compact initial data with precisely two Killing vectors, one needs to extend Ringström's analysis to those cases. There is an additional difficulty that arises because of the occurrence of axes of symmetry, where the $(1+1)$-reduced equations have the usual singularity associated with polar coordinates. Nevertheless, in view of the analysis by Christodoulou and Tahvildar-Zadeh [90, 91] (see also [93]), the global geometry of generic maximal globally hyperbolic solutions with those topologies is reasonably well understood. This leads one to expect that one should be able to achieve a proof of SCC in those models using simple abstract arguments, but this remains to be seen.

Recall, finally, that general models with two Killing vectors $X_{1}$ and $X_{2}$ on $\mathbb{T}^{3}$ have nonvanishing twist constants 6.2. The Gowdy metrics are actually zero measure in the set of all $U(1) \times U(1)$-symmetric metrics on $\mathbb{T}^{3}$ because $c_{a} \equiv 0$ for the Gowdy models. The equations for the resulting metrics are considerably more complicated when the $c_{a}$ 's do not vanish, and only scant rigorous information is available on the global properties of the associated solutions [36, 179, 242. It seems urgent to study the dynamics of those models, as they are expected to display 37 oscillatory behavior as the singularity is approached, in the sense of Section 6.7 Thus, they should provide the simplest model in which to study this behavior.

6.1.3. Spherical symmetry. One could think that the simplest possible asymptotically flat model for studying the dynamics of the gravitational field will be obtained by requiring spherical symmetry, since then the equations should reduce to wave equations in only two variables, $t$ and $r$. Unfortunately, for vacuum space-times this turns out to be useless for this purpose because of Birkhoff's theorem 45, which 
asserts that spherically symmetric vacuum metrics are static. So, if one wishes to maintain spherical symmetry, supplementary fields are needed. The case of a scalar field was studied in a series of intricate papers over 13 years by Christodoulou, beginning with 83, and culminating in 85] with the verification of the strong cosmic censorship conjecture within the model. Christodoulou further established weak cosmic censorship in this class, an issue to which we return in the next section, and exhibited nongeneric examples for which the conclusions of these conjectures fail 84 .

The situation changes when electromagnetic fields are introduced. The analysis by Dafermos [116, 117] of the spherically symmetric Einstein-Maxwell scalar field equations yields a detailed picture of the interior of the black hole for this model, in terms of initial data specified on the event horizon and on an ingoing null hypersurface. When combined with the work by Dafermos and Rodnianski [118] on Price's law, one obtains the following global picture: initial data with a compactly supported scalar field and containing a trapped surface (see Section 7.2 below) lead to space-times which either contain a degenerate (extremal) black hole or develop a Cauchy horizon, with a space-time metric that can be continued past this horizon in a $C^{0}$ but not $C^{1}$ manner. It seems that not much is known about the properties of the degenerate solutions, which are presumably nongeneric; it would be of interest to clarify that. In any case, the work shows that strong cosmic censorship holds within the class of nondegenerate solutions with trapped surfaces, at the $C^{1}$ level, leaving behind the perplexing possibility of continuous extendability of the metric.

The reader is referred to [3, 94, 244] and the references therein for further reading on SCC.

6.2. Weak cosmic censorship. The strong cosmic censorship conjecture is an attempt to salvage predictability of Einstein's theory of gravitation. There exists a variant thereof which addresses the fact that we do not seem to observe any of the singularities that are believed to accompany gravitational collapse. The hope is then that, generically, in asymptotically flat space-times, any singular behavior that might form as a result of gravitational collapse, such as causality violations, lack of predictability, or curvature singularities, will be clothed by an event horizon. For this, one introduces the notion of future null infinity, which is an idealized boundary attached to space-time that represents, loosely speaking, the endpoints of null geodesics escaping to infinity. (In stationary situations this is closely related to the region $\mathscr{M}_{\text {ext }}$ of (3.24).) The black hole event horizon is then the boundary of the past of null infinity; compare (3.25) and (3.26). One then wishes the part of the space-time that lies outside the black hole region to be well-behaved and "sufficiently large". This is the content of the weak cosmic censorship conjecture, originally due to Penrose 239, as made precise by Christodoulou [86]: for generic asymptotically flat initial data, the maximal globally hyperbolic development has a complete future null infinity. Heuristically this means that, disregarding exceptional sets of initial data, no singularities are observed at large distances, even when the observations are continued indefinitely. One should remark that, despite the names, the strong and weak cosmic censorship conjectures are logically independent; neither follows from the other. Note also that some predictability of Einstein's theory would be salvaged if strong cosmic censorship failed with weak cosmic censorship being verified, since then the failure of predictability would be invisible to outside observers. 


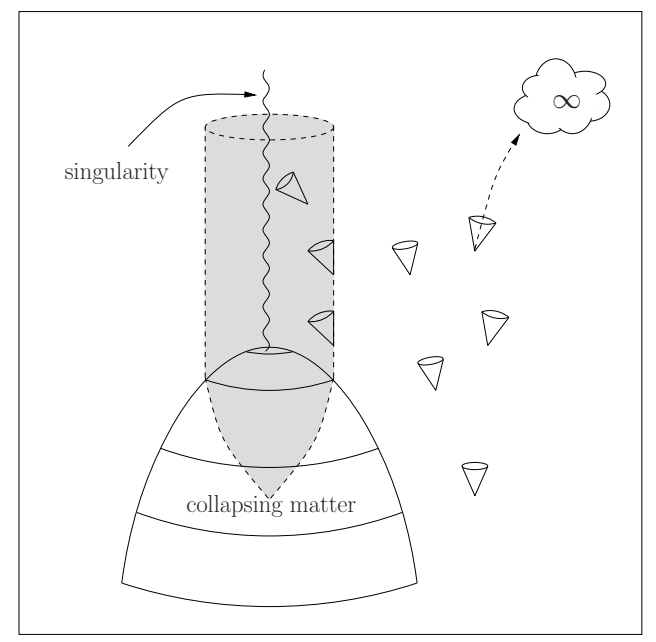

Figure 6.1. Light-cones in the Oppenheimer-Snyder collapse.

Both cosmic censorship conjectures are intimately related to the issue of gravitational collapse, the dynamical formation of black holes and singularities, first observed for a homogeneous dust model by Oppenheimer and Snyder in 1939 234, visualized in Figure 6.1.

So far the only complete analysis of weak cosmic censorship in a field-theoretical model is that of the spherically symmetric scalar field model studied by Christodoulou [84, 85, already mentioned in Section 6.1.3.

6.3. Stability of vacuum cosmological models. Not being able to understand the dynamics of all solutions, one can ask whether some features of certain particularly important solutions persist under small perturbations of initial data. For example, will geodesic completeness still hold for space-times arising from small perturbations of Minkowskian initial data? Or, will a global, all encompassing, singularity persist under perturbations of Bianchi IX initial data (see Section 6.6). Such questions are the object of stability studies.

6.3.1. $U(1)$ symmetry. Our understanding of models with exactly one Killing vector is dramatically poorer than that of $U(1) \times U(1)$-symmetric space-times. Here one only has stability results, for small perturbations within the $U(1)$ isometry class in the expanding direction ("away from the singularity"): In [70, Choquet-Bruhat considers $U(1)$-symmetric initial data $(h, K)$ for the vacuum Einstein equations on a manifold of the form $M \times S^{1}$, where $M$ is a compact surface of genus $g>1$. It is assumed that $\operatorname{tr}_{h} K$ is constant, and that $(h, K)$ are sufficiently close to $\left(h_{0}, K_{0}\right)$, where $h_{0}$ is a product metric

$$
h_{0}=\gamma+d x^{2},
$$

with $\gamma$ being a metric of constant Gauss curvature on $M$, and with $K_{0}$ proportional to $h_{0}$. The sign of the trace of $K_{0}$ determines an expanding time direction and a contracting one. Under those conditions, Choquet-Bruhat proves that the solution exists for an infinite proper time in the expanding direction. The analysis builds upon previous work by Choquet-Bruhat and Moncrief [80, where a supplementary 
polarization condition has been imposed. Not much is known in the contracting direction in the $U(1)$-symmetric models (see, however, [176]), where mixmaster behavion 8 is expected [35, 39]; compare [40].

6.3.2. Future stability of hyperbolic models. The proof of the above result bears some similarity to the future stability theorem of Andersson and Moncrief [13, as generalized in [11, for spatially compact hyperbolic models without any symmetries. Those authors consider initial data near a negatively curved compact space form, with the extrinsic curvature being close to a multiple of the metric, obtaining future geodesic completeness in the expanding direction. The control of the solution is obtained by studying the Bel-Robinson tensor and its higher-derivatives analogues. A striking ingredient of the proof is an elliptic-hyperbolic system of equations, used to obtain local existence in time [12].

\subsection{Stability of Minkowski space-time.}

6.4.1. The Christodoulou-Klainerman proof. One of the flagship results in mathematical general relativity is nonlinear stability of Minkowski space-time, first proved by Christodoulou and Klainerman [88. One starts with an asymptotically flat vacuum initial data set $(h, K)$ on $\mathbb{R}^{3}$. Under standard asymptotic flatness conditions, for $(h, K)$ sufficiently close to Minkowskian data, the maximal globally hyperbolic development $(\mathscr{M}, g)$ of the data contains a maximal hypersurface, i.e., a hypersurface satisfying $\operatorname{tr}_{h} K=0$; this follows from the results in [19, 25, 89. So without loss of generality one can, in the small data context, assume that the initial data set is maximal.

The precise notion of smallness needed for the Christodoulou-Klainerman theorem is defined as follows: For $p \in \Sigma \approx \mathbb{R}^{3}, a>0$, consider the quantity

$$
Q(a, p)=a^{-1} \int_{\Sigma}\left\{\sum_{\ell=0}^{1}\left(d_{p}^{2}+a^{2}\right)^{\ell+1}\left|\nabla^{\ell} \operatorname{Ric}\right|^{2}+\sum_{\ell=1}^{2}\left(d_{p}^{2}+a^{2}\right)^{\ell}\left|\nabla^{\ell} K\right|^{2}\right\} d \mu_{g},
$$

where $d_{p}$ is the geodesic distance function from $p$, Ric is the Ricci tensor of the metric $g, d \mu_{g}$ is the Riemannian measure of the metric $g$, and $\nabla$ is the Riemannian connection of $g$. Let

$$
Q_{*}=\inf _{a>0, p \in \Sigma} Q(a, p) .
$$

Christodoulou and Klainerman prove causal geodesic completeness of $(\mathscr{M}, g)$ provided that $Q_{*}$ is sufficiently small. The proof proceeds via an extremely involved bootstrap argument involving a foliation by maximal hypersurfaces $\Sigma_{t}$ together with an analysis of the properties of an optical function $u$. In the context here this is a solution of the eikonal equation

$$
g^{\alpha \beta} \partial_{a} u \partial_{\beta} u=0
$$

the level sets $C_{u}$ of which intersect $\Sigma_{t}$ in spheres which expand as $t$ increases. We have:

Theorem 6.4 (Global stability of Minkowski space-time). Assume that $(M, h, K)$ is maximal, with 9

$$
h_{i j}=\delta_{i j}+o_{3}\left(r^{-1 / 2}\right), \quad K_{i j}=o_{2}\left(r^{-3 / 2}\right) .
$$

\footnotetext{
${ }^{8}$ See the discussion after Theorem 6.7 and Section 6.7

${ }^{9} \mathrm{~A}$ function $f$ on $M$ is $o_{k}\left(r^{-\lambda}\right)$ if $r^{\lambda+i} \nabla^{i} f \rightarrow 0$ as $r \rightarrow \infty$ for all $i=0, \ldots, k$.
} 
There is an $\epsilon>0$ such that if $Q_{*}<\epsilon$, then the maximal globally hyperbolic development $(\mathscr{M}, g)$ of $(M, h, K)$ is geodesically complete.

The above version of Theorem 6.4 is due to Bieri [44. The original formulation in 88. assumes moreover that

$$
h=(1+2 M / r) \delta+o_{4}\left(r^{-3 / 2}\right), \quad K=o_{3}\left(r^{-5 / 2}\right),
$$

and in the definition (6.8) a term involving $K$ with $\ell=0$ is added.

By definition, asymptotically flat initial data sets approach the Minkowskian ones as one recedes to infinity. One therefore expects that at sufficiently large distances, one should obtain global existence, in the sense that the maximal globally hyperbolic development contains complete outgoing null geodesics. This question has been addressed by Klainerman and Nicolò [185, 186, 187]; the reader is referred to those references for precise statements of the hypotheses made:

Theorem 6.5. Consider an asymptotically flat initial data set $(M, h, K)$ with maximal globally hyperbolic development $(\mathscr{M}, g)$. Let $\Omega_{r}$ denote a conditionally compact domain bounded by a coordinate sphere $S_{r} \subset \mathscr{M}_{\mathrm{ext}}$. There exists $R>0$ such that for all $r \geq R$ the generators of the boundary $\partial J^{+}\left(\Omega_{r}\right)$ of the domain of influence $J^{+}\left(\Omega_{r}\right)$ of $\Omega_{r}$ are future-complete.

Both in 88 and in 186 one can find detailed information concerning the behavior of null hypersurfaces as well as the rate at which various components of the Riemann curvature tensor approach zero along timelike and null geodesics.

6.4.2. The Lindblad-Rodnianski proof. A completely new proof of stability of Minkowski space-time has been given by Lindblad and Rodnianski [200, 201. The method provides less detailed asymptotic information than [88] and [186] on various quantities of interest but is much simpler. The argument is flexible enough to allow the inclusion of a scalar field or of a Maxwell field [206, 207] (compare [44 for an analysis along the lines of the Christodoulou-Klainerman approach), and generalizes to higher dimensions [73. Further it allows the following, less restrictive than that in [88, 186], asymptotic behavior of the initial data, for some $\alpha>0$ :

$$
h=(1+2 m / r) \delta+O\left(r^{-1-\alpha}\right), \quad K=O\left(r^{-2-\alpha}\right) .
$$

Lindblad and Rodnianski consider the Einstein-Maxwell equations with a neutral scalar field:

$$
R_{\mu \nu}-\frac{R}{2} g_{\mu \nu}=T_{\mu \nu}+\hat{T}_{\mu \nu}
$$

with

$$
\hat{T}_{\mu \nu}=\partial_{\mu} \psi \partial_{\nu} \psi-\frac{1}{2} g_{\mu \nu}\left(g^{\alpha \beta} \partial_{\alpha} \psi \partial_{\beta} \psi\right), \quad T_{\mu \nu}=2\left(F_{\mu \lambda} F_{\nu}{ }^{\lambda}-\frac{1}{4} g_{\mu \nu} F^{\lambda \rho} F_{\lambda \rho}\right) .
$$

The initial data are prescribed on $\mathbb{R}^{n}$, so that the Maxwell field $F$ has a global potential $A, F_{\mu \nu}=\partial_{\mu} A_{\nu}-\partial_{\nu} A_{\mu}$. The matter field equations read

$$
D_{\mu} F^{\mu \nu}=0, \quad \square_{g} \psi=0 .
$$

The initial data, denoted by $\left(\stackrel{\circ}{h}, \stackrel{\circ}{K}, \AA\right.$ A $, \stackrel{\circ}{E}, \psi_{0}, \psi_{1}$ ) (where, roughly speaking, $\AA$ is the initial value for the Maxwell potential and $E^{\circ}$ is the initial value for the electric field) 
satisfy the following asymptotic conditions, for $r=|x| \rightarrow \infty$, with some $\alpha>0$ :

$$
\begin{aligned}
& \stackrel{\circ}{h}_{i j}=\left\{\begin{array}{l}
\left(1+\frac{2 m}{r}\right) \delta_{i j}+O\left(r^{-1-\alpha}\right), \text { for } n=3, \\
\delta_{i j}+O\left(r^{\frac{1-n}{2}-\alpha}\right), \text { for } n \geq 4,
\end{array}\right. \\
& \stackrel{\AA}{A}=O\left(r^{\frac{1-n}{2}-\alpha}\right), \quad \stackrel{\circ}{K}_{i j}=O\left(r^{-\frac{n+1}{2}-\alpha}\right), \quad \stackrel{\circ}{E}=O\left(r^{-\frac{n+1}{2}-\alpha}\right), \\
& \psi_{0}:=\left.\psi\right|_{t=0}=O\left(r^{\frac{1-n}{2}-\alpha}\right), \quad \psi_{1}:=\left.\partial_{t} \psi\right|_{t=0}=O\left(r^{-\frac{n+1}{2}-\alpha}\right) .
\end{aligned}
$$

The strategy is to impose globally the wave coordinates condition

$$
\partial_{\mu}\left(g^{\mu \nu} \sqrt{|\operatorname{det} g|}\right)=0 \quad \forall \nu=0, \ldots, n,
$$

as well as the Lorenz gauge for the electromagnetic potential $A_{\mu}$,

$$
\partial_{\mu}\left(\sqrt{|\operatorname{det} g|} A^{\mu}\right)=0 .
$$

The initial data are decomposed as

$$
h_{\mu \nu}^{1}=h_{\mu \nu}-h_{\mu \nu}^{0}, \quad \text { with } h_{\mu \nu}^{0}(t)=\left\{\begin{array}{l}
\chi(r / t) \chi(r) \frac{2 m}{r} \delta_{\mu \nu} \quad \text { for } n=3, \\
0 \text { for } n \geq 4,
\end{array}\right.
$$

where $\chi \in C^{\infty}$ is any function such that $\chi(s)$ equals 1 for $s \geq 3 / 4$ and 0 for $s \leq 1 / 2$. The proof relies heavily on the structure of the nonlinear terms in wave coordinates.

Recall that there exists an extensive literature on wave equations in $3+1$ dimensions with nonlinearities satisfying the null condition [183, 184, but the nonlinearities that arise do not satisfy that condition. The argument works only because different components of $h$ can be treated on a different footing. Indeed, for solutions of the wave equation on Minkowski space-time, the derivatives in directions tangent to the light-cones decay faster than the transverse ones. But the wave coordinates condition (6.15) can be used to express the transverse derivatives of some components of $g_{\mu \nu}$ in terms of tangential derivatives of the remaining ones. This provides control of the nonlinearities.

We also note the small data global existence results of [166, 195] on $\mathbb{R}^{n+1}, n \geq 4$, and of 82 for odd $n \geq 5$. The structure conditions there are general enough to cover the Einstein equations in wave coordinates, but the assumptions on the fall-off of initial data exclude nontrivial solutions of the vacuum constraint equations 10

We have:

Theorem 6.6. Consider smooth initial data $\left(\stackrel{\circ}{h}, \stackrel{\circ}{K}, \stackrel{\circ}{A}, \stackrel{\circ}{E}, \psi_{0}, \psi_{1}\right)$ on $\mathbb{R}^{n}, n \geq 3$, satisfying (6.14) together with the Einstein-Maxwell constraint equations. Let $N \in$ $\mathbb{N}$, suppose that $N_{n}:=N+\left[\frac{n+2}{2}\right]-2 \geq 6+2\left[\frac{n+2}{2}\right]$, and set

$$
\begin{aligned}
E_{N_{n}, \gamma}(0)= & \sum_{0 \leq|I| \leq N_{n}}\left(\left\|(1+r)^{1 / 2+\gamma+|I|} \nabla \nabla^{I} h_{0}^{1}\right\|_{L^{2}}^{2}+\left\|(1+r)^{1 / 2+\gamma+|I|} \nabla^{I} \stackrel{\circ}{K}\right\|_{L^{2}}^{2}\right. \\
& +\left\|(1+r)^{1 / 2+\gamma+|I|} \nabla \nabla^{I} \stackrel{\circ}{A}\right\|_{L^{2}}^{2}+\left\|(1+r)^{1 / 2+\gamma+|I|} \nabla^{I} \stackrel{\circ}{E}\right\|_{L^{2}}^{2} \\
& \left.+\left\|(1+r)^{1 / 2+\gamma+|I|} \nabla \nabla^{I} \psi_{0}\right\|_{L^{2}}+\left\|(1+r)^{1 / 2+\gamma+|I|} \nabla^{I} \psi_{1}\right\|_{L^{2}}\right) .
\end{aligned}
$$

\footnotetext{
${ }^{10}$ In [166, 195], compactly supported data are considered. In the theorem for general quasilinear systems given in 82 the initial data are in a Sobolev space which requires fall-off at infinity faster than $r^{-n-3 / 2}$. In both cases the positive energy theorem implies that such initial data lead to Minkowski space-time.
} 
Let $m$ be the ADM mass of $\stackrel{\circ}{h}$. For every $\gamma_{0}>0$ there exists $\varepsilon_{0}>0$, with $\gamma_{0}\left(\varepsilon_{0}\right) \rightarrow 0$ as $\varepsilon_{0} \rightarrow 0$, such that if

$$
\sqrt{E_{N_{n}, \gamma}(0)}+m \leq \varepsilon_{0}
$$

for some $\gamma>\gamma_{0}$, then the maximal globally hyperbolic development of the initial data is geodesically complete.

6.5. Toward stability of Kerr: Wave equations on black hole backgrounds. Since the pioneering work of Christodoulou and Klainerman on stability of Minkowski space-time, many researchers have been looking into ways to address the question of stability of Kerr black holes. The first naive guess would be to study stability of Schwarzschild black holes, but those cannot be stable since a generic small perturbation will introduce angular momentum. The current strategy is to study, as a first step, linear wave equations on black hole backgrounds, with the hope that sufficiently robust linear decay estimates can be bootstrapped to produce a nonlinear stability proof. Due to limited space we will not review those results, referring the reader to recent important papers on the subject [4, 46, 47, 119, 120, 121, 210, 271, 272, 128; ; see also [138] and the references therein.

6.6. Bianchi $A$ metrics. Another important example of the intricate dynamical behavior of solutions of the Einstein equations is provided by the Bianchi $A$ vacuum metrics. The key insight provided by these space-times is the supposedly chaotic behavior of large families of metrics in this class when a singularity is approached. This dynamics has been conjectured to be generic; we will return to this issue in Section 6.7. As will be seen shortly, in Bianchi $A$ space-times, the Einstein evolution equations reduce to a polynomial dynamical system on an algebraic fourdimensional submanifold of $\mathbb{R}^{5}$. The spatial parts of the Bianchi geometries provide a realization of six, out of eight, homogeneous geometries in three dimensions which form the basis of Thurston's geometrization program.

For our purposes here we define the Bianchi space-times as maximal globally hyperbolic vacuum developments of initial data which are invariant under a simply transitive group of isometries. Here the transitivity of the isometry group is meant at the level of initial data, and not for the space-time. The name is a tribute to Bianchi, who gave the classification of three-dimensional Lie algebras which underline the geometry here. These metrics split into two classes, Bianchi $A$ and Bianchi $B$, as follows. Let $G$ be a three-dimensional Lie group, and let $Z_{i}, i=1,2,3$, denote a basis of left-invariant vector fields on $G$. Define the structure constants $\gamma_{i j}^{k}$ by the formula

$$
\left[Z_{i}, Z_{j}\right]=\gamma_{i j}{ }^{k} Z_{k}
$$

The Lie algebra and Lie group are said to be of class $A$ if $\gamma_{i k}{ }^{k}=0$; class $B$ are the remaining ones. The classes $A$ and $B$ correspond in mathematical terminology to the unimodular and nonunimodular Lie algebras. A convenient parameterization of the structure constants is provided by the symmetric matrix $n^{i j}$ defined as

$$
n^{i j}=\frac{1}{2} \gamma_{k l}{ }^{(i} \epsilon^{j) k l} \text {. }
$$

This implies that $\gamma_{i j}{ }^{k}=\epsilon_{i j m} n^{k m}$. The Bianchi $A$ metrics are then divided into six classes, according to the eigenvalues of the matrix $n^{i j}$, as described in Table 6.1 For the Bianchi IX metrics, of particular interest to us here, the group $G$ is $S U(2)$. 
TABLE 6.1. Lie groups of Bianchi class A.

\begin{tabular}{ccccl}
\hline Bianchi type & $n_{1}$ & $n_{2}$ & $n_{3}$ & Simply connected group \\
\hline I & 0 & 0 & 0 & Abelian $\mathbb{R}^{3}$ \\
II & + & 0 & 0 & Heisenberg \\
VI $_{0}$ & 0 & + & - & Sol (isometries of the Minkowski plane $\mathbb{R}^{1,1}$ ) \\
VII $_{0}$ & 0 & + & + & universal cover of Euclid (isometries of $\mathbb{R}^{2}$ ) \\
VIII & - & + & + & universal cover of $S L(2, \mathbb{R})$ \\
IX & + & + & + & $S U(2)$ \\
\hline
\end{tabular}

Let $G$ be any three-dimensional Lie group, the Lie algebra of which belongs to the Bianchi $A$ class. (The $G$ 's are closely related to the Thurston geometries; see Table 6.1 and compare [3, Table 2].) Denote by $\left\{\sigma^{i}\right\}$ the basis dual to $\left\{Z_{i}\right\}$. It is not too difficult to show that both $A$ and $B$ Bianchi metrics can be globally written as

$$
g=-d t^{2}+h_{i j}(t) \sigma^{i} \sigma^{j}, \quad t \in I,
$$

with a maximal time interval $I$.

There are various ways to write the Einstein equations for a metric of the form (6.21). We use the formalism introduced by Wainwright and Hsu [276], which has proven to be most useful for analytical purposes [243, 248, 249, and we follow the presentation in 248]. Let

$$
\sigma_{i j}=K_{i j}-\frac{1}{3} \operatorname{tr}_{h} K h_{i j}, \quad \theta:=\operatorname{tr}_{h} K,
$$

be the trace-free part of the extrinsic curvature tensor of the level sets of $t$. Away from the (isolated) points at which $\theta$ vanishes, one can introduce

$$
\begin{aligned}
\Sigma_{i j} & =\sigma_{i j} / \theta, \\
N_{i j} & =n_{i j} / \theta, \\
B_{i j} & =2 N_{i}{ }^{k} N_{k j}-N_{k}^{k} N_{i j}, \\
S_{i j} & =B_{i j}-\frac{1}{3} B_{k}^{k} \delta_{i j} .
\end{aligned}
$$

Set $\Sigma_{+}=\frac{3}{2}\left(\Sigma_{22}+\Sigma_{33}\right)$ and $\Sigma_{-}=\sqrt{3}\left(\Sigma_{22}-\Sigma_{33}\right) / 2$. If we let $N_{i}$ be the eigenvalues of $N_{i j}$, the vacuum Einstein equations (a detailed derivation of which can be found in 248) lead to the following autonomous, polynomial dynamical system:

$$
\begin{aligned}
& N_{1}^{\prime}=\left(q-4 \Sigma_{+}\right) N_{1}, \\
& N_{2}^{\prime}=\left(q+2 \Sigma_{+}+2 \sqrt{3} \Sigma_{-}\right) N_{2}, \\
& N_{3}^{\prime}=\left(q+2 \Sigma_{+}-2 \sqrt{3} \Sigma_{-}\right) N_{3}, \\
& \Sigma_{+}^{\prime}=-(2-q) \Sigma_{+}-3 S_{+}, \\
& \Sigma_{-}^{\prime}=-(2-q) \Sigma_{-}-3 S_{-},
\end{aligned}
$$

where a prime denotes derivation with respect to a new time coordinate $\tau$ defined by

$$
\frac{d t}{d \tau}=\frac{3}{\theta}
$$


Further,

$$
\begin{aligned}
q & =2\left(\Sigma_{+}^{2}+\Sigma_{-}^{2}\right) \\
S_{+} & =\frac{1}{2}\left[\left(N_{2}-N_{3}\right)^{2}-N_{1}\left(2 N_{1}-N_{2}-N_{3}\right)\right], \\
S_{-} & =\frac{\sqrt{3}}{2}\left(N_{3}-N_{2}\right)\left(N_{1}-N_{2}-N_{3}\right) .
\end{aligned}
$$

The vacuum constraint equations reduce to one equation,

$$
\Sigma_{+}^{2}+\Sigma_{-}^{2}+\frac{3}{4}\left[N_{1}^{2}+N_{2}^{2}+N_{3}^{2}-2\left(N_{1} N_{2}+N_{2} N_{3}+N_{3} N_{1}\right)\right]=1 .
$$

The points $\left(N_{1}, N_{2}, N_{3}, \Sigma_{+}, \Sigma_{-}\right)$can be classified according to the values of $N_{1}, N_{2}, N_{3}$ in the same way as the $n_{i}$ 's in Table 6.1. The sets $N_{i}>0, N_{i}<0$, and $N_{i}=0$ are invariant under the flow determined by (6.22), and one can therefore classify solutions to (6.22) - 6.25) accordingly. Bianchi IX solutions correspond, up to symmetries of the system, to points with all $N_{i}$ 's positive, while for Bianchi VIII solutions one can assume that two $N_{i}$ 's are positive and the third is negative.

Points with $N_{1}=N_{2}=N_{3}=0$ correspond to Bianchi I models. The associated vacuum metrics were first derived by Kasner and take the form

$$
d s^{2}=-d t^{2}+\sum_{i=1}^{3} t^{2 p_{i}} d x^{i} \otimes d x^{i}, \quad p_{1}+p_{2}+p_{3}=p_{1}^{2}+p_{2}^{2}+p_{3}^{2}=1 .
$$

An important role in the analysis of (6.22) is played by the Kasner circle, defined as the set $\{q=2\}$. These points belong to the configuration space, as determined by (6.25), for Bianchi I models, but the equation $q=2$ is incompatible with 6.25) for Bianchi IX metrics. Nevertheless, we shall see shortly that the Kasner circle plays an essential role in the analysis of the Bianchi IX dynamics.

The set $\Sigma_{-}=0, N_{2}=N_{3}$, together with its permutations, is invariant under the flow of 6.22 - 6.25). In the Bianchi IX case, these are the Taub solutions. In the Bianchi VIII case, the corresponding explicit solutions, known as the NUT metrics, have been found by Newman, Tamburino and Unti 228, and they exhibit properties similar to the Bianchi IX Taub solutions.

The $\omega$-limit of an orbit $\gamma$ of a dynamical system is defined as the set of accumulation points of that orbit. In [248, 249, Ringström proves the following:

Theorem 6.7. The $\omega$-limit set of each non-NUT Bianchi VIII orbit contains at least two distinct points on the Kasner circle. Similarly, non-Taub-NUT Bianchi IX orbits have at least three distinct $\omega$-limit points on the Kasner circle.

The picture which emerges from a numerical analysis of 6.22) (see 38, 112 and the references therein) is the following: Every non-Taub-NUT Bianchi IX orbit approaches some point on the Kasner circle; there it performs a "bounce", after which it eventually approaches another point on the Kasner circle, and so on. Theorem 6.7 establishes the validity of this picture. The numerical analysis further suggests that generic orbits will have a dense $\omega$-limit set on the Kasner circle; this is compatible with, but does not follow from, Ringström's analysis. It has been argued that the map which associates to each bounce the nearest point on the Kasner circle possesses chaotic features; this is at the origin of the mixmaster behavior terminology, sometimes used in this context. Major progress concerning this issue has been achieved in [199, 28, 246], where existence of orbits exhibiting 


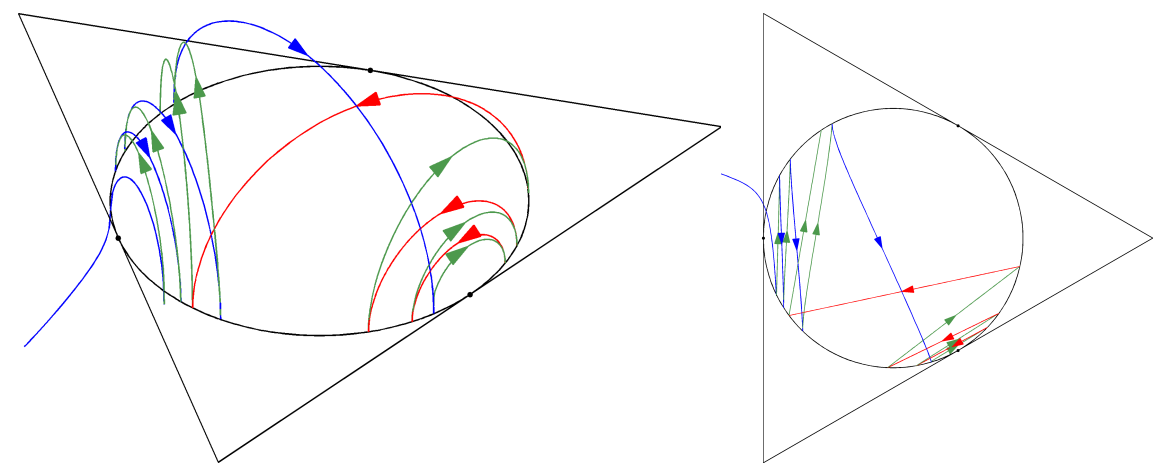

Figure 6.2. A few "bounces" in a typical Bianchi IX orbit; figures and numerics by Woei-Chet Lim. The vertical axis represents $N_{1}$ (red), $N_{2}$ (green), $N_{3}$ (blue), with only the biggest of the $N_{i}$ 's plotted. The Kasner circle and the triangle for the Kasner billiard in the $\left(\Sigma_{+}, \Sigma_{-}\right)$-plane are shown. The projected trajectories can be seen to approach the billiard ones.

the above behavior has been established, but the question of what happens for all, or for generic, orbits remains open.

The following result of Ringström [248] provides further insight into the geometry of Bianchi IX space-times:

Theorem 6.8. In all maximal globally hyperbolic developments $(\mathscr{M}, g)$ of nonTaub-NUT Bianchi IX vacuum initial data or of non-NUT Bianchi VIII vacuum initial data, the Kretschmann scalar

$$
R_{\alpha \beta \gamma \delta} R^{\alpha \beta \gamma \delta}
$$

is unbounded along inextendible causal geodesics.

Note that the observation of curvature blow-up provides a proof, alternative to that of [111], of the nonexistence of Cauchy horizons in generic Bianchi IX models.

We close this section by mentioning that no similar rigorous results are known concerning the global dynamical properties of Bianchi models of class $B$; compare [159.

6.7. The mixmaster conjecture. The most important question in the study of the Cauchy problem is that of the global properties of the resulting space-times. So far we have seen examples of geodesically complete solutions (e.g., small perturbations of Minkowski space-time), or all-encompassing singularities (e.g., generic Bianchi models), or of Cauchy horizons (e.g., Taub-NUT metrics). The geodesically complete solutions are satisfying but dynamically uninteresting, while the strong cosmic censorship conjecture expresses the hope that Cauchy horizons will almost never occur. So it appears essential to have a good understanding of the remaining cases, presumably corresponding to singularities. Belinski, Khalatnikov and Lifschitz [35] suggested that, near singularities, at each space point the dynamics of the gravitational field resembles that of generic Bianchi metrics, as described in Section 6.6. Whether or not this is true, and in which sense, remains to be seen; in any case the idea, known as the BKL conjecture, provided guidance (and still does) to 
a significant body of research on general relativistic singularities; see [18, 124, 129] and the references therein. This then leads to the mathematical challenge of making sense of the associated slogan, namely that the singularity in generic gravitational collapse is spacelike, local, and oscillatory. Here spacelike is supposed to mean that strong cosmic censorship holds. The term local refers to the idea that, near generic singularities, there should exist coordinate systems in which the metric asymptotes are to a solution of equations in which spatial derivatives of appropriately chosen fields have been neglected 11 Finally, oscillatory is supposed to convey the idea that the approximate solutions will actually be provided by the Bianchi IX metrics.

The main rigorous evidence for a relatively large class of vacuum 12 space-times with singularities which are spacelike and local in the sense described above is Ringström's Theorem 6.3, describing generic Gowdy metrics, but the resulting singularities are not oscillatory. This is not in contradiction with the conjecture, since the Gowdy metrics are certainly not generic, whether in the space of all metrics or in the space of $U(1) \times U(1)$-symmetric ones. (As mentioned in Section 6.3.1, generic $U(1) \times U(1)$ metrics have nonvanishing twist constants $c_{a}$ as defined by (6.2).) The numerical studies of [37] suggest that the switching-on of the twist constants will indeed generically lead to some kind of oscillatory behavior.

In fact, BKL put emphasis on Bianchi IX models, while some other authors seem to favor Bianchi $\mathrm{VI}_{-1 / 9}$, or not-necessarily Bianchi, oscillations [35, 39, 124, 156. 275. Moreover, it has been suggested that the oscillatory behavior disappears in space-time dimensions higher than 10 [124, 126], and large families of nonoscillatory solutions with singularities have indeed been constructed in [125]. This leads naturally to the following, somewhat loose, conjecture:

Conjecture 6.9 (Mixmaster conjecture). Let $n+1 \leq 10$. There exist open sets of vacuum metrics for which some natural geometric variables undergo oscillations of increasing complexity along inextendible geodesics of unbounded curvature.

The BKL conjecture would thus be a more precise version of the above, claiming moreover genericity of the behavior and pointing to the Bianchi dynamics as the right model. Those properties are so speculative that we decided not to include them in Conjecture 6.9.

The only examples so far of oscillatory singularities which are not spatially homogeneous have been constructed by Berger and Moncrief [40. There, a solutiongenerating transformation is applied to Bianchi IX metrics, resulting in nonhomogeneous solutions governed by the oscillatory functions arising from a non-Taub Bianchi IX metric. The resulting metrics have at least one but not more than two Killing vectors. The analysis complements the numerical evidence for oscillatory behavior in $U(1)$-symmetric models presented in [41].

\footnotetext{
${ }^{11}$ The resulting truncated equations should then presumably resemble the equations satisfied by spatially homogeneous metrics. However, different choices of quantities which are expected to be time-independent will lead to different choices of the associated notion of homogeneity. For instance, in 35 the Bianchi VIII and IX types are singled out, and the notion of genericity of those types within the Bianchi $A$ class is read from Table 6.1 as: "something that can be non-zero is more generic than something that is". On the other hand, the analysis in [156] seems to single out Bianchi VI-1/9 metrics.

${ }^{12}$ See, however, 14 125 for a class of space-times with sources; 125] also covers vacuum in space dimensions $n \geq 10$.
} 


\section{Marginally trapped SURFACES}

There have been some interesting recent developments at the interface of spacetime geometry and the theory of black holes associated with the notion of marginally outer trapped surfaces. Let $\Sigma$ be a codimension-two spacelike submanifold of a space-time $\mathscr{M}$. Under suitable orientation assumptions, there exist two families of future-directed null geodesics issuing orthogonally from $\Sigma$. If one of the families has vanishing expansion along $\Sigma$, then $\Sigma$ is called a marginally outer trapped surface (or an apparent horizon). The notion of a marginally outer trapped surface was introduced early in the development of the theory of black holes, as the occurrence of the former signals the presence of the latter. More recently, marginally outer trapped surfaces have played a fundamental role in quasi-local descriptions of black holes and have been useful in numerical simulations of black hole space-times; see, e.g., 17. Marginally outer trapped surfaces arose in a more purely mathematical context in the work of Schoen and Yau [260] concerning the existence of solutions to the Jang equation, in connection with their proof of the positivity of mass.

Mathematically, marginally outer trapped surfaces may be viewed as space-time analogues of minimal surfaces in Riemannian manifolds. Despite the absence of a variational characterization such as that for minimal surfaces 13 marginally trapped surfaces have recently been shown to satisfy a number of analogous properties; cf., in particular, 17, 8, 9, 10, 16, 131, 145] and the recent review [5. The aim of this section is to describe some of these mathematical developments.

7.1. Null hypersurfaces. Each family of null geodesics issuing orthogonally from $\Sigma$, as described above, forms a smooth null hypersurface near $\Sigma$. It would be useful at this stage to discuss some general aspects of such hypersurfaces. Null hypersurfaces have an interesting geometry and play an important role in general relativity. In particular, as we have seen, they represent horizons of various sorts, such as the event horizons discussed in Section 3 .

Let $\left(\mathscr{M}^{n+1}, g\right)$ be a space-time, with $n \geq 2$. A smooth null hypersurface in $\mathscr{M}$ is a smooth codimension-one submanifold $\mathscr{N}=\mathscr{N}^{n}$ of $\mathscr{M}$ such that the restriction of $g$ to each tangent space $T_{p} \mathscr{N}$ of $\mathscr{N}$ is degenerate. This, together with the Lorentz signature, implies that there is a unique direction of degeneracy in each tangent space $T_{p} \mathscr{N}$. Thus, every null hypersurface $\mathscr{N}$ comes equipped with a smooth future-directed null vector field $K(g(K, K)=0)$ defined on, and tangent to, $\mathscr{N}$ such that the normal space of $K$ at each $p \in \mathscr{N}$ coincides with the tangent space of $\mathscr{N}$ at $p$, i.e., $K_{p}^{\perp}=T_{p} \mathscr{N}$ for all $p \in \mathscr{N}$. Tangent vectors to $\mathscr{N}$, transverse to $K$, are then necessarily spacelike. The null vector field $K$ associated to $\mathscr{N}$ is unique up to positive pointwise rescaling. However, there is, in general, no canonical way to set the scaling.

Two simple examples arise in Minkowski space $\mathbb{R}^{1, n}$. The past and future cones $\partial I^{-}(p)$ and $\partial I^{+}(p)$ are smooth null hypersurfaces away from the vertex $p$. Each nonzero null vector $v \in T_{p} \mathbb{R}^{1, n}$ determines a null hyperplane $\Pi=\left\{q \in \mathbb{R}^{1, n}\right.$ : $\eta(\overrightarrow{p q}, v)=0\}$, where $\eta$ is the Minkowski metric, and $\overrightarrow{p q}$ is the tangent vector at $p$ representing the displacement from $p$ to $q$.

\footnotetext{
${ }^{13}$ There seems to be no analogue of the area functional.
} 
It is a fundamental fact that the integral curves of $K$ are null geodesics, though perhaps not affinely parameterized; this will depend on the scaling of $K$. Thus $\mathscr{N}$ is ruled by null geodesics, called the null generators of $\mathscr{N}$. For example the future cone $\mathscr{N}=\partial I^{+}(p) \backslash\{p\}$ in Minkowski space is ruled by future-directed null rays emanating from $p$.

The null expansion scalar $\theta$ of $\mathscr{N}$ with respect to $K$ is a smooth function on $\mathscr{N}$ that gives a measure of the average expansion of the null generators of $\mathscr{N}$ towards the future. In essence, $\theta$ is defined as the divergence of the vector field $K$ along $\mathscr{N}$. To be precise, given $p \in \mathscr{N}$, let $\Pi_{n-1}$ be a codimension-one subspace of $T_{p} \mathscr{N}^{n}$ transverse to $K_{p}$. The metric $g$, restricted to $\Pi_{n-1}$, will be positive definite. Let $\left\{e_{1}, e_{2}, \ldots, e_{n-1}\right\}$ be an orthonormal basis for $\Pi_{n-1}$ with respect to $g$. Then $\theta$ at $p$ is defined as

$$
\theta(p)=\sum_{i=1}^{n-1} g\left(\nabla_{e_{i}} K, e_{i}\right)
$$

Interestingly, due to the fact that $K$ is null, this value is independent of the choice of transverse subspace $\Pi_{n-1}$ as well as of the choice of an orthonormal basis for $\Pi_{n-1}$, and so the expansion scalar $\theta$ is well defined.

While $\theta$ depends on the choice of $K$, it does so in a simple way. As easily follows from equation (7.1), a positive rescaling of $K$ rescales $\theta$ in the same way: if $\tilde{K}=f K$, then $\tilde{\theta}=f \theta$. Thus the sign of the null expansion $\theta$ does not depend on the scaling of $K: \theta>0$ means expansion on average of the null generators, and $\theta<0$ means contraction on average. In Minkowski space, the future null cone $\mathscr{N}=\partial I^{+}(p) \backslash\{p\}$ has $\theta>0$, and the past cone $\mathscr{N}=\partial I^{-}(p) \backslash\{p\}$ has $\theta<0$.

It is useful to understand how the null expansion varies as one moves along a null generator of $\mathscr{N}$. Let $s \rightarrow \eta(s)$ be a null geodesic generator of $\mathscr{N}$, and assume that $K$ is scaled so that $\eta$ is affinely parameterized. Then it can be shown that the null expansion scalar $\theta=\theta(s)$ along $\eta$ satisfies the propagation equation

$$
\frac{d \theta}{d s}=-\operatorname{Ric}\left(\eta^{\prime}, \eta^{\prime}\right)-\sigma^{2}-\frac{1}{n-1} \theta^{2}
$$

where $\sigma \geq 0$, the shear scalar, measures the deviation from perfect isotropic expansion. Equation (7.2) is known in the relativity community as the Raychaudhuri equation (for a null geodesic congruence) [154], and, together with a timelike version, plays an important role in the proofs of the classical Hawking-Penrose singularity theorems 154]. There are well-known Riemannian counterparts to this equation, going back to work of Calabi 63.

Equation (7.2) shows how the curvature of space-time influences the expansion of the null generators. We consider here a simple application of the Raychaudhuri equation.

Proposition 7.1. Let $\mathscr{M}$ be a space-time that obeys the null energy condition, $\operatorname{Ric}(X, X)=R_{\alpha \beta} X^{\alpha} X^{\beta} \geq 0$ for all null vectors $X$, and let $\mathscr{N}$ be a smooth null hypersurface in $\mathscr{M}$. If the null generators of $\mathscr{N}$ are future geodesically complete, then the null generators of $\mathscr{N}$ have nonnegative expansion, $\theta \geq 0$. 
Proof. Suppose $\theta<0$ at $p \in \mathscr{N}$. Let $\eta:[0, \infty) \rightarrow \mathscr{N}, s \rightarrow \eta(s)$, be the null geodesic generator of $\mathscr{N}$ passing through $p=\eta(0)$. By rescaling $K$ if necessary, we can assume that $\eta$ is affinely parameterized. Let $\theta=\theta(s), s \in[0, \infty)$, be the null expansion of $\mathscr{N}$ along $\eta$; hence $\theta(0)<0$. Raychaudhuri's equation and the null energy condition imply that $\theta=\theta(s)$ obeys the inequality

$$
\frac{d \theta}{d s} \leq-\frac{1}{n-1} \theta^{2}
$$

and hence $\theta<0$ for all $s>0$. Dividing through by $\theta^{2}$ then gives

$$
\frac{d}{d s}\left(\frac{1}{\theta}\right) \geq \frac{1}{n-1},
$$

which implies that $1 / \theta \rightarrow 0$, i.e., $\theta \rightarrow-\infty$ in finite affine parameter time, contradicting the smoothness of $\theta$.

We wish to indicate the connection of Proposition 7.1 with the theory of black holes. In fact, this proposition is the most rudimentary form of Hawking's famous area theorem [154. Let $\mathscr{M}$ be a standard black hole space-time as defined, for example, in [154. It is not necessary to go into the technical details of the definition. It suffices to say that in $\mathscr{M}$ there exists a region $\mathscr{B}$, the black hole region, from which signals (future-directed causal curves) cannot escape to infinity (recall the example of the Schwarzschild solution discussed in Section 3.1). The boundary of this region is the event horizon $\mathscr{E}$, which, in general, is a Lipschitz hypersurface ruled by future inextendible null geodesics, called its null generators. If $\mathscr{E}$ is smooth and if its generators are future-complete, then Proposition 7.1]implies that $\mathscr{E}$ has nonnegative null expansion. This in turn implies that cross-sections of $\mathscr{E}$ are nondecreasing in area as one moves towards the future, as asserted by the area theorem. In the context of black hole thermodynamics, the area theorem is referred to as the second law of black mechanics, and provides a link between gravity and quantum physics. As it turns out, the area theorem remains valid without imposing any smoothness assumptions; for a recent study of the area theorem, which focuses on these issues of regularity, see [103.

7.2. Trapped and marginally trapped surfaces. We begin with some definitions. Let $\Sigma=\Sigma^{n-1}, n \geq 3$, be a spacelike submanifold of codimension two in a space-time $\left(\mathscr{M}^{n+1}, g\right)$. Regardless of the dimension of space-time, we shall refer to $\Sigma$ as a surface, which it actually is in the $3+1$ case. We are primarily interested in the case where $\Sigma$ is compact (without boundary), and so we simply assume this from the outset.

Each normal space of $\Sigma,\left[T_{p} \Sigma\right]^{\perp}, p \in \Sigma$, is timelike and two dimensional, and hence, admits two future-directed null directions orthogonal to $\Sigma$. Thus, if the normal bundle is trivial, $\Sigma$ admits two smooth nonvanishing future-directed null normal vector fields $l_{+}$and $l_{-}$, which are unique up to positive pointwise scaling; see Figure 7.1] By convention, we refer to $l_{+}$as outward pointing and $l_{-}$as inward pointing 14 In relativity it is standard to decompose the second fundamental form of $\Sigma$ into two scalar-valued null second forms $\chi_{+}$and $\chi_{-}$, associated to $l_{+}$and $l_{-}$, respectively. For each $p \in \Sigma, \chi_{ \pm}: T_{p} \Sigma \times T_{p} \Sigma \rightarrow \mathbb{R}$ is the bilinear form defined by

$$
\chi_{ \pm}(X, Y)=g\left(\nabla_{X} l_{ \pm}, Y\right) \text { for all } X, Y \in T_{p} \Sigma .
$$

\footnotetext{
${ }^{14}$ In many situations, there is a natural choice of inward and outward.
} 


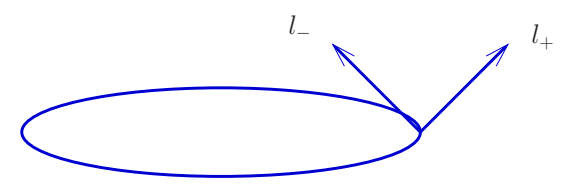

FiguRE 7.1. The null future normals $l_{ \pm}$to $\Sigma$.

A standard argument shows that $\chi_{ \pm}$is symmetric. Hence, $\chi_{+}$and $\chi_{-}$can be traced with respect to the induced metric $\gamma$ on $\Sigma$ to obtain the null mean curvatures (or null expansion scalars)

$$
\theta_{ \pm}=\operatorname{tr}_{\gamma} \chi_{ \pm}=\gamma^{i j}\left(\chi_{ \pm}\right)_{i j}=\operatorname{div}_{\Sigma} l_{ \pm}
$$

$\theta_{ \pm}$depends on the scaling of $l_{ \pm}$in a simple way. As follows from equation (7.5), multiplying $l_{ \pm}$by a positive function $f$ simply scales $\theta_{ \pm}$by the same function. Thus, the sign of $\theta_{ \pm}$does not depend on the scaling of $l_{ \pm}$. Physically, $\theta_{+}$(resp., $\theta_{-}$) measures the divergence of the outgoing (resp., ingoing) light rays emanating from $\Sigma$.

It is useful to note the connection between the null expansion scalars $\theta_{ \pm}$and the expansion of the generators of a null hypersurface, as discussed in Section 7.1. Let $\mathscr{N}_{+}$be the null hypersurface, defined and smooth near $\Sigma$, generated by the null geodesics passing through $\Sigma$ with initial tangents $l_{+}$. Then $\theta_{+}$is the null expansion of $\mathscr{N}_{+}$restricted to $\Sigma$. $\theta_{-}$may be described similarly.

Let $M$ be a spacelike hypersurface in a space-time $(\mathscr{M}, g)$ with induced metric $h$ and second fundamental form $K$, and suppose $\Sigma$ is embedded as a two-sided hypersurface in $M$. Then the null expansions $\theta_{ \pm}$can be expressed in terms of the initial data $(M, h, K)$ as follows. Since $\Sigma$ is two-sided in $M$, it admits a smooth unit normal field $\nu$ in $M$, unique up to sign. By convention, we refer to such a choice as outward pointing. Letting $u$ denote the future-directed unit normal to $M, l_{+}=u+\nu$ (resp., $l_{-}=u-\nu$ ) is a future-directed outward (resp., future-directed inward) pointing null normal vector field along $\Sigma$. Let $\theta_{ \pm}$be the null expansion with respect to the null normal $l_{ \pm}$. Then

$$
\theta_{ \pm}=\operatorname{tr}_{\Sigma} K \pm H
$$

where $\operatorname{tr}_{\Sigma} K$ is the trace of the projection of $K$ into $\Sigma$ with respect to the induced metric on $\Sigma$, and $H$ is the mean curvature of $\Sigma$ in $M$.

For round spheres in Euclidean slices of Minkowski space, with the obvious choice of inside and outside, one has $\theta_{-}<0$ and $\theta_{+}>0$. In fact, this is the case in general for large radial spheres in asymptotically flat spacelike hypersurfaces. However, in regions of space-time where the gravitational field is strong, one may have both $\theta_{-}<0$ and $\theta_{+}<0$, in which case $\Sigma$ is called a trapped surface. For example, the black hole region, $0<r<2 m$, in $(n+1)$-dimensional Schwarzschild spacetime (see Section 13.1) is foliated by spherically symmetric $(n-1)$-spheres, all of which are trapped surfaces. Under appropriate energy and causality conditions, the occurrence of a trapped surface signals the onset of gravitational collapse. This is the implication of the Penrose singularity theorem [238, the first of the famous singularity theorems. 
Theorem 7.2 (Penrose 238). Let $\mathscr{M}$ be a globally hyperbolic space-time with noncompact Cauchy surfaces satisfying the null energy condition. If $\mathscr{M}$ contains a trapped surface $\Sigma$, then $\mathscr{M}$ is future null geodesically incomplete.

Recall from Section 7.1 that the null energy condition is the curvature requirement, $\operatorname{Ric}(X, X)=R_{\alpha \beta} X^{\alpha} X^{\beta} \geq 0$ for all null vectors $X$. If a space-time $\mathscr{M}$ obeys the Einstein equation (2.8), then one can express the null energy condition in terms of the energy momentum tensor: $\mathscr{M}$ obeys the null energy condition if and only if $\mathcal{T}(X, X)=T_{i j} X^{i} X^{j} \geq 0$ for all null vectors $X$.

In studying an isolated gravitating system, such as the gravitational collapse of a star, it is customary to model the situation by a space-time which is asymptotically flat. In this context the assumption of Theorem 7.2 that the space-time admits a noncompact Cauchy surface is natural. The conclusion in the theorem of future null geodesic incompleteness is an indication that space-time "comes to an end" or develops a singularity somewhere in the causal future. However, the theorem gives no information about the nature of the singularity.

The existence of vacuum asymptotically flat initial data sets, with one asymptotic region and containing compact trapped surfaces, has been established by Beig and Ó Murchadha 34.

An intriguing question that arises in this context is whether a trapped surface can develop dynamically from initial data that did not contain any. This has been addressed by Christodoulou in [87, where a formidable analysis of the focusing effect of sufficiently strong incoming gravitational waves is presented. Some further developments can be found in [191.

To continue our discussion, consider again the general setting of a spacelike surface $\Sigma^{n-1}$ in a space-time $\mathscr{M}^{n+1}$ with future-directed null normal fields $l_{ \pm}$and associated null expansion scalars $\theta_{ \pm}$. Focusing attention on just the outward null normal $l_{+}$, we say that $\Sigma$ is an outer trapped surface (resp., weakly outer trapped surface) if $\theta_{+}<0$ (resp., $\theta_{+} \leq 0$ ). If $\theta_{+}$vanishes, we say that $\Sigma$ is a marginally outer trapped surface, or MOTS for short. In what follows we will be primarily concerned with properties of MOTSs.

MOTSs arise naturally in a number of situations. As an outgrowth of their work on the positive energy theorem, Schoen and Yau 261] showed that suitable conditions on the energy density and momentum density of an asymptotically flat initial data set ensure the presence of a MOTS; see also [286. Next, as follows from our comments about the area theorem in Section 7.1. cross-sections of the event horizon in black hole space-times have nonnegative expansion $\theta \geq 0$. (By a cross-section, we mean a smooth intersection of the event horizon with a spacelike hypersurface.) In the steady state limit this expansion goes to zero. Thus, it is a basic fact that cross-sections of the event horizon in stationary black hole spacetimes are MOTSs. For dynamical black hole space-times, MOTSs typically occur in the black hole region, i.e., the region inside the event horizon. While there are heuristic arguments for the existence of MOTSs in this situation, based on looking at the boundary of the trapped region [154, 277] within a given spacelike slice, using an approach put forth by Schoen [254, their existence has been rigorously established under natural physical conditions, first by Andersson and Metzger [10] in dimension three, and then by Eichmair 131] up to dimension seven; see Section 7.5 .

As noted earlier, MOTSs may be viewed as space-time analogues of minimal surfaces in Riemannian geometry. In fact, as follows from equation (7.7), in the 
time-symmetric case $(K=0)$ a MOTS is simply a minimal surface in $M$. Of importance for certain applications is the fact, first discussed by Andersson, Mars and Simon [7, 8], that MOTSs admit a notion of stability analogous to that for minimal surfaces.

7.3. Stability of MOTSs. In Riemannian geometry, a minimal surface (surface with vanishing mean curvature) is stable provided, for a suitable class of variations, the second variation of area is nonnegative, $\delta^{2} A \geq 0$. Stability of minimal surfaces can also be characterized in terms of the associated stability operator. This latter approach extends to MOTSs, as we now describe.

Let $\Sigma$ be a MOTS in $M$ with outward unit normal $\nu$. Consider a normal variation of $\Sigma$ in $M$, i.e., a map $F:(-\epsilon, \epsilon) \times \Sigma \rightarrow M$, such that (i) $F(0, \cdot)=$ id $_{\Sigma}$ and (ii) $\left.\frac{\partial F}{\partial t}\right|_{t=0}=\phi \nu, \phi \in C^{\infty}(\Sigma)$. Let $\theta(t)$ denote the null expansion of $\Sigma_{t}:=F(t, \Sigma)$ with respect to $l_{t}=u+\nu_{t}$, where $u$ is the future-directed timelike unit normal to $M$ and $\nu_{t}$ is the outer unit normal to $\Sigma_{t}$ in $M$. A computation shows

$$
\left.\frac{\partial \theta}{\partial t}\right|_{t=0}=L(\phi)
$$

where $L: C^{\infty}(\Sigma) \rightarrow C^{\infty}(\Sigma)$ is the operator [8, 145]

$$
L(\phi)=-\triangle \phi+2\langle X, \nabla \phi\rangle+\left(\frac{1}{2} S-(\rho+J(\nu))-\frac{1}{2}|\chi|^{2}+\operatorname{div} X-|X|^{2}\right) \phi .
$$

In the above, $\triangle, \nabla$, and div are the Laplacian, gradient and divergence operator, respectively, on $\Sigma, S$ is the scalar curvature of $\Sigma, \rho$ and $J$ are the energy density and momentum density, respectively, as defined in equations (5.3), (5.4), $X$ is the vector field on $\Sigma$ defined by taking the tangential part of $\nabla_{\nu} u$ along $\Sigma$, and $\langle$, denotes the induced metric on $\Sigma$.

In the time-symmetric case, $\theta$ in (7.8) becomes the mean curvature $H$, the vector field $X$ vanishes, and $L$ reduces to the classical stability operator (linearization of the mean curvature operator) of minimal surface theory. In analogy with the minimal surface case, we refer to $L$ in (7.9) as the stability operator associated with variations in the null expansion $\theta$. Although in general $L$ is not self-adjoint, its principal eigenvalue (eigenvalue with smallest real part) $\lambda_{1}(L)$ is real. Moreover, there exists an associated eigenfunction $\phi$ which is positive on $\Sigma$. Continuing the analogy with the minimal surface case, we say that a MOTS is stable provided $\lambda_{1}(L) \geq 0$. (In the minimal surface case this is equivalent to the second variation of area being nonnegative.) It follows from basic properties of $L$ that a MOTS $\Sigma$ is stable if and only if there exists a normal variation of $\Sigma$, with $\phi>0$, such that $\left.\frac{\partial \theta}{\partial t}\right|_{t=0} \geq 0$.

Stable MOTSs arise naturally in physical situations, for example, as outermost MOTSs. We say that $\Sigma$ is an outermost MOTS in $M$ provided there are no weakly outer trapped $\left(\theta_{+} \leq 0\right)$ surfaces outside of, and homologous to, $\Sigma$. We say that $\Sigma$ is a weakly outermost MOTS in $M$ provided there are no outer trapped $\left(\theta_{+}<0\right)$ surfaces outside of, and homologous to, $\Sigma$. Clearly, "outermost" implies "weakly outermost". Moreover we have the following [7, 8]:

Proposition 7.3. Weakly outermost MOTSs are stable.

To see this, consider a variation $\left\{\Sigma_{t}\right\}$ of a weakly outermost MOTS $\Sigma$ with variation vector field $\mathcal{V}=\phi \nu$, where $\phi$ is a positive eigenfunction associated to the principal eigenvalue $\lambda_{1}=\lambda_{1}(L)$. If $\lambda_{1}<0$, then equation (7.8) implies $\left.\frac{\partial \theta}{\partial t}\right|_{t=0}=$ 
$\lambda_{1} \phi<0$. Since $\theta(0)=0$, this implies that, for small $t>0, \Sigma_{t}$ is outer trapped, contrary to $\Sigma$ being weakly outermost.

A standard fact in the theory of black holes is that, for black hole space-times obeying the null energy condition, there can be no weakly outer trapped surfaces contained in the domain of outer communications (the region outside of all black holes and white holes). It follows that compact cross-sections of the event horizon in stationary black hole space-times obeying the null energy condition are stable MOTSs. Moreover, results of Andersson and Metzger [9, 10, provide natural criteria for the existence of outermost MOTSs in initial data sets containing trapped regions; see Section 7.5 .

Stable MOTSs share a number of properties in common with stable minimal surfaces. This sometimes depends on the following fact. Consider the symmetrized operator $L_{0}: C^{\infty}(\Sigma) \rightarrow C^{\infty}(\Sigma)$,

$$
L_{0}(\phi)=-\triangle \phi+\left(\frac{1}{2} S-(\rho+J(\nu))-\frac{1}{2}|\chi|^{2}\right) \phi,
$$

formally obtained by setting $X=0$ in (7.9). The key argument in [145] shows the following (see also [8, 144]).

Proposition 7.4. $\lambda_{1}\left(L_{0}\right) \geq \lambda_{1}(L)$.

In the next subsection we consider an application of stable MOTS to the topology of black holes.

7.4. On the topology of black holes. A useful step in the proof of black hole uniqueness (see Section 3.7) is Hawking's theorem on the topology of black holes 154, which asserts that compact cross-sections of the event horizon in $(3+1)$ dimensional, appropriately regular, asymptotically flat stationary black hole spacetimes obeying the dominant energy condition are topologically two-spheres. As shown by Hawking [153, this conclusion also holds for outermost MOTSs in spacetimes that are not necessarily stationary. The proof in both cases is variational in nature and relies on the classical Gauss-Bonnet theorem. Developments in physics related to string theory have led to an increased interest in the study of gravity, and in particular black holes, in higher dimensions; see, e.g., 134 for a recent review. The remarkable example of Emparan and Reall [133] of a $4+1$ asymptotically flat stationary vacuum black space-time with horizon topology $S^{1} \times S^{2}$, the socalled black ring, shows that horizon topology need not be spherical in higher dimensions. This example naturally led to the question of what are the allowable horizon topologies in higher-dimensional black hole space-times. This question was addressed in the papers of 144, 145, resulting in a natural generalization of Hawking's topology theorem to higher dimensions, which we now discuss.

Consider a space-time $\left(\mathscr{M}^{n+1}, g\right), n \geq 3$, satisfying the Einstein equations (not necessarily vacuum), and for simplicity assume that the cosmological constant vanishes, $\Lambda=0$. Let $M$ be a spacelike hypersurface in $\mathscr{M}$, which gives rise to the initial data set $(M, h, K)$, as in Section 7.3 . Recall from equation (5.15) that the dominant energy condition holds with respect to this initial data set provided $\rho \geq|J|$ along $M$, where $\rho$ and $J$ are the energy density and momentum density, respectively, as defined in Section 5.1 (but with $\Lambda$ set to zero).

The following result, obtained in 145, gives a natural extension of Hawking's black hole topology theorem to higher dimensions. Recall that a Riemannian manifold $\Sigma$ is of positive Yamabe type if it admits a metric of positive scalar curvature. 
Theorem 7.5. Let $\Sigma^{n-1}$ be a stable MOTS in an initial data set $\left(M^{n}, h, K\right)$, $n \geq 3$.

(1) If $\rho>|J|$ along $\Sigma$, then $\Sigma$ is of positive Yamabe type.

(2) If $\rho \geq|J|$ along $\Sigma$, then $\Sigma$ is of positive Yamabe type unless $\Sigma$ is Ricci flat (flat if $n=2,3), \chi=0$ and $\rho+J(\nu)=0$ along $\Sigma$.

In the time-symmetric case, Theorem 7.5 reduces to the classical result of Schoen and Yau 258, critical to their study of manifolds of positive scalar curvature, that a compact stable minimal hypersurface in a Riemannian manifold of positive scalar curvature is of positive Yamabe type.

The key to the proof of Theorem 7.5 is Proposition 7.4, which, since $\Sigma$ is assumed stable, implies that $\lambda_{1}\left(L_{0}\right) \geq 0$, where $L_{0}$ is the operator given in (7.10). Now, in effect, the proof has been reduced to the Riemannian case. Consider $\Sigma$ in the conformally related metric, $\tilde{\gamma}=\phi^{\frac{2}{n-2}} \gamma$, where $\gamma$ is the induced metric on $\Sigma$ and $\phi$ is a positive eigenfunction corresponding to $\lambda_{1}\left(L_{0}\right)$. The scalar curvatures $\tilde{S}$ and $S$ of the metrics $\tilde{\gamma}$ and $\gamma$, respectively, are related by (compare (5.11))

$$
\begin{aligned}
\tilde{S} & =\phi^{-\frac{n}{n-2}}\left(-2 \triangle \phi+S \phi+\frac{n-1}{n-2} \frac{|\nabla \phi|^{2}}{\phi}\right) \\
& =\phi^{-\frac{2}{n-2}}\left(2 \lambda_{1}\left(L_{0}\right)+2(\rho+J(\nu))+|\chi|^{2}+\frac{n-1}{n-2} \frac{|\nabla \phi|^{2}}{\phi^{2}}\right),
\end{aligned}
$$

where for the second equation we have used (7.10). Since $\rho+J(\nu) \geq \rho-|J| \geq 0$, we have that $\tilde{S} \geq 0$. By further standard metric deformations, the scalar curvature of $\Sigma$ can be made strictly positive, unless various quantities vanish identically.

According to Theorem[7.5] apart from certain exceptional circumstances, a stable marginally outer trapped surface $\Sigma$ in a space-time $\mathscr{M}$ obeying the dominant energy condition is of positive Yamabe type. Assume for the following discussion that $\Sigma$ is orientable. Then, in the standard case: $\operatorname{dim} \mathscr{M}=3+1$ (and hence $\operatorname{dim} \Sigma=2$ ), Gauss-Bonnet tells us that if $\Sigma$ is positive Yamabe, then $\Sigma$ is topologically a twosphere, and we recover Hawking's theorem. In higher dimensions, much is now known about topological obstructions to the existence of metrics of positive scalar curvature. While the first major result along these lines is the famous theorem of Lichnerowicz [198] concerning the vanishing of the $\hat{A}$ genus, a key advance in our understanding was made in the late 1970s and early 1980s by Schoen and Yau [256, 258, and Gromov and Lawson [147, 148. Let us focus on the case: $\operatorname{dim} \mathscr{M}=4+1$, and hence $\operatorname{dim} \Sigma=3$. Then by results of Schoen-Yau and Gromov-Lawson, in light of the resolution of the Poincaré conjecture, $\Sigma$ must be diffeomorphic to a finite connected sum of spherical spaces (spaces which are covered by the three-sphere) and $S^{2} \times S^{1}$ 's. Indeed, by the prime decomposition theorem, $\Sigma$ can be expressed as a connected sum of spherical spaces, $S^{2} \times S^{1}$ 's, and $K(\pi, 1)$ manifolds (manifolds whose universal covers are contractible). But as $\Sigma$ admits a metric of positive scalar curvature, it cannot have any $K(\pi, 1)$ 's in its prime decomposition. Hence, the basic horizon topologies in $\operatorname{dim} \mathscr{M}=4+1$ are $S^{3}$ and $S^{2} \times S^{1}$ (in the sense that $\Sigma$ is "built up" from such spaces), both of which are realized by nontrivial black hole space-times. It remains an interesting open question which topologies of positive Yamabe type can be realized as outermost MOTSs; see 264 for examples involving products of spheres.

A drawback of Theorem [7.5] is that it allows certain possibilities that one would like to rule out. For example, the theorem does not rule out the possibility of a 
vacuum black hole space-time with toroidal horizon topology (this borderline case also arises in the proof of Hawking's theorem). In fact, one can construct examples of stable toroidal MOTSs in space-times obeying the dominant energy condition. Such MOTSs cannot, however, be outermost, as the following theorem asserts.

Theorem 7.6 (144). Let $\Sigma$ be an outermost MOTS in the spacelike hypersurface $M$ and assume the dominant energy condition (3.15) holds in a space-time neighborhood of $\Sigma 15$ Then $\Sigma$ is of positive Yamabe type.

As an immediate corollary, we have that compact cross-sections of event horizons in regular stationary black hole space-times obeying the dominant energy condition are of positive Yamabe type. In particular, there can be no toroidal horizons.

Theorem 7.6 is an immediate consequence of the following rigidity result.

Theorem 7.7 ([144]). Let $\Sigma$ be a weakly outermost MOTS in the spacelike hypersurface $M$ and assume the dominant energy condition holds in a space-time neighborhood of $\Sigma$. If $\Sigma$ is not of positive Yamabe type, then there exists an outer neighborhood $U \approx[0, \epsilon) \times \Sigma$ of $\Sigma$ in $M$ such that each slice $\Sigma_{t}=\{t\} \times \Sigma, t \in[0, \epsilon)$ is a MOTS.

Theorem 7.7 is proved in two stages. The first stage, and the main effort, is to establish Theorem 7.7 subject to the additional assumption that $M$ has nonpositive mean curvature, $\tau \leq 0$. This is a purely initial data result, and the proof is carried out in two steps. The first step involves an inverse function theorem argument to show that an outer neighborhood of $\Sigma$ can be foliated by surfaces $\Sigma_{t}$ of constant null expansion, $\theta(t)=c_{t}$. This uses the stability of $\Sigma$ in a critical way. In the second step it is shown that all of these constants are zero, $c_{t}=0$. It is here where the sign of the mean curvature of $M$ needs to be controlled. Once having proved Theorem 7.7. subject to the condition, $\tau \leq 0$, the next stage, which is actually easy, is a deformation argument (specifically, a deformation of $M$ near $\Sigma$ ) that reduces the problem to the case $\tau \leq 0$.

We remark that Riemannian versions of Theorem 7.7 had previously been considered in 61, 62.

7.5. Existence of MOTSs. As mentioned earlier, compact cross-sections of the event horizon in regular stationary black hole space-times are necessarily MOTSs. In dynamical black hole space-times, it is typical for trapped or outer trapped surfaces to form in the black hole region. But the occurrence of an outer trapped surface in a spacelike hypersurface that obeys a mild asymptotic flatness condition leads to the existence of a MOTS. This follows from the existence result alluded to near the end of Section 7.2, and which we now discuss [10, 131, 254].

Let $\Omega$ be a relatively compact domain in a spacelike hypersurface $M$ with smooth boundary $\partial \Omega$. We assume that $\partial \Omega$ decomposes as a disjoint union of components, $\partial \Omega=\Sigma_{\text {inn }} \cup \Sigma_{\text {out }}$, where we think of $\Sigma_{\text {inn }}$ as the inner boundary and $\Sigma_{\text {out }}$ as the outer boundary of $\Omega$ in $M$. We choose the normal along $\Sigma_{\text {inn }}$ that points into $\Omega$, and the normal along $\Sigma_{\text {out }}$ that points out of $\Omega$, so that both normals point towards the region exterior to $\Sigma_{\text {out }}$. Thus, $\Sigma_{\text {inn }}$ is outer trapped if $\theta<0$ with respect to the future-directed null normal field along $\Sigma_{\text {inn }}$ that projects into $\Omega$. We say that $\Sigma_{\text {out }}$

\footnotetext{
${ }^{15}$ Note that since we are assuming $\Lambda=0$ here, the dominant energy condition is equivalent to the condition, $G_{\rho \nu} X^{\rho} Y^{\nu} \geq 0$ for all future-directed causal vectors $X, Y$, where $G_{\mu \nu}$ is the Einstein tensor.
} 
is outer untrapped if $\theta>0$ with respect to the future-directed null normal along $\Sigma_{\text {out }}$ that projects out of $\Omega$. Heuristically, if $\Sigma_{\text {out }}$ is lying in a region where $M$ is flattening out, then we expect $\Sigma_{\text {out }}$ to be outer untrapped.

With this notation and terminology we have the following existence result for MOTS.

Theorem 7.8 (10, 131). Let $M^{n}$ be a spacelike hypersurface in a space-time $\left(\mathscr{M}^{n+1}, g\right)$, with $n \leq 7$. Let $\Omega$ be a relatively compact domain in $M$, with smooth boundary $\partial \Omega=\Sigma_{\text {inn }} \cup \Sigma_{\text {out }}$, such that the inner boundary $\Sigma_{\text {inn }}$ is outer trapped and the outer boundary $\Sigma_{\text {out }}$ is outer untrapped, as described above. Then there exists a smooth compact MOTS in $\Omega$ homologous to $\Sigma_{\mathrm{inn}}$.

Moreover, it has been shown that the MOTS constructed in Theorem 7.8 is stable $([10,5])$.

In the time-symmetric case, Theorem 7.8 reduces to a well-known existence result for stable minimal surfaces. In this case the barrier conditions in Theorem 7.8 simply say that $\Omega$ is a mean convex domain. One can then minimize area in the homology class of a surface in $\Omega$ parallel to $\Sigma_{\text {inn }}$ and apply standard compactness and regularity results of geometric measure theory [137] to obtain a smooth (provided $\operatorname{dim} M \leq 7$ ) stable minimal surface in $\Omega$ homologous to $\Sigma_{\text {inn }}$. However, since MOTSs do not arise as stationary points of some elliptic functional, such a procedure does not work for general initial data. A completely different approach must be taken.

In fact, the proof of Theorem 7.8 is based on Jang's equation [180, which is closely related to the MOTS condition $\theta^{+}=0$. Given an initial data set $(M, g, K)$, consider the Riemannian product manifold, $\hat{M}=\mathbb{R} \times M, \hat{g}=d t^{2}+g$, and extend $K$ to $\hat{M}$ by taking it to be constant along the $t$-lines. Given a function $f$ on $M$, consider its graph, $N_{f}=\operatorname{graph} f=\{(t, x) \in \hat{M}: t=f(x), x \in M\}$, equipped with the induced metric. Then Jang's equation is the equation

$$
(H+P)(f)=H(f)+P(f)=0,
$$

where $H(f)=$ the mean curvature of $N_{f}$ and $P=\operatorname{tr}_{N_{f}} K$ (compare with equation (17.7) $)$.

Schoen and Yau 260] established existence and regularity for Jang's equation with respect to asymptotically flat initial sets as part of their approach to proving the positive mass theorem for general, nonmaximal, initial data sets. In the process they discovered an obstruction to global existence: solutions to Jang's equation tend to blow up in the presence of MOTS in the initial data $(M, g, K)$. This problematic blow-up behavior that Schoen and Yau had to contend with has now been turned on its head to become a feature of Jang's equation: in order to establish the existence of MOTS, one induces a blowup of the Jang equation. This is the approach taken in 10, 131 in a somewhat different situation.

In order to obtain solutions to Jang's equation, one considers the regularized equation

$$
(H+P)\left(f_{t}\right)=t f_{t} .
$$

In [131, Eichmair uses a Perron method to obtain solutions $f_{t}$ to (7.12) for $t$ sufficiently small, with values that tend to infinity in a small collared neighborhood of $\Sigma_{\text {out }}$, and that tend to minus infinity in a small collared neighborhood of $\Sigma_{\text {inn }}$, as $t \rightarrow 0$. The construction of Perron subsolutions and supersolutions makes use of 
the barrier conditions. Using the almost minimizing property [131 of the graphs $N_{t}:=\operatorname{graph} f_{t}$, one is able to pass to a smooth subsequential limit manifold $N$, bounded away from $\partial \Omega$, each component of which is either a cylinder or a graph that asymptotes to a cylinder. The projection into $M$ of such a cylinder produces the desired MOTS.

Under the barrier conditions of Theorem 7.8, Andersson and Metzger [10] were able to make use of this basic existence result to establish, for three-dimensional initial data sets, the existence of an outermost MOTS, thereby providing a rigorous proof of a long held "folk belief" in the theory of black holes that the boundary of the so-called outer trapped region in a time slice of space-time is a smooth MOTS. The key to proving the existence of an outermost MOTS is a compactness result for stable MOTS which follows from the extrinsic curvature estimates obtained by Andersson and Metzger in [9], together with an area bound obtained in [10. This area bound follows from an outer injectivity radius estimate for a certain class of MOTS, established by an interesting surgery procedure. The powerful methods developed by Eichmair in [130, 131 can be used to extend this result to $n$-dimensional initial data sets, $3 \leq n \leq 7$.

Consider, now, a foliation $\left\{M_{t}\right\}, a<t<b$, of a region of a space-time $\left(\mathscr{M}^{n+1}, g\right)$ by spacelike hypersurfaces $M_{t}$. If each slice $M_{t}$ admits an outermost MOTS $\Sigma_{t}$, then the family of MOTSs $\left\{\Sigma_{t}\right\}$ may form a hypersurface in space-time. A smooth spacelike hypersurface $H$ in a space-time $\left(\mathscr{M}^{n+1}, g\right)$ foliated by MOTS is called a marginally outer trapped tube (MOTT). In [7, 8], Andersson, Mars and Simon have obtained a rigorous existence result for MOTTs. Consider a spacelike foliation $\left\{M_{t}\right\}, a<t<b$, and suppose $\Sigma_{t_{0}}$ is a MOTS in $M_{t_{0}}$. They prove that if $\Sigma_{t_{0}}$ is strictly stable, i.e., if the principal eigenvalue of the associated stability operator (7.9) is strictly positive, then there exists a MOTT $H$ such that for $t$ close to $t_{0}$, $\Sigma_{t}:=H \cap M_{t}$ is a MOTS. For further related results see [6].

Many challenging questions concerning the global existence and behavior of MOTTs remain open. Building on the analysis of Dafermos [117, Williams [283] gave sufficient conditions on spherically symmetric black hole space-times satisfying the dominant energy condition, insuring that a spherically symmetric MOTT exists, is achronal, and is asymptotic to the event horizon. Understanding the generic asymptotic behavior of MOTTs in the nonspherically symmetric case remains an interesting open problem.

A MOTT satisfying certain supplementary conditions gives rise to the notion of a dynamical horizon, which provides an alternative, quasi-local description of a black hole. For an extensive review of dynamical horizons and related concepts, including physical applications, see [17.

\section{Appendix A. Open problems}

We compile here a list of interesting open problems discussed in the paper. They all appear to be difficult, of varying degrees of difficulty, with some most likely intractable in the foreseeable future.

(1) Remove the hypotheses of analyticity, nondegeneracy, and connectedness in the black hole uniqueness Theorem 3.2. p. 585.

(2) Construct a five-dimensional, stationary, $I^{+}$-regular vacuum black hole with two-dimensional group of isometries, or show that there are no such black holes. More generally, classify such black holes. Compare p. 586. 
(3) Prove that the positive energy theorem holds in all dimensions without the spin assumption. Compare p. 596.

(4) Find the optimal differentiability conditions for Theorem 4.1. More generally, construct a coherent local well-posedness theory for the evolution and constraint equations for metrics with low differentiability. Compare Remark 4.2, p. 588.

(5) Describe in a constructive way the set of solutions of the vacuum constraint equations on compact, asymptotically flat, and asymptotically hyperbolic manifolds, with arbitrary smooth initial data. Compare p. 594.

(6) Formulate, and prove, a precise version of Conjecture 6.9. p. 614. More generally, formulate and prove a precise version of the BKL conjecture, or find an open set of metrics developing a singularity which do not exhibit a BKL-type behavior.

(7) Show that generic Bianchi IX orbits have a dense $\omega$-limit set on the Kasner circle. Compare p. 612.

(8) Show that uniqueness and existence of maximal globally hyperbolic developments (Theorem 6.1, p. 602) holds in an optimal weak differentiability class. This requires revisiting the whole causality theory for metrics of low differentiability.

(9) Remove the condition of closed generators in Theorem 6.2, p. 602. More generally, show that existence of Cauchy horizons, not necessarily compact or analytic, implies existence of local isometries, or construct a counterexample.

(10) Generalize Ringström's Theorem 6.3. p. 603, to Gowdy models on $S^{2} \times S^{1}$, on $S^{3}$, and on lens spaces $L(p, q)$.

(11) Show that some twisting $U(1) \times U(1)$-symmetric vacuum models on $\mathbb{T}^{3}$ have mixmaster behavior. More generally, find an open set of such models with mixmaster behavior. Even better, analyze exhaustively the asymptotic behavior of those models; see Section 6.1.2 p. 604.

(12) Show that degenerate asymptotically flat spherically symmetric EinsteinMaxwell scalar field solutions are nongeneric. Compare Section6.1.3 p. 605. Analyze what happens in solutions in which trapped surfaces do not form. More generally, prove weak and strong cosmic censorship within this class of space-times.

(13) Find an open set of $U(1)$-symmetric metrics where the dynamics can be analyzed in the contracting direction. More generally, analyze exhaustively the dynamics of those models; see Section 6.3.1. p. 606.

(14) Show that polyhomogeneous initial data in Theorem 6.6. p. 609, lead to solutions with polyhomogeneous behavior at null infinity.

(15) Show that the Kerr solution is stable against small vacuum perturbations. Compare Section 6.5. p. 610.

(16) Determine what topologies of positive Yamabe type can be realized as outermost MOTSs in space-times satisfying the dominant energy condition. Compare p. 622.

(17) Prove initial data equivalents of Theorems 7.6 and 7.7. p. 623.

(18) Describe the generic behavior near the event horizon of marginally trapped tubes in black hole space-times; see p. 625 . 


\section{ACKNOWLEDGMENTS}

We are grateful to the Banff International Research Station and to the MittagLeffler Institute for hospitality at various stages of work on this project. The first and third authors acknowledge the friendly hospitality of the University of Miami during part of the work on this paper. We are indebted to Jim Isenberg and Catherine Williams for many useful comments on a previous version of the manuscript. Further thanks are due to Catherine Williams for providing some figures and for helping with others and to Woei-Chet Lim for providing Figure 6.2

\section{About The AUthors}

Piotr T. Chruściel is professor of gravitational physics at the University of Vienna. His research focuses on global problems arising in Einstein's theory of gravitation. Greg Galloway is professor of mathematics at the University of Miami. His primary research interests are in space-time geometry and mathematical relativity. Daniel Pollack is professor of mathematics at the University of Washington. His research areas include geometric analysis, partial differential equations, and general relativity.

\section{REFERENCES}

1. S. Alexakis, A.D. Ionescu, and S. Klainerman, Hawking's local rigidity theorem without analyticity, (2009), arXiv:0902.1173.

2. P. Allen, A. Clausen, and J. Isenberg, Near-constant mean curvature solutions of the Einstein constraint equations with non-negative Yamabe metrics, Class. Quantum Grav. 25 (2008), 075009, 15 pp., arxiv:0710.0725v1[gr-qc]. MR2404418 (2009a:83009)

3. L. Andersson, The global existence problem in general relativity, The Einstein Equations and the Large Scale Behavior of Gravitational Fields (P.T. Chruściel and H. Friedrich, eds.), Birkhäuser, Basel, 2004, arXiv:gr-qc/9911032v4, pp. 71-120. MR.2098914 (2005k:58054)

4. L. Andersson and P. Blue, Hidden symmetries and decay for the wave equation on the Kerr spacetime, (2009), arXiv:0908.2265 [math.AP].

5. L. Andersson, M. Eichmair, and J. Metzger, Jang's equation and its applications to marginally trapped surfaces (2010), arXiv:1006.4601.

6. L. Andersson, M. Mars, J. Metzger, and W. Simon, The time evolution of marginally trapped surfaces, Class. Quant. Grav. 26 (2009), 085018.

7. L. Andersson, M. Mars, and W. Simon, Local existence of dynamical and trapping horizons, Phys. Rev. Lett. 95 (2005), 111102.

8. _ Stability of marginally outer trapped surfaces and existence of marginally outer trapped tubes, Adv. Theor. Math. Phys. 12 (2008), 853-888. MR2420905 (2010a:83005)

9. L. Andersson and J. Metzger, Curvature estimates for stable marginally trapped surfaces, (2005), arXiv:gr-qc/0512106.

10. The area of horizons and the trapped region, arXiv:0708.4252 [gr-qc]. Comm. Math. Phys. 290 (2009), 941-972. MR2525646 (2010f:53118)

11. L. Andersson and V. Moncrief, Einstein spaces as attractors for the Einstein flow, in preparation.

12. — Elliptic-hyperbolic systems and the Einstein equations, Ann. Henri Poincaré 4 (2003), 1-34, arXiv:gr-qc/0110111. MR.1967177(2004c:58060)

13. _ Future complete vacuum space-times, The Einstein Equations and the Large Scale Behavior of Gravitational Fields (P.T. Chruściel and H. Friedrich, eds.), Birkhäuser, Basel, 2004, pp. 299-330, arXiv:gr-qc/0303045. MR2098919 (2006c:83004)

14. L. Andersson and A.D. Rendall, Quiescent cosmological singularities, Commun. Math. Phys. 218 (2001), 479-511, arXiv:gr-qc/0001047. MR2002h:83072

15. R. Arnowitt, S. Deser, and C.W. Misner, The dynamics of general relativity, Gravitation: An introduction to current research, Wiley, New York, 1962, pp. 227-265. MR0143629|(26:1182) 
16. A. Ashtekar and G.J. Galloway, Some uniqueness results for dynamical horizons, Adv. Theor. Math. Phys. 9 (2005), 1-30. MR2193368 (2006k:83101)

17. A. Ashtekar and B. Krishnan, Isolated and dynamical horizons and their applications, Living Rev. Rel. 7 (2004), gr-qc/0407042.

18. J.D. Barrow and F.J. Tipler, Analysis of the generic singularity studies by Belinskiน, Khalatnikov, and Lifschitz, Phys. Rep. 56 (1979), 371-402. MR555355 (83c:83005)

19. R. Bartnik, The existence of maximal hypersurfaces in asymptotically flat space-times, Commun. Math. Phys. 94 (1984), 155-175. MR761792 (86b:53063)

20. - The mass of an asymptotically flat manifold, Commun. Pure Appl. Math. 39 (1986), 661-693. MR 849427 (88b:58144)

21. _ Regularity of variational maximal surfaces, Acta Math. 161 (1988), 145-181. MR 971795 (90b:58255)

22. Remarks on cosmological spacetimes and constant mean curvature surfaces, Commun. Math. Phys. 117 (1988), 615-624. MR953823 (89g:53094)

23. S_ Some open problems in mathematical relativity, Conference on Mathematical Relativity (Canberra, 1988), Proc. Centre Math. Anal. Austral. Nat. Univ., vol. 19, Austral. Nat. Univ., Canberra, 1989, pp. 244-268. MR 1020805 (90g:83001)

24. __ Phase space for the Einstein equations, Commun. Anal. Geom. 13 (2005), 845-885, arXiv:gr-qc/0402070. MR2216143 (2007d:83012)

25. R. Bartnik, P.T. Chruściel, and N. Ó Murchadha, On maximal surfaces in asymptotically flat space-times., Commun. Math. Phys. 130 (1990), 95-109. MR1055687 (91b:53078)

26. R. Bartnik and J. Isenberg, The constraint equations, The Einstein equations and the large scale behavior of gravitational fields, Birkhäuser, Basel, 2004, pp. 1-38. MR2098912 (2005j:83007)

27. J.K. Beem, P.E. Ehrlich, and K.L. Easley, Global Lorentzian geometry, Second ed., Marcel Dekker Inc., New York, 1996. MR1384756 (97f:53100)

28. F. Béguin, Aperiodic oscillatory asymptotic behavior for some Bianchi spacetimes, (2010), arXiv:1004.2984 [gr-qc].

29. R. Beig, TT-tensors and conformally flat structures on 3-manifolds, Mathematics of Gravitation, Part I (Warsaw, 1996), Banach Center Publ., vol. 41, Polish Acad. Sci., Warsaw, 1997, pp. 109-118. MR1466511 (98k:53040)

30. R. Beig and P.T. Chruściel, Killing Initial Data, Class. Quantum. Grav. 14 (1997), A83A92, A special issue in honour of Andrzej Trautman on the occasion of his 64th Birthday, J.Tafel, editor. MR 1691888 (2000c:83011)

31. K_ Killing vectors in asymptotically flat space-times: I. Asymptotically translational Killing vectors and the rigid positive energy theorem, Jour. Math. Phys. 37 (1996), 1939 1961, arXiv:gr-qc/9510015. MR 1380882 (97d:83033)

32. - The asymptotics of stationary electro-vacuum metrics in odd space-time dimensions, Class. Quantum Grav. 24 (2007), 867-874. MR2297271

33. R. Beig, P.T. Chruściel, and R. Schoen, KIDs are non-generic, Ann. Henri Poincaré 6 (2005), 155-194, arXiv:gr-qc/0403042. MR2121280 (2005m:83013)

34. R. Beig and N. Ó Murchadha, Vacuum spacetimes with future trapped surfaces, Class. Quantum Grav. 13 (1996), 739-751. MR1383704(97e:83005)

35. V.A. Belinski, I.M. Khalatnikov, and E.M. Lifshtitz, Oscillatory approach to a singular point in the relativistic cosmology, Adv. Phys. 19 (1970), 525-573.

36. B. Berger, P.T. Chruściel, J. Isenberg, and V. Moncrief, Global foliations of vacuum spacetimes with $T^{2}$ isometry, Ann. Phys. (NY) 260 (1997), 117-148. MR1474313 (98j:83005)

37. B. Berger, J. Isenberg, and M. Weaver, Oscillatory approach to the singularity in vacuum space-times with $T^{2}$ isometry, Phys. Rev. D64 (2001), 084006, arXiv:gr-qc/0104048, erratum-ibid. D67, 129901 (2003).

38. B.K. Berger, Hunting local mixmaster dynamics in spatially inhomogeneous cosmologies, Class. Quantum Grav. 21 (2004), S81-S95, A space-time safari: Essays in honour of Vincent Moncrief. MR 2053000 (2005f:83066)

39. B.K. Berger, D. Garfinkle, J. Isenberg, V. Moncrief, and M. Weaver, The singularity in generic gravitational collapse is spacelike, local, and oscillatory, Mod. Phys. Lett. A13 (1998), 1565-1574, arXiv:gr-qc/9805063. MR1633408 (99j:83052)

40. B.K. Berger and V. Moncrief, Exact $U(1)$ symmetric cosmologies with local Mixmaster dynamics, Phys. Rev. D62 (2000), 023509, arXiv:gr-qc/0001083. MR1790740 (2001f:83027) 
41. Signature for local mixmaster dynamics in U(1) symmetric cosmologies, Phys. Rev. D 62 (2000), 123501, 9, arXiv:gr-qc/0006071. MR1813870 (2001k:83027)

42. A.N. Bernal and M. Sánchez, Smoothness of time functions and the metric splitting of globally hyperbolic space-times, Commun. Math. Phys. 257 (2005), 43-50. MR2163568 (2006g:53105)

43. A.L. Besse, Einstein manifolds, Ergebnisse der Mathematik und ihrer Grenzgebiete. 3. Folge, vol. 10, Springer Verlag, Berlin, New York, Heidelberg, 1987. MR867684 (88f:53087)

44. L. Bieri and N. Zipser, Extensions of the stability theorem of the Minkowski space in general relativity. AMS/IP Studies in Advanced Mathematics, vol. 45, Amer. Math. Soc., Providence, RI, 2009. MR2531716

45. G. D. Birkhoff, Relativity and modern physics, Harvard University Press, 1923

46. P. Blue, Decay of the Maxwell field on the Schwarzschild manifold, arXiv:0710.4102 [math.AP]. J. Hyperbolic Differ. Equ. 5 (2008), 807-856. MR2475482 (2010d:35371)

47. P. Blue and J. Sterbenz, Uniform decay of local energy and the semi-linear wave equation on Schwarzchild space, arXiv:math.AP/0510315. Comm. Math. Phys. 268 (2006), 481-504. MR:2259204 (2007i:58037)

48. H.L. Bray, Proof of the Riemannian Penrose inequality using the positive mass theorem, Jour. Diff. Geom. 59 (2001), 177-267. MR1908823 (2004j:53046)

49. _ Black holes and the Penrose inequality in general relativity, Proceedings of the International Congress of Mathematicians, Vol. II (Beijing, 2002), Higher Ed. Press, 2002, pp. 257-271. MR.1957038(2004b:83056)

50. _ Black holes, geometric flows, and the Penrose inequality in general relativity, Notices Amer. Math. Soc. 49 (2002), 1372-1381. MR1936643 (2003j:83052)

51. H.L. Bray and P.T. Chruściel, The Penrose inequality, The Einstein Equations and the Large Scale Behavior of Gravitational Fields (P.T. Chruściel and H. Friedrich, eds.), Birkhäuser, Basel, 2004, pp. 39-70, arXiv:gr-qc/0312047. MR2098913 (2005m:83014)

52. H.L. Bray and M.A. Khuri, A Jang Equation Approach to the Penrose Inequality, (2009), arXiv:0910.4785 [math.DG].

53. , PDE's which imply the Penrose conjecture, (2009), arXiv:0905.2622 [math.DG].

54. H.L. Bray and D.A. Lee, On the Riemannian Penrose inequality in dimensions less than eight, Duke Math. Jour. 148 (2009), 81-106, arXiv:0705.1128. MR2515101

55. H.L. Bray and P. Miao, On the capacity of surfaces in manifolds with nonnegative scalar curvature, Invent. Math. 172 (2008), 459-475. MR.2393076

56. H.L. Bray and R. Schoen, Recent proofs of the Riemannian Penrose conjecture, Current Developments in Mathematics, 1999 (Cambridge, MA), Int. Press, Somerville, MA, 1999, pp. 1-36. MR1990246(2004j:53047)

57. Hubert Bray, Sean Hayward, Marc Mars, and Walter Simon, Generalized inverse mean curvature flows in spacetime, Commun. Math. Phys. 272 (2007), 119-138, arXiv:gr-qc/0603014. MR.2291804 (2008c:53065)

58. P. Breitenlohner, D. Maison, and G. Gibbons, 4-dimensional black holes from Kaluza-Klein theories, Commun. Math. Phys. 120 (1988), 295-333. MR973537 (89j:83018)

59. R. Brout, S. Massar, R. Parentani, and P. Spindel, A primer for black hole quantum physics, Phys. Rept. 260 (1995), 329-454. MR1353182 (97g:83064)

60. R. Budic, J. Isenberg, L. Lindblom, and P. Yasskin, On the determination of the Cauchy surfaces from intrinsic properties, Commun. Math. Phys. 61 (1978), 87-95. MR 0489695 (58:9089)

61. M. Cai, Volume minimizing hypersurfaces in manifolds of nonnegative scalar curvature, Minimal Surfaces, Geometric Analysis and Symplectic Geometry (Baltimore, MD, 1999), Adv. Stud. Pure Math., vol. 34, Math. Soc. Japan, Tokyo, 2002, pp. 1-7. MR1925731 (2003f:53104)

62. M. Cai and G.J. Galloway, Rigidity of area minimizing tori in 3-manifolds of nonnegative scalar curvature, Commun. Anal. Geom. 8 (2000), 565-573. MR:1775139 (2001j:53051)

63. E. Calabi, On Ricci curvature and geodesics, Duke Math. J. 34 (1967), 667-676. MR0216429 $(35: 7262)$

64. A. Carrasco and M. Mars, A counter-example to a recent version of the Penrose conjecture, (2009), arXiv:0911.0883 [gr-qc].

65. B. Carter, Global structure of the Kerr family of gravitational fields, Phys. Rev. 174 (1968), 1559-1571. 
66. _ Black hole equilibrium states, Black Holes (C. de Witt and B. de Witt, eds.), Gordon \& Breach, New York, London, Paris, 1973, Proceedings of the Les Houches Summer School. MR 0465047 (57:4960)

67. M. Chae and P.T. Chruściel, On the dynamics of Gowdy space times, Commun. Pure Appl. Math. 57 (2004), 1015-1074, arXiv:gr-qc/0305029. MR/2053758 (2005i:83085)

68. Y. Choquet-Bruhat, Positive-energy theorems, Relativity, groups and topology, II (Les Houches, 1983) (B.S. deWitt and R. Stora, eds.), North-Holland, Amsterdam, 1984, pp. 739785. MR $830248(87 \mathrm{~g}: 83054)$

69. _ Einstein constraints on compact $n$-dimensional manifolds, Class. Quantum Grav. 21 (2004), S127-S151, arXiv:gr-qc/0311029. MR2053003 (2005g:83011)

70. _ Future complete $U(1)$ symmetric Einsteinian space-times, the unpolarized case, The Einstein Equations and the Large Scale Behavior of Gravitational Fields (P.T. Chruściel and H. Friedrich, eds.), Birkhäuser, Basel, 2004, pp. 251-298. MR2098918 (2006b:83015)

71. _ General relativity and the Einstein equations, Oxford University Press, Oxford, UK, 2009. MR2473363

72. Y. Choquet-Bruhat and D. Christodoulou, Elliptic systems in $H_{s, \delta}$ spaces on manifolds which are Euclidean at infinity, Acta Math. 146 (1981), 129-150. MR594629 (82c:58060)

73. Y. Choquet-Bruhat, P.T. Chruściel, and J. Loizelet, Global solutions of the EinsteinMaxwell equations in higher dimension, Class. Quantum Grav. (2006), 7383-7394, arXiv:grqc/0608108. MR2279722(2008i:83022)

74. Y. Choquet-Bruhat, P.T. Chruściel, and J.M. Martín-García, The light-cone theorem, Class. Quantum Grav. 26 (2009), 135011 (22 pp.), arXiv:0905.2133 [gr-qc]. MR2515694

75. Y. Choquet-Bruhat and R. Geroch, Global aspects of the Cauchy problem in general relativity, Commun. Math. Phys. 14 (1969), 329-335. MR0250640(40:3872)

76. Y. Choquet-Bruhat, J. Isenberg, and V. Moncrief, Solutions of constraints for Einstein equations, C. R. Acad. Sci. Paris Sér. I Math. 315 (1992), 349-355. MR1179734(93h:58151)

77. Y. Choquet-Bruhat, J. Isenberg, and D. Pollack, The Einstein-scalar field constraints on asymptotically Euclidean manifolds, Chinese Ann. Math. Ser. B 27 (2006), 31-52, arXiv:grqc/0506101. MR2209950(2007h:58049)

78. - Applications of theorems of Jean Leray to the Einstein-scalar field equations, Jour. Fixed Point Theory Appl. 1 (2007), 31-46, arXiv:gr-qc/0611009. MR.2282342

79. The constraint equations for the Einstein-scalar field system on compact manifolds, Class. Quantum Grav. 24 (2007), 809-828, arXiv:gr-qc/0610045. MR 2297268

80. Y. Choquet-Bruhat and V. Moncrief, Future global in time Einsteinian space-times with U(1) isometry group., Ann. Henri Poincaré 2 (2001), 1007-1064. MR1877233(2003g:53123)

81. Y. Choquet-Bruhat and J. York, The Cauchy problem, General Relativity (A. Held, ed.), Plenum Press, New York, 1980, pp. 99-172. MR583716 (82k:58028)

82. D. Christodoulou, Global solutions of nonlinear hyperbolic equations for small initial data, Commun. Pure Appl. Math. 39 (1986), 267-282. MR820070 (87c:35111)

83. - The problem of a self-gravitating scalar field, Commun. Math. Phys. 105 (1986), 337-361. MR 848643 (87i:83009)

84. Examples of naked singularity formation in the gravitational collapse of a scalar field, Ann. of Math. (2) 140 (1994), 607-653. MR1307898(95j:83100)

85. - The instability of naked singularities in the gravitational collapse of a scalar field, Ann. of Math. (2) 149 (1999), 183-217. MR1680551(2000a:83086)

86. On the global initial value problem and the issue of singularities, Class. Quantum Grav. 16 (1999), A23-A35. MR.1728432 (2001a:83010)

87. _ The Formation of Black Holes in General Relativity, arXiv:0805.3880 [gr-qc]. European Mathematical Society, Zurich, 2009. MR2488976 (2009k:83010)

88. D. Christodoulou and S. Klainerman, The global nonlinear stability of the Minkowski space, Princeton University Press, 1993. MR.1316662 (95k:83006)

89. D. Christodoulou and N. Ó Murchadha, The boost problem in general relativity, Commun. Math. Phys. 80 (1981), 271-300. MR623161(84e:83011)

90. D. Christodoulou and A. Shadi Tahvildar-Zadeh, On the asymptotic behavior of spherically symmetric wave maps, Duke Math. Jour. 71 (1993), 31-69. MR1230285 (94j:58044)

91. _ On the regularity of spherically symmetric wave maps, Commun. Pure Appl. Math. 46 (1993), 1041-1091. MR.1223662 (94e:58030) 
92. P.T. Chruściel, Boundary conditions at spatial infinity from a Hamiltonian point of view, Topological Properties and Global Structure of Space-Time (P. Bergmann and V. de Sabbata, eds.), Plenum Press, New York, 1986, pp. 49-59, URL http://www.phys.univ-tours. fr/ ${ }^{\sim}$ piotr/scans.

93. , On space-times with $\mathrm{U}(1) \times \mathrm{U}(1)$ symmetric compact Cauchy surfaces, Ann. Phys. 202 (1990), 100-150. MR1067565 (91h:83007)

94. __ On uniqueness in the large of solutions of Einstein equations ("strong cosmic censorship”), Contemp. Math. 132 (1992), 235-273. MR1188443 (93g:83078)

95. - On completeness of orbits of Killing vector fields, Class. Quantum Grav. 10 (1993), 2091-2101, arXiv:gr-qc/9304029. MR/1242398 (94i:53063)

96. _ Beijing lecture notes on mathematical relativity, www.phys.univ-tours.fr/ piotr/ papers/BeijingAll.pdf 2006.

97. P.T. Chruściel and J. Cortier, On the geometry of Emparan-Reall black rings, (2008), arXiv:0807.2309 [gr-qc].

98. P.T. Chruściel, J. Cortier, and A. Garcia-Parrado, On the global structure of the PomeranskySenkov black holes, (2009), arXiv:0911.0802 [gr-qc].

99. P.T. Chruściel and J. Lopes Costa, On uniqueness of stationary black holes, Astérisque No. 321 (2008), 195-265, arXiv:0806.0016v2 [gr-qc]. MR2521649

100. P.T. Chruściel and E. Delay, Existence of non-trivial, vacuum, asymptotically simple spacetimes, Class. Quantum Grav. 19 (2002), L71-L79, arXiv:gr-qc/0203053, erratum-ibid, 3389. MR.1902228 (2003e:83024a); MR1920322 (2003e:83024b)

101. On mapping properties of the general relativistic constraints operator in weighted function spaces, with applications, Mém. Soc. Math. de France. 94 (2003), vi+103, arXiv:grqc/0301073v2. MR2031583(2005f:83008)

102. _ Manifold structures for sets of solutions of the general relativistic constraint equations, Jour. Geom. Phys. 51 (2004), 442-472, arXiv:gr-qc/0309001v2. MR2085346 (2005i:83008)

103. P.T. Chruściel, E. Delay, G. Galloway, and R. Howard, Regularity of horizons and the area theorem, Ann. Henri Poincaré 2 (2001), 109-178, arXiv:gr-qc/0001003. MR 1823836 (2002e:83045)

104. P.T. Chruściel, J. Isenberg, and V. Moncrief, Strong cosmic censorship in polarised Gowdy space-times, Class. Quantum Grav. 7 (1990), 1671-1680. MR.1075858(91i:83042)

105. P.T. Chruściel, J. Isenberg, and D. Pollack, Gluing initial data sets for general relativity, Phys. Rev. Lett. 93 (2004), 081101, arXiv:gr-qc/0409047.

106. _ Initial data engineering, Commun. Math. Phys. 257 (2005), 29-42, arXiv:grqc/0403066. MR2163567(2007d:83013)

107. P.T. Chruściel and K. Lake, Cauchy horizons in Gowdy space times, Class. Quantum Grav. 21 (2004), S153-S170, arXiv:gr-qc/0307088. MR2053004 (2005d:83092)

108. P.T. Chruściel and D. Maerten, Killing vectors in asymptotically flat space-times: II. Asymptotically translational Killing vectors and the rigid positive energy theorem in higher dimensions, Jour. Math. Phys. 47 (2006), 022502, 10 pp., arXiv:gr-qc/0512042. MR.2208148 (2007b:83054)

109. P.T. Chruściel and L. Nguyen, A uniqueness theorem for degenerate Kerr-Newman black holes, (2010), arXiv:1002.1737 [gr-qc].

110. P.T. Chruściel, H.S. Reall, and K.P. Tod, On non-existence of static vacuum black holes with degenerate components of the event horizon, Class. Quantum Grav. 23 (2006), 549554, arXiv:gr-qc/0512041. MR2196372(2007b:83090)

111. P.T. Chruściel and A. Rendall, Strong cosmic censorship in vacuum space-times with compact, locally homogeneous Cauchy surfaces, Ann. Physics 242 (1995), 349-385. MR1349391 (96f:83085)

112. N.J. Cornish and J.J. Levin, The mixmaster universe: A chaotic Farey tale, Phys. Rev. D55 (1997), 7489-7510, arXiv:gr-qc/9612066.

113. J. Corvino, Scalar curvature deformation and a gluing construction for the Einstein constraint equations, Commun. Math. Phys. 214 (2000), 137-189. MR1794269(2002b:53050)

114. - On the existence and stability of the Penrose compactification, Ann. Henri Poincaré 8 (2007), 597-620. MR2329363 (2008e:53050)

115. J. Corvino and R.M. Schoen, On the asymptotics for the vacuum Einstein constraint equations, Jour. Diff. Geom. 73 (2006), 185-217, arXiv:gr-qc/0301071. MR2225517|(2007e:58044) 
116. M. Dafermos, Stability and instability of the Cauchy horizon for the spherically symmetric Einstein-Maxwell-scalar field equations, Ann. of Math. (2) 158 (2003), 875-928. MR2031855 (2005f:83009)

117. - The interior of charged black holes and the problem of uniqueness in general relativity, Commun. Pure Appl. Math. 58 (2005), 445-504, arXiv:gr-qc/0307013. MR 2119866 (2006e:83087)

118. M. Dafermos and I. Rodnianski, A proof of Price's law for the collapse of a self-gravitating scalar field, Invent. Math. 162 (2005), 381-457, arXiv:gr-qc/0309115. MR2199010 (2006i:83016)

119. A proof of the uniform boundedness of solutions to the wave equation on slowly rotating Kerr backgrounds, (2008), arXiv:0805.4309 [gr-qc].

120. , Lectures on black holes and linear waves, (2008), arXiv:0811.0354 [gr-qc].

121. - The red-shift effect and radiation decay on black hole spacetimes, Commun. Pure Appl. Math. 62 (2009), 859-919. MR2527808

122. S. Dain, Trapped surfaces as boundaries for the constraint equations, Class. Quantum Grav. 21 (2004), 555-574, Corrigendum ib. 22 (2005), p. 769; arXiv:gr-qc/0308009. MR 2030884 (2004m:83060); MR2118199 (2005j:83076)

123. S. Dain and H. Friedrich, Asymptotically flat initial data with prescribed regularity at infinity, Commun. Math. Phys. 222 (2001), no. 3, 569-609. MR.1888089 (2003f:58057)

124. T. Damour, M. Henneaux, and H. Nicolai, Cosmological billiards, Class. Quantum Grav. 20 (2003), R145-R200. MR1981434 (2004e:83124)

125. T. Damour, M. Henneaux, A. D. Rendall, and M. Weaver, Kasner-like behaviour for subcritical Einstein-matter systems, Ann. Henri Poincaré 3 (2002), 1049-1111. MR 1957378 (2004g:83092)

126. J. Demaret, J.-L. Hanquin, M. Henneaux, and P. Spindel, Nonoscillatory behaviour in vacuum Kaluza-Klein cosmologies, Phys. Lett. B 164 (1985), 27-30. MR815631 (87e:83060)

127. S.K. Donaldson, An application of gauge theory to four-dimensional topology, Jour. Diff. Geom. 18 (1983), 279-315. MR710056 (85c:57015)

128. R. Donninger, W. Schlag, and A. Soffer, A proof of Price's Law on Schwarzschild black hole manifolds for all angular momenta, (2009), arXiv:0908.4292 [gr-qc].

129. D. Eardley, E. Liang, and R. Sachs, Velocity-dominated singularities in irrotational dust cosmologies, Jour. Math. Phys. 13 (1972), 99-106.

130. M. Eichmair, Existence, regularity, and properties of generalized apparent horizons, arXiv:0805.4454 [math.DG]. Comm. Math. Phys. 294 (2010), 745-760. MR2585986

131. __ The plateau problem for apparent horizons, 2007, arXiv:0711.4139 [math.DG].

132. R. Emparan, Rotating circular strings, and infinite non-uniqueness of black rings, J. High Energy Phys. 03 (2004), 064. MR2061590 (2004m:83090)

133. R. Emparan and H.S. Reall, A rotating black ring in five dimensions, Phys. Rev. Lett. 88 (2002), 101101, arXiv:hep-th/0110260. MR 1901280(2003e:83060)

134. _ Black rings, Class. Quantum Grav. 23 (2006), R169-R197, arXiv:hep-th/0608012. $\operatorname{MR} 2270099(2008 \mathrm{a}: 83100)$

135. _ B B B _ Lack holes in higher dimensions, Living Rev. Rel. 11 (2008), 6, arXiv:0801.3471 [hep-th].

136. L.C. Evans, Partial differential equations, Graduate Studies in Mathematics, vol. 19, American Mathematical Society, Providence, RI, 1998. MR.1625845 (99e:35001)

137. H. Federer, Geometric measure theory, Springer-Verlag, New York, 1969 (Die Grundlehren der mathematischen Wissenschaften, Vol. 153). MR0257325(41:1976)

138. F. Finster, N. Kamran, J. Smoller, and S.-T. Yau, Linear waves in the Kerr geometry: A mathematical voyage to black hole physics, Bull. Amer. Math. Soc. (N.S.) 46 (2009), 635-659, arXiv:0801.1423 [math-ph]. MR2525736

139. A.E. Fischer and J.E. Marsden, The initial value problem and the dynamical formulation of general relativity, Einstein Centenary Volume (Hawking and Israel, eds.), Cambridge University Press, Cambridge, 1979, pp. 138-211.

140. Y. Fourès-Bruhat, Théorème d'existence pour certains systèmes d'équations aux dérivées partielles non linéaires, Acta Math. 88 (1952), 141-225. MR0053338 (14:756g)

141. J. Frauendiener, On the Penrose inequality, Phys. Rev. Lett. 87 (2001), 101101, 4 pp., arXiv:gr-qc/0105093. MR1854297 (2002f:83054) 
142. C. Fronsdal, Completion and embedding of the Schwarzschild solution, Phys. Rev. (2) 116 (1959), 778-781. MR0110524(22:1402)

143. G.J. Galloway, Some results on Cauchy surface criteria in Lorentzian geometry, Illinois Jour. Math. 29 (1985), 1-10. MR769754 (86c:53043)

144. _ Rigidity of marginally trapped surfaces and the topology of black holes, Commun. Anal. Geom. 16 (2008), 217-229, gr-qc/0608118. MR2411473(2009e:53087)

145. G.J. Galloway and R. Schoen, A generalization of Hawking's black hole topology theorem to higher dimensions, Comm. Math. Phys. 266 (2005), 571-576, arXiv:gr-qc/0509107. MR 2238889(2007i:53078)

146. R. Geroch, Domain of dependence, Jour. Math. Phys. 11 (1970), 437-449. MR0270697 $(42: 5585)$

147. M. Gromov and H.B. Lawson, Jr., Spin and scalar curvature in the presence of a fundamental group. I, Ann. of Math. (2) 111 (1980), 209-230. MR.569070 (81g:53022)

148. _ Positive scalar curvature and the Dirac operator on complete Riemannian manifolds, Inst. Hautes Études Sci. Publ. Math. (1983), 83-196. MR720933 (85g:58082)

149. T. Harmark, Stationary and axisymmetric solutions of higher-dimensional general relativity, Phys. Rev. D (3) 70 (2004), 124002, 25, arXiv:hep-th/0408141. MR2124693 (2005k:83136)

150. T. Harmark and P. Olesen, Structure of stationary and axisymmetric metrics, Phys. Rev. D (3) 72 (2005), 124017, 12, hep-th/0408141. MR2198031 (2007i:83042)

151. S. W. Hawking and R. Penrose, The singularities of gravitational collapse and cosmology, Proc. Roy. Soc. London Ser. A 314 (1970), 529-548. MR0264959 (41:9548)

152. S.W. Hawking, Black holes in general relativity, Commun. Math. Phys. 25 (1972), 152-166. MR0293962 (45:3037)

153. _ The event horizon, Black holes/Les astres occlus (B.S. DeWitt, ed.), Gordon and Breach Science Publishers, 1973, pp. xii+552+176. MR0408678 (53:12441)

154. S.W. Hawking and G.F.R. Ellis, The large scale structure of space-time, Cambridge University Press, Cambridge, 1973, Cambridge Monographs on Mathematical Physics, No. 1. MR0424186 (54:12154)

155. E. Hebey, F. Pacard, and D. Pollack, A variational analysis of Einstein-scalar field Lichnerowicz equations on compact Riemannian manifolds, Commun. Math. Phys. 278 (2008), no. 1, 117-132, arXiv:gr-qc/0702031. MR2367200

156. J.M. Heinzle, C. Uggla, and N. Röhr, The cosmological billiard attractor, arXiv:grqc/0702141. Adv. Theor. Math. Phys. 13 (2009), 293-407. MR2481269

157. M. Herzlich, A Penrose-like inequality for the mass of Riemannian asymptotically flat manifolds, Commun. Math. Phys. 188 (1997), 121-133. MR1471334(99a:53039)

158. M. Heusler, Black hole uniqueness theorems, Cambridge University Press, Cambridge, 1996. MR:1446003 (98b:83057)

159. C.G. Hewitt, J.T. Horwood, and J. Wainwright, Asymptotic dynamics of the exceptional Bianchi cosmologies, Class. Quantum Grav. 20 (2003), 1743-1756. MR.1981447 (2004g:83027)

160. S. Hollands, A. Ishibashi, and R.M. Wald, A higher dimensional stationary rotating black hole must be axisymmetric, Commun. Math. Phys. 271 (2007), 699-722, arXiv:grqc/0605106. MR2291793(2008c:83031)

161. S. Hollands and S. Yazadjiev, A Uniqueness theorem for 5-dimensional Einstein-Maxwell black holes, Class. Quantum Grav. 25 (2008), 095010, arXiv:0711.1722 [gr-qc]. MR 2417776 (2009c:83061)

162. , A uniqueness theorem for stationary Kaluza-Klein black holes, (2008), arXiv:0812.3036 [gr-qc]

163. _ Uniqueness theorem for 5-dimensional black holes with two axial Killing fields, Commun. Math. Phys. 283 (2008), 749-768, arXiv:0707.2775 [gr-qc]. MR2434746

164. M. Holst, G. Nagy, and G. Tsogtgerel, Rough solutions of the Einstein constraints on closed manifolds without near-CMC conditions, Commun. Math. Phys. 288 (2009), 547613. MR 2500992

165. _ Far-from-constant mean curvature solutions of Einstein's constraint equations with positive Yamabe metrics, Phys. Rev. Lett. 100 (2008), 161101, 4 pp. MR2403263 (2009c:53112) 
166. L. Hörmander, On the fully nonlinear Cauchy problem with small data. II, Microlocal analysis and nonlinear waves (Minneapolis, MN, 1988-1989), IMA Vol. Math. Appl., vol. 30, Springer, New York, 1991, pp. 51-81. MR1120284 (94c:35127)

167. G. Huisken and T. Ilmanen, The inverse mean curvature flow and the Riemannian Penrose inequality, Jour. Diff. Geom. 59 (2001), 353-437. MR.1916951(2003h:53091)

168. A.D. Ionescu and S. Klainerman, On the uniqueness of smooth, stationary black holes in vacuum, arXiv:0711.0040 [gr-qc]. Invent. Math. 175 (2009), 35-102. MR2461426 (2009j:83053)

169. J. Isenberg, Constant mean curvature solutions of the Einstein constraint equations on closed manifolds, Class. Quantum Grav. 12 (1995), 2249-2274. MR.1353772 (97a:83013)

170. J. Isenberg, D. Maxwell, and D. Pollack, Gluing of non-vacuum solutions of the Einstein constraint equations, Adv. Theor. Math. Phys. 9 (2005), 129-172. MR2193370|(2006j:83004)

171. J. Isenberg, R. Mazzeo, and D. Pollack, Gluing and wormholes for the Einstein constraint equations, Commun. Math. Phys. 231 (2002), 529-568, arXiv:gr-qc/0109045. MR 1946448 (2004a:83006)

172. On the topology of vacuum spacetimes, Ann. Henri Poincaré 4 (2003), 369-383. MR:1985777(2004h:53053)

173. J. Isenberg and V. Moncrief, Symmetries of cosmological Cauchy horizons with exceptional orbits, Jour. Math. Phys. 26 (1985), 1024-1027. MR787349 (86k:83023)

174. _ Some results on nonconstant mean curvature solutions of the Einstein constraint equations, Physics on Manifolds (M. Flato, R. Kerner, and A. Lichnerowicz, eds.). Mathematical Physics Studies, 15, Kluwer Academic Publishers, Dordrecht, 1994, Y. Choquet-Bruhat Festschrift, pp. 295-302. MR 1267081 (95h:58133)

175. _ A set of nonconstant mean curvature solutions of the Einstein constraint equations on closed manifolds, Class. Quantum Gravity 13 (1996), 1819-1847. MR.1400943 (97h:83010)

176. __ Asymptotic behavior of polarized and half-polarized $U(1)$ symmetric vacuum spacetimes, Class. Quantum Grav. 19 (2002), 5361-5386, arXiv:gr-qc/0203042. MR/1939922 (2003h:83035)

177. , Symmetries of higher dimensional black holes, Class. Quantum Grav. 25 (2008), 195015, arXiv:0805.1451. MR2438980 (2009g:83081)

178. J. Isenberg and N. Ó Murchadha, Non-CMC conformal data sets which do not produce solutions of the Einstein constraint equations, Class. Quantum Grav. 21 (2004), S233-S241, A space-time safari: Essays in honour of Vincent Moncrief; arXiv:gr-qc/0311057. MR 2053007 (2005c:83003)

179. J. Isenberg and M. Weaver, On the area of the symmetry orbits in $T^{2}$ symmetric spacetimes, Class. Quantum Grav. 20 (2003), 3783-3796, arXiv:gr-qc/0304019. MR2001695 (2004f:83049)

180. P. S. Jang, On the positivity of energy in general relativity, J. Math. Phys. 19 (1978), 11521155. MR 488515 (80b:83012a)

181. B.S. Kay and R.M. Wald, Theorems on the uniqueness and thermal properties of stationary, nonsingular, quasi-free states on space-times with a bifurcate horizon, Phys. Rep. 207 (1991), 49-136. MR1133130 (93b:81189)

182. S. Kichenassamy and A. Rendall, Analytic description of singularities in Gowdy space-times, Class. Quantum Grav. 15 (1998), 1339-1355. MR.1623091(99d:83077)

183. S. Klainerman, Uniform decay estimates and the Lorentz invariance of the classical wave equation, Commun. Pure Appl. Math. 38 (1985), 321-332. MR784477(86i:35091)

184. _ The null condition and global existence to nonlinear wave equations, Nonlinear Systems of Partial Differential Equations in Applied Mathematics, Part 1 (Santa Fe, N.M., 1984), Lectures in Appl. Math., vol. 23, Amer. Math. Soc., Providence, RI, 1986, pp. 293-326. MR837683 (87h:35217)

185. S. Klainerman and F. Nicolò, On local and global aspects of the Cauchy problem in general relativity, Class. Quantum Grav. 16 (1999), R73-R157. MR1709123 (2000h:83006)

186. - The evolution problem in general relativity, Progress in Mathematical Physics, vol. 25, Birkhäuser, Boston, MA, 2003. MR1946854 (2004f:58036)

187. _ Peeling properties of asymptotically flat solutions to the Einstein vacuum equations, Class. Quantum Grav. 20 (2003), 3215-3257. MR.1992002 (2005b:83038a)

188. S. Klainerman and I. Rodnianski, Ricci defects of microlocalized Einstein metrics, Jour. Hyperbolic Differ. Equ. 1 (2004), 85-113. MR2052472 (2005f:58048) 
189. - The causal structure of microlocalized rough Einstein metrics, Ann. of Math. (2) 161 (2005), 1195-1243. MR2180401(2007d:58052)

190. _ Rough solutions of the Einstein-vacuum equations, Ann. of Math. (2) 161 (2005), 1143-1193. MR2180400 (2007d:58051)

191. _ On emerging scarred surfaces for the Einstein vacuum equations, (2010), arXiv:1002.2656 [gr-qc].

192. M.D. Kruskal, Maximal extension of Schwarzschild metric, Phys. Rev. (2) 119 (1960), 17431745. MR0115757(22:6555)

193. J.M. Lee and T.H. Parker, The Yamabe problem, Bull. Amer. Math. Soc. (N.S.) 17 (1987), 37-91. MR888880 (88f:53001)

194. J. Leray, Hyperbolic differential equations, The Institute for Advanced Study, Princeton, NJ, 1953. MR0063548(16:139a)

195. T.-T. Li and Y.M Chen, Global classical solutions for nonlinear evolution equations, Pitman Monographs and Surveys in Pure and Applied Mathematics, vol. 45, Longman Scientific \& Technical, Harlow, 1992. MR.1172318 (93g:35002)

196. Y. Li and G. Tian, Nonexistence of axially symmetric, stationary solution of Einstein vacuum equation with disconnected symmetric event horizon, Manuscripta Math. 73 (1991), 83-89. MR1124312(92m:83022)

197. A. Lichnerowicz, L'intégration des équations de la gravitation relativiste et le problème des $n$ corps, Jour. Math. Pures Appl. (9) 23 (1944), 37-63. MR0014298 (7:266d)

198. A. Lichnerowicz, Spineurs harmoniques, C.R. Acad. Sci. Paris Sér. A-B 257 (1963), 7-9. MR0156292(27:6218)

199. S. Liebscher, J. Harterich, K. Webster, and M. Georgi, Ancient Dynamics in Bianchi Models: Approach to Periodic Cycles, (2010), arXiv:1004.1989 [gr-qc].

200. H. Lindblad and I. Rodnianski, The global stability of the Minkowski space-time in harmonic gauge, (2004), arXiv:math.ap/0411109.

201. Global existence for the Einstein vacuum equations in wave coordinates, Commun. Math. Phys. 256 (2005), 43-110, arXiv:math.ap/0312479. MR2134337(2006b:83020)

202. R.B. Lockhart, Fredholm properties of a class of elliptic operators on noncompact manifolds, Duke Math. J. 48 (1981), 289-312. MR610188 (82j:35050)

203. R.B. Lockhart and R.C. McOwen, On elliptic systems in $\mathbf{R}^{n}$, Acta Math. 150 (1983), 125135. MR $697610(84 \mathrm{~d}: 35048)$

204. _ Correction to: "On elliptic systems in $\mathbf{R}^{n "}$ [Acta Math. 150 (1983), no. 1-2, 125-135], Acta Math. 153 (1984), 303-304. MR.766267 (86a:35049)

205. __ Elliptic differential operators on noncompact manifolds, Ann. Scuola Norm. Sup. Pisa Cl. Sci. (4) 12 (1985), 409-447. MR837256 (87k:58266)

206. J. Loizelet, Solutions globales des équations d'Einstein-Maxwell en jauge harmonique et jauge de Lorenz, C. R. Acad. Sci. Sér. I 342 (2006), 479-482. MR2214599 (2007f:83026)

207. _ Problèmes globaux en relativité générale, Ph.D. thesis, Université de Tours, 2008, Www.phys.univ-tours.fr/ piotr/papers/TheseTitreComplet.pdf

208. E. Malec, Isoperimetric inequalities in the physics of black holes, Acta Phys. Polon. B 22 (1991), 829-858. MR 1151689 (93e:83046)

209. E. Malec, M. Mars, and W. Simon, On the Penrose inequality for general horizons, Phys. Rev. Lett. 88 (2002), 121102, arXiv:gr-qc/0201024. MR.1901724(2003d:83029)

210. J. Marzuola, J. Metcalfe, D. Tataru, and M. Tohaneanu, Strichartz estimates on Schwarzschild black hole backgrounds, arXiv:0802.3942v2 [math.AP]. Comm. Math. Phys. 293 (2010), 37-83. MR 2563798

211. D. Maxwell, A model problem for conformal parameterizations of the Einstein constraint equations, (2009), arXiv:0909.5674 [gr-qc].

212. _ A class of solutions of the vacuum Einstein constraint equations with freely specified mean curvature, arXiv:0804.0874v1 [gr-qc]. Math. Res. Lett. 16 (2009), 627-645. MR2525029

213. _ Rough solutions of the Einstein constraint equations on compact manifolds, Jour. Hyperbolic Diff. Equ. 2 (2005), 521-546, arXiv:gr-qc/0506085. MR2151120 (2006d:58027)

214. Solutions of the Einstein constraint equations with apparent horizon boundaries, Commun. Math. Phys. 253 (2005), 561-583, arXiv:gr-qc/0307117. MR 2116728 (2006c:83008)

215. R.C. McOwen, The behavior of the Laplacian on weighted Sobolev spaces, Commun. Pure Appl. Math. 32 (1979), 783-795. MR539158(81m:47069) 
216. Fredholm theory of partial differential equations on complete Riemannian manifolds, Pacific J. Math. 87 (1980), 169-185. MR590874 (82g:58082)

217. _ On elliptic operators in $\mathbf{R}^{n}$, Commun. Partial Differential Equations 5 (1980), 913933. MR $584101(81 \mathrm{k}: 35058)$

218. P. Miao, Y. Shi, and L.-F. Tam, On geometric problems related to Brown-York and Liu-Yau quasilocal mass, 2009, arXiv.org:0906.5451 [math.DG].

219. V. Moncrief, Spacetime symmetries and linearization stability of the Einstein equations. I, Jour. Mathematical Phys. 16 (1975), 493-498. MR0363398(50:15836)

220. _ Global properties of Gowdy spacetimes with $T^{3} \times \mathbb{R}$ topology, Ann. Phys. 132 (1981), 87-107. MR615961 (83e:83007)

221. _ Infinite-dimensional family of vacuum cosmological models with TaubNUT (Newman-Unti-Tamburino)-type extensions, Phys. Rev. D 23 (1981), 312-315. MR:82b:83024

222. V. Moncrief and D. Eardley, The global existence problem and cosmic censorship in general relativity, Gen. Rel. Grav. 13 (1981), 887-892. MR640355 (83b:83006)

223. V. Moncrief and J. Isenberg, Symmetries of cosmological Cauchy horizons, Commun. Math. Phys. 89 (1983), 387-413. MR/709474 (85c:83026)

224. Y. Morisawa and D. Ida, A boundary value problem for the five-dimensional stationary rotating black holes, Phys. Rev. D69 (2004), 124005, arXiv:gr-qc/0401100. MR2095953 (2005d:83079)

225. R.C. Myers and M.J. Perry, Black holes in higher dimensional space-times, Ann. Phys. 172 (1986), 304-347. MR868295 (88a:83074)

226. G. Neugebauer and J. Hennig, Non-existence of stationary two-black-hole configurations, Gen. Relativity Gravitation 41 (2009), no. 9, 2113-2130. MR2534657

227. G. Neugebauer and R. Meinel, Progress in relativistic gravitational theory using the inverse scattering method, Jour. Math. Phys. 44 (2003), 3407-3429, arXiv:gr-qc/0304086. MR $2006757(2004 \mathrm{k}: 83056)$

228. E. Newman, L. Tamburino, and T. Unti, Empty-space generalization of the Schwarzschild metric, Jour. Math. Phys. 4 (1963), 915-923. MR0152345 (27:2325)

229. J.-P. Nicolas, Dirac fields on asymptotically flat space-times, Dissertationes Math. (Rozprawy Mat.) 408 (2002), 1-85. MR1952742 (2004c:83068)

230. L. Nirenberg and H.F. Walker, The null spaces of elliptic partial differential operators in $\mathbf{R}^{n}$, J. Math. Anal. Appl. 42 (1973), 271-301, Collection of articles dedicated to Salomon Bochner. MR0320821 (47:9354)

231. N. Ó Murchadha and J.W. York, Jr., Existence and uniqueness of solutions of the Hamiltonian constraint of general relativity on compact manifolds, Jour. Mathematical Phys. 14 (1973), 1551-1557. MR0332094 (48:10421)

232. B. O'Neill, Semi-Riemannian geometry, Academic Press, New York, 1983. MR0719023 (85f:53002)

233. , The geometry of Kerr black holes, A.K. Peters, Wellesley, Mass., 1995. MR1328643 (96c:83052)

234. J. R. Oppenheimer and H. Snyder, On continued gravitational contraction, Phys. Rev. 56 (1939), 455-459.

235. F. Pacard and T. Rivière, Linear and nonlinear aspects of vortices, The Ginzburg-Landau model, Progress in Nonlinear Differential Equations and their Applications, 39, Birkhäuser Boston Inc., Boston, MA, 2000. MR1763040 (2001k:35066)

236. T. Parker and C.H. Taubes, On Witten's proof of the positive energy theorem, Commun. Math. Phys. 84 (1982), 223-238. MR661134(83m:83020)

237. R. Penrose, Asymptotic properties of fields and space-times, Phys. Rev. Lett. 10 (1963), 66-68. MR0149912 (26:7397)

238. _ Gravitational collapse and space-time singularities, Phys. Rev. Lett. 14 (1965), 57-59. MR.0172678(30:2897)

239. _ Gravitational collapse - the role of general relativity, Riv. del Nuovo Cim. (numero speziale) 1 (1969), 252-276.

240. F. Planchon and I. Rodnianski, On uniqueness for the Cauchy problem in general relativity, (2007), in preparation.

241. A.A. Pomeransky and R.A. Sen'kov, Black ring with two angular momenta, (2006), hepth/0612005. 
242. A.D. Rendall, Existence of constant mean curvature foliations in space-times with twodimensional local symmetry, Comm. Math. Phys. 189 (1997), 145-164, arXiv:gr-qc/9605022. MR:1478534 (99c:53075)

243. _ Global dynamics of the mixmaster model, Class. Quantum Grav. 14 (1997), 23412356. MR 1468587 (98j:83024)

244. _ Local and global existence theorems for the Einstein equations, Living Reviews in Relativity 1 (1998), URL http://www.livingreviews.org MR1711494 (2000g:58044)

245. __ Fuchsian analysis of singularities in Gowdy space-times beyond analyticity, Class. Quantum Grav. 17 (2000), 3305-3316, arXiv:gr-qc/0004044. MR1779512 (2002e:83066)

246. M. Reiterer and E. Trubowitz, The BKL Conjectures for Spatially Homogeneous Spacetimes, (2010), arXiv:1005.4908 [gr-qc]

247. A.D. Rendall and M. Weaver, Manufacture of Gowdy space-times with spikes, Class. Quantum Grav. 18 (2001), 2959-2975, arXiv:gr-qc/0103102. MR.1847897 (2002e:83010)

248. H. Ringström, Curvature blow up in Bianchi VIII and IX vacuum space-times, Class. Quantum Grav. 17 (2000), 713-731, arXiv:gr-qc/9911115. MR1744051 (2001d:83070)

249. _ The Bianchi IX attractor, Ann. Henri Poincaré 2 (2001), 405-500, arXiv:grqc/0006035. MR1846852 (2002h:83075)

250. _ Existence of an asymptotic velocity and implications for the asymptotic behavior in the direction of the singularity in $T^{3}$-Gowdy, Commun. Pure Appl. Math. 59 (2006), 977-1041. MR2222842

251. _ Strong cosmic censorship in $T^{3}$-Gowdy space-times, Ann. of Math. (2) 170 (2008), 1181-1240. MRMR2600872

252. D.C. Robinson, Uniqueness of the Kerr black hole, Phys. Rev. Lett. 34 (1975), 905-906.

253. R. Schoen, Variational theory for the total scalar curvature functional for Riemannian metrics and related topics, Topics in Calculus of Variations (Montecatini Terme, 1987), Lecture Notes in Math., vol. 1365, Springer, Berlin, 1989, pp. 120-154. MR994021 (90g:58023)

254. L Lecture at the Miami Waves Conference, January 2004.

255. _ Mean curvature in Riemannian geometry and general relativity, Global Theory of Minimal Surfaces, Clay Math. Proc., vol. 2, Amer. Math. Soc., Providence, RI, 2005, pp. 113-136. MR2167257(2006f:53044)

256. R. Schoen and S.-T. Yau, Existence of incompressible minimal surfaces and the topology of three-dimensional manifolds with nonnegative scalar curvature, Ann. of Math. (2) 110 (1979), 127-142. MR541332 (81k:58029)

257. On the proof of the positive mass conjecture in general relativity, Commun. Math. Phys. 65 (1979), 45-76. MR526976 (80j:83024)

258. _ On the structure of manifolds with positive scalar curvature, Manuscripta Math. 28 (1979), 159-183. MR535700 (80k:53064)

259. _ The energy and the linear momentum of space-times in general relativity, Commun. Math. Phys. 79 (1981), 47-51. MR609227 (82j:83045)

260. _ Proof of the positive mass theorem. II, Commun. Math. Phys. 79 (1981), 231-260. MR.612249 (83i:83045)

261. The existence of a black hole due to the condensation of matter, Commun. Math. Phys. 90 (1983), 575-579. MR719436 (84k:83005)

262. Conformally flat manifolds, Kleinian groups and scalar curvature, Invent. Math. 92 (1988), 47-71. MR931204 (89c:58139)

263. L__ Lectures on differential geometry, Conference Proceedings and Lecture Notes in Geometry and Topology, I, International Press, Cambridge, MA, 1994. MR 1333601(97d:53001)

264. F. Schwartz, Existence of outermost apparent horizons with product of spheres topology, arXiv:0704.2403 [gr-qc]. Comm. Anal. Geom. 16 (2008), 799-817. MR2471370 (2009m:53100)

265. H.J. Seifert, Smoothing and extending cosmic time functions, Gen. Rel. Grav. 8 (1977), 815-831. MR0484260 (58:4185)

266. Y. Shi and L.-F. Tam, Quasi-local mass and the existence of horizons, Comm. Math. Phys. 274 (2007), 277-295, arXiv:math.DG/0511398. MR2322904 (2008g:53095)

267. H.F. Smith and D. Tataru, Sharp local well-posedness results for the nonlinear wave equation, Ann. of Math. (2) 162 (2005), 291-366. MR2178963 (2006k:35193) 
268. D. Sudarsky and R.M. Wald, Extrema of mass, stationarity and staticity, and solutions to the Einstein-Yang-Mills equations, Phys. Rev. D46 (1993), 1453-1474. MR.1182084 (93k:83008)

269. L. Szabados, Quasi-local energy-momentum and angular momentum in GR: A review article, Living Rev. 4 (2004), URL http://relativity.livingreviews.org/Articles/lrr-2004-4

270. Gy. Szekeres, On the singularities of a Riemannian manifold, Gen. Rel. Grav. 34 (2002), 2001-2016, Reprinted from Publ. Math. Debrecen 7 (1960), 285-301 MR0125541|(23:A2842). MR 1945497 (2003j:83073a)

271. D. Tataru, Local decay of waves on asymptotically flat stationary space-times, (2008), arXiv:0910.5290 [math.AP].

272. D. Tataru and M. Tohaneanu, Local energy estimate on Kerr black hole backgrounds, (2008), arXiv:0810.5766 [math.AP].

273. A.H. Taub, Empty space-times admitting a three parameter group of motions, Ann. of Math. (2) 53 (1951), 472-490. MR0041565 (12:865b)

274. C.M. Taubes, Self-dual Yang-Mills connections on non-self-dual 4-manifolds, Jour. Diff. Geom. 17 (1982), 139-170. MR658473 (83i:53055)

275. C. Uggla, H. van Elst, J. Wainwright, and G.F.R. Ellis, The past attractor in inhomogeneous cosmology, Phys. Rev. D68 (2003), 103502 (22 pp.), arXiv:gr-qc/0304002.

276. J. Wainwright and L. Hsu, A dynamical systems approach to Bianchi cosmologies: Orthogonal models of class A, Class. Quantum Grav. 6 (1989), 1409-1431. MR1014971 (90h:83033)

277. R.M. Wald, General relativity, University of Chicago Press, Chicago, 1984. MR.757180 (86a:83001)

278. M.-T. Wang and S.-T. Yau, A generalization of Liu-Yau's quasi-local mass, Commun. Anal. Geom. 15 (2007), 249-282. MR 2344323 (2008h:53046)

279. - Isometric embeddings into the Minkowski space and new quasi-local mass, Commun. Math. Phys. 288 (2009), no. 3, 919-942. MR2504860 (2010d:53077)

280. _ Quasilocal mass in general relativity, Phys. Rev. Lett. 102 (2009), no. 2, no. 021101, 4 pp. MR2475769 (2010b:83015)

281. G. Weinstein, On rotating black-holes in equilibrium in general relativity, Commun. Pure Appl. Math. XLIII (1990), 903-948. MR1072397(91h:83063)

282. _ On the force between rotating coaxial black holes, Trans. Amer. Math. Soc. 343 (1994), 899-906. MR1214787(94h:83087)

283. C. Williams, Asymptotic behavior of spherically symmetric marginally trapped tubes, Ann. Henri Poincaré 9 (2008) 1029-1067. arXiv:gr-qc/0702101. MR2453255 (2009h:83095)

284. D.M. Witt, Vacuum space-times that admit no maximal slice, Phys. Rev. Lett. 57 (1986), 1386-1389. MR857257 (87h:83008)

285. E. Witten, A new proof of the positive energy theorem, Commun. Math. Phys. 80 (1981), 381-402. MR626707 (83e:83035)

286. S.-T. Yau, Geometry of three manifolds and existence of black hole due to boundary effect, Adv. Theor. Math. Phys. 5 (2001), 755-767. MR.1926294 (2003j:53052)

287. J.W. York, Jr., Covariant decompositions of symmetric tensors in the theory of gravitation, Ann. Inst. H. Poincaré Sect. A (N.S.) 21 (1974), 319-332. MR0373548 (51:9748)

LMPt, Fédération Denis Poisson, Tours; Mathematical Institute and Hertford ColLEGE, OXFORD

Current address: Hertford College, Oxford OX1 3BW, UK

E-mail address: chrusciel@maths.ox.ac.uk

Department of Mathematics, University of Miami

Current address: Coral Gables, Florida 33124

E-mail address: galloway@math.miami.edu

Department of Mathematics, University of Washington

Current address: Box 354350, Seattle, Washington 98195-4350, USA

E-mail address: pollack@math.washington.edu 